\title{
Identification of MltG as a Potential Terminase for Peptidoglycan Polymerization in Bacteria
}

\section{Citation}

Yunck, Rachel. 2016. Identification of MltG as a Potential Terminase for Peptidoglycan

Polymerization in Bacteria. Doctoral dissertation, Harvard University, Graduate School of Arts \& Sciences.

\section{Permanent link}

http://nrs.harvard.edu/urn-3:HUL.InstRepos:33493474

\section{Terms of Use}

This article was downloaded from Harvard University's DASH repository, and is made available under the terms and conditions applicable to Other Posted Material, as set forth at http:// nrs.harvard.edu/urn-3:HUL.InstRepos:dash.current.terms-of-use\#LAA

\section{Share Your Story}

The Harvard community has made this article openly available.

Please share how this access benefits you. Submit a story.

Accessibility 
Identification of MltG as a Potential Terminase for Peptidoglycan

Polymerization in Bacteria

A dissertation presented

by

Rachel Yunck

to

The Division of Medical Sciences

in partial fulfillment of the requirements

for the degree of

Doctor of Philosophy

in the subject of

Biological and Biomedical Sciences

Harvard University

Cambridge, Massachusetts

April 2016 
(C) 2016 Rachel Yunck

All rights reserved. 
Dissertation Advisor: Dr. Thomas G. Bernhardt

Rachel Yunck

\title{
Identification of MItG as a Potential Terminase for Peptidoglycan \\ Polymerization in Bacteria
}

\begin{abstract}
Bacterial cells are fortified against osmotic lysis by a cell wall made of peptidoglycan (PG). Synthases called penicillin-binding proteins (PBPs), the targets of penicillin and related antibiotics, polymerize the glycan strands of PG and crosslink them into the cell wall meshwork via attached peptides. The average length of glycan chains inserted into the matrix by the PBPs is thought to play an important role in bacterial morphogenesis, but polymerization termination factors controlling this process have yet to be discovered. Here, we report the identification of Escherichia coli $\mathrm{MltG}$ (YceG) as a potential terminase for glycan polymerization that is broadly conserved in bacteria. A clone containing $m l t G$ was initially isolated in a screen for multicopy plasmids generating a lethal phenotype in cells defective for the PG synthase PBP1b.

Biochemical studies revealed that MltG is an inner membrane enzyme with endolytic transglycosylase activity capable of cleaving at internal positions within a glycan polymer. Radiolabeling experiments further demonstrated MltG-dependent nascent PG processing in vivo, and bacterial two-hybrid analysis identified an MltG-PBP1b interaction. Mutants lacking MltG were also shown to have longer glycans in their PG relative to wild-type cells. Our combined results are thus consistent with a model in which MltG associates with PG synthetic complexes to cleave nascent polymers and terminate their elongation.
\end{abstract}




\section{Table of Contents}

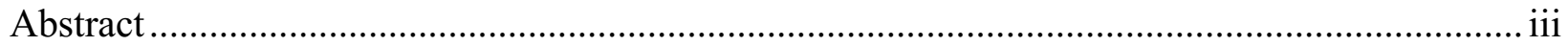

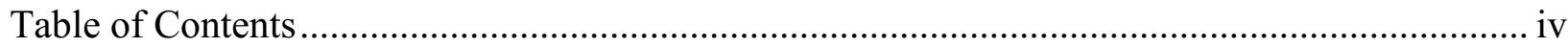

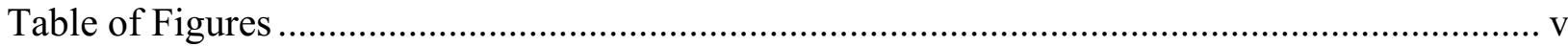

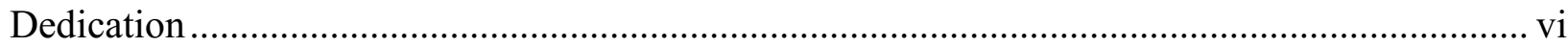

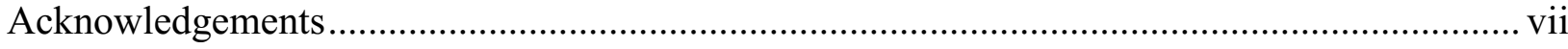

Chapter 1. Introduction to Lytic Transglycosylases in $E$. coli................................................... 1

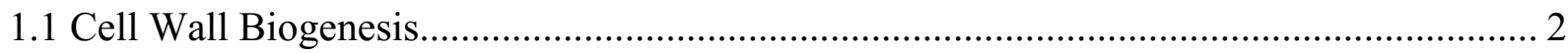

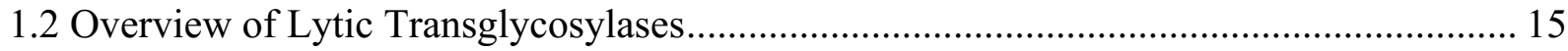

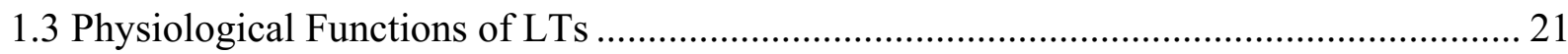

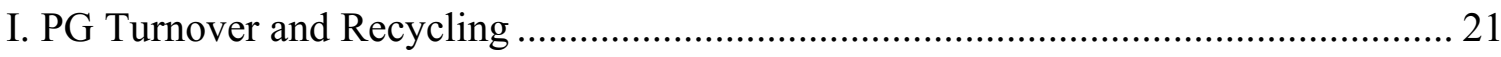

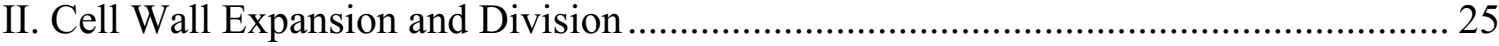

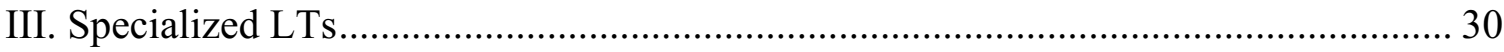

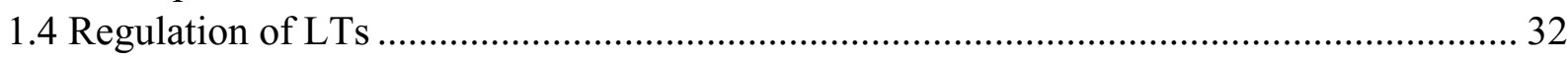

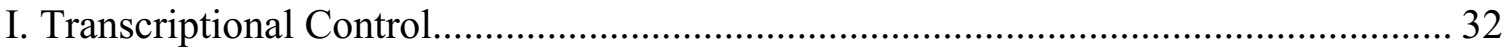

II. Post-translational Control........................................................................................ 33

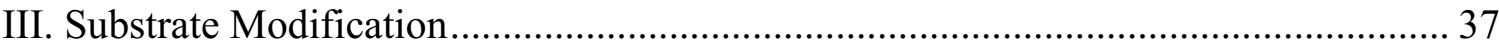

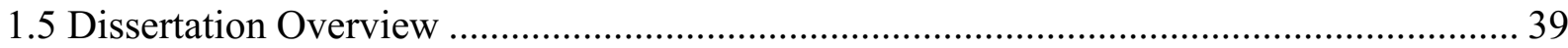

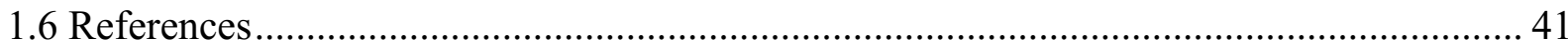

Chapter 2. Identification of MltG as a Potential Terminase for Peptidoglycan

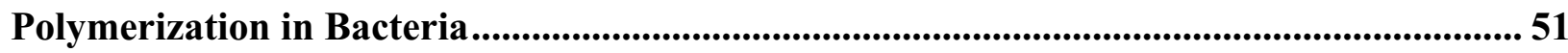

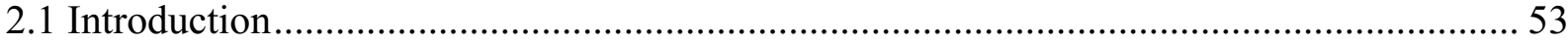

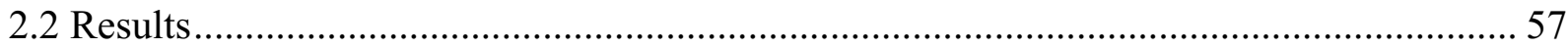

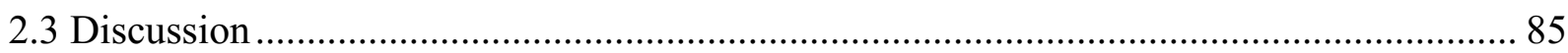

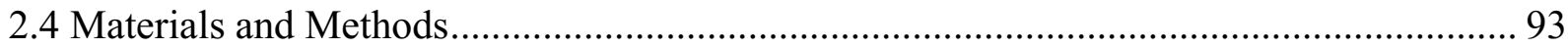

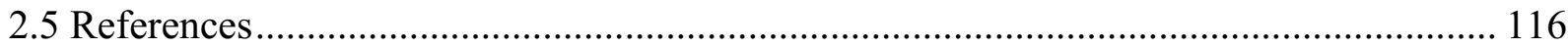

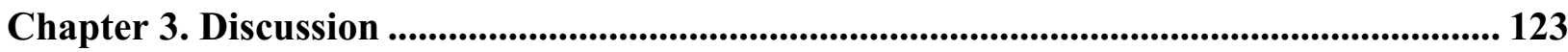

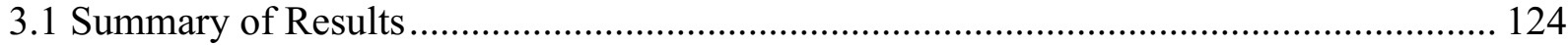

3.2 Role of MltG in Cell Wall Elongation......................................................................... 124

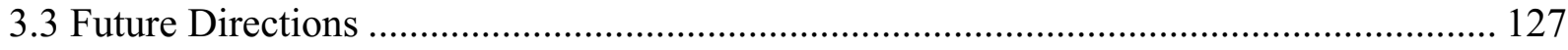

3.4 The Potential for LTs as Drug Targets ................................................................... 130

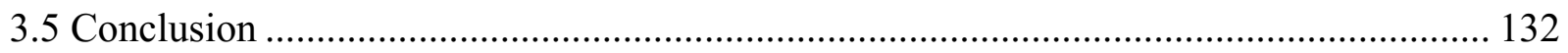

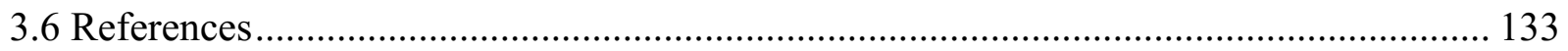




\section{Table of Figures}

\section{Chapter 1. Introduction to Lytic Transglycosylase in $E$. coli}

Figure 1.1. Schematic of the bacterial cell wall.............................................................. 5

Figure 1.2. The steps of PG biosynthesis....................................................................... 7

Figure 1.3. Structure of $E$. coli murein and sites of $\mathrm{PG}$ cleavage....................................... 10

Figure 1.4. Peptidoglycan glycosidases ......................................................................... 13

Figure 1.5 Reaction mechanism of Slt ....................................................................... 16

Figure 1.6. Schematic of PG synthesis and recycling pathways........................................ 22

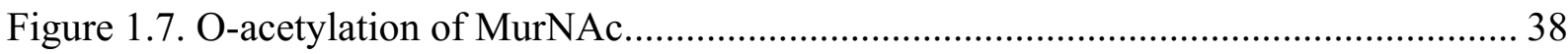

\section{Chapter 2 Identification of MItG as a Potential Terminase for Peptidoglycan Polymerization in Bacteria}

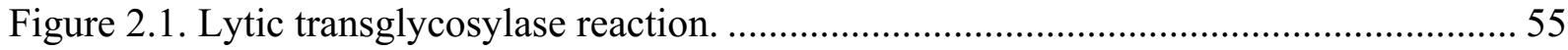

Figure 2.2. MltG overproduction is lethal in cells defective for PBP1b................................ 60

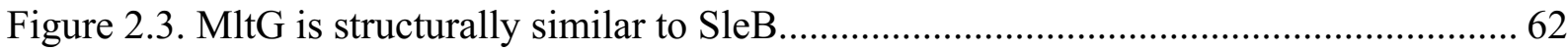

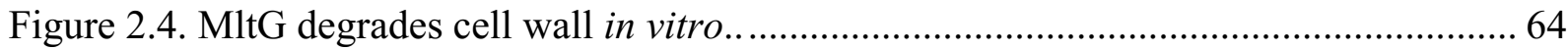

Figure 2.5. MltG is a lytic transglycosylase with endoglycosidase activity.........................66 66

Figure 2.6. MltG generates muropeptides with non-reducing termini.................................. 67

Figure 2.7. MltG is widely conserved in the bacterial domain......................................... 69

Figure 2.8. Conservation profiles of MltG and other LTs............................................. 70

Figure 2.9. MltG is an inner membrane protein............................................................ 72

Figure 2.10. Summary of muropeptide analysis of MltG defective cells. ............................. 75

Figure 2.11. Total ion chromatogram. Muropeptide Analysis WT (TB28) or $\Delta m l t G$ (RY36)..76

Figure 2.12. HPLC chromatogram. Glycan Strand Analysis WT (TB28) and $\Delta m l t G$ (RY36) 80

Figure 2.13. Bacterial two-hybrid interaction of MltG with PBP1b................................... 81

Figure 2.14. Nascent PG degradation by Slt and MltG following cefsulodin treatment......... 84

Figure 2.15. Model for MltG functioning as a PG terminase............................................. 87

Figure 2.16. Putative LysM domain in MltG..................................................................... 89 


\section{Dedication}

This dissertation is dedicated to

my mother

Louise Amy Yunck

Thank you for your endless love, support, guidance, and inspiration. 


\section{Acknowledgements}

I am deeply grateful to the many individuals who have supported me during my graduate work. First, I would like to thank my advisor Tom Bernhardt for his invaluable mentorship over the past several years. He is not only a brilliant bacterial geneticist but also a truly caring mentor and teacher. Through his guidance and support, he has helped me grow as a scientist and achieve more than I could have imagined.

I also had a wonderful dissertation advisory committee who provided valuable insight along the way: David Rudner, Simon Dove, and Suzanne Walker. Additionally, I would like to sincerely thank Simon Dove, Eric Rubin, Andrew Camilli, and Ann Hochschild for taking the time to join my defense committee. And many thanks to Chris Sham, Thao Truong, Jessica Bohrhunter, and Ghee Chuan for critical reading of my dissertation.

Former and current members of the Bernhardt lab have been a wonderful source of encouragement during my time in grad school. The lab has evolved and grown considerably over the years. During this time, I have had the pleasure of getting to know and learn from many wonderful people and brilliant scientists. Thank you to everyone in the Bernhardt lab. In particular, I would to thank my baymates, Ghee Chuan, Andrew, Ting, and our honorary baymate, Jessica, for their positive presence and stimulating conversations. I joined the lab at the same time as two other students, Mary Jane and Ghee Chuan, and I am grateful that we could go through this journey together. It wouldn't have been the same without you. To our younger graduate students, Thao, Patty, and Jessica - I am incredibly proud of you all and I wish you continued success.

I have also had the pleasure of working in a collaborating lab for part of my time in graduate school. For six months, I was a visiting student in the Pneumococcal Group led by 
Thierry Vernet at the Institute of Structural Biology in France. There, I had the honor of working with Cecile Morlot, who taught me an incredible amount in my short time there. I would like to express my sincere gratitude to Cecile and Thierry for welcoming me into their lab. My time there was truly transformative, scientifically and personally.

And lastly, I would like to send endless gratitude to my family and friends for their unwavering love, support, and encouragement. I owe everything I have accomplished to my mom who has instilled in me a love for learning and taught me the value of education. 
Chapter 1. Introduction to Lytic Transglycosylases in E. coli 


\subsection{CELL WALL BIOGENESIS}

Significance

The two decades between 1940 and 1960 have been described as the "golden age" of natural antibiotic discovery ${ }^{1}$. During this time, a core set of antibiotic scaffolds were established that have provided the foundation for most of our current antibiotic arsenal ${ }^{2}$. The global antibiotic market is still dominated by classes of antibiotics discovered in the mid-1900's ${ }^{1}$. With successive waves of resistant bacterial pathogens, the functional lifetime of well-worn chemical scaffolds has been repeatedly extended through synthetic chemical modifications ${ }^{1}$. For this reason, the time since 1960's has been considered the age of antibiotic tailoring and medicinal chemistry ${ }^{1}$. However, the utility of this tailoring strategy has had limited effectiveness, necessitating the discovery of new antibiotics or treatment strategies to fight the rising tide of drug-resistant infectious diseases ${ }^{1-4}$.

Over $70 \%$ of new antibacterials developed between 1980 and 2005 were built upon four chemical backbones: cephalosporins, penicillins, quinolones, and macrolides ${ }^{1}$. One of these core scaffolds, penicillin, represents the first naturally occurring antibiotic employed to treat bacterial infections ${ }^{5}$. Penicillin was first isolated by Alexander Fleming in 1928 after a fortuitous observation that contamination by Penicillium mold inhibited growth of bacterial strains ${ }^{5,6}$. However, within three years of the first clinical application of penicillin (1943), penicillinresistant strains began to emerge $\mathrm{e}^{2,5}$. Since that time, bacterial pathogens have continued to demonstrate their tremendous capacity to evade treatment efforts ${ }^{3,7}$.

Penicillin is one member of a broader class of antibiotics known as $\beta$-lactams that act by targeting the process of bacterial cell wall biogenesis, an essential, conserved, and unique pathway in bacteria ${ }^{8}$. The cell wall is one part of a multilayer, bacterial encasement collectively 
referred to as the cell envelope ${ }^{9}$. The cell envelope plays a crucial role in bacterial physiology as highlighted by the fact that approximately one-third of proteins in Escherichia coli are associated with the cell envelope ${ }^{10}$. However, approximately half of these proteins have unknown functions ${ }^{10}$. To sustain the ongoing search for for new targets and novel antibiotic compounds, significant research efforts have focused on broadening our understanding of cell wall biogenesis to uncover previously unidentified vulnerabilities in this essential structure.

\section{Overview of The Cell Wall}

The bacterial cell envelope is a complex structure made up of multiple layers ${ }^{9}$. The key structural and stress-bearing component of the bacterial cell envelope is the cell wall or sacculus $^{8}$. The cell wall provides the mechanical strength necessary to resist internal osmotic pressure amounting to several atmospheres, roughly equivalent to the pressure inside a car tire ${ }^{11}$. However, also similar to a tire, the cell can burst if the integrity of the cell wall is disrupted. This vulnerability is exploited by penicillin and other drugs that disrupt cell wall biogenesis. The cell wall is also essential for establishing bacterial cell shape. The incredible diversity of shapes and sizes observed across bacteria can be attributed to the varying approaches bacteria have adopted to build the cell wall ${ }^{12}$. The importance of cell wall architecture for bacterial morphology is highlighted by the fact that sacculi isolated from SDS-treated bacterial cells and visualized with electron microscopy largely retain the same dimensions and shape as those of the original cell ${ }^{8}$.

The specific components and characteristics of the layers that make up the cell envelope vary across bacterial species ${ }^{9}$. E. coli and other gram-negative bacteria have an envelope made up of an inner (IM) and outer plasma membrane (OM) with a thin cell wall sandwiched between them (Figure 1.1A). The region between the IM and OM is known as the periplasmic space. In 
contrast, gram-positive bacteria possess a thick cell wall surrounding the inner membrane and extending into the extracellular space (Figure 1.1A).

The bacterial cell wall is made up of peptidoglycan (PG), also referred to as murein, a heteropolymer made up of long glycan chains interconnected by short stem peptides (Figure 1.1B). Specifically, PG contains linear glycan strands with alternating units of $N$-acetylglucosamine (GlcNAc) and $N$-acetylmuramic acid (MurNAc) covalently linked by $\beta-1,4-$ glycosidic bonds ${ }^{8}$. These glycan strands are cross-linked to neighboring strands by short peptide chains that extend from the MurNAc sugars (Figure 1.1B). Together, this meshwork forms a continuous net-like macromolecule that encases the bacterial cell. Since PG surrounds bacteria in one continuous net, bacteria must dynamically expand and remodel the cell wall during growth and division ${ }^{8}$. Disruptions to the process of cell wall remodeling, such as those introduced by $\beta$ lactam antibiotics, can lead to a catastrophic breach in the cell wall and ultimately cause cell lysis. 


\section{A}

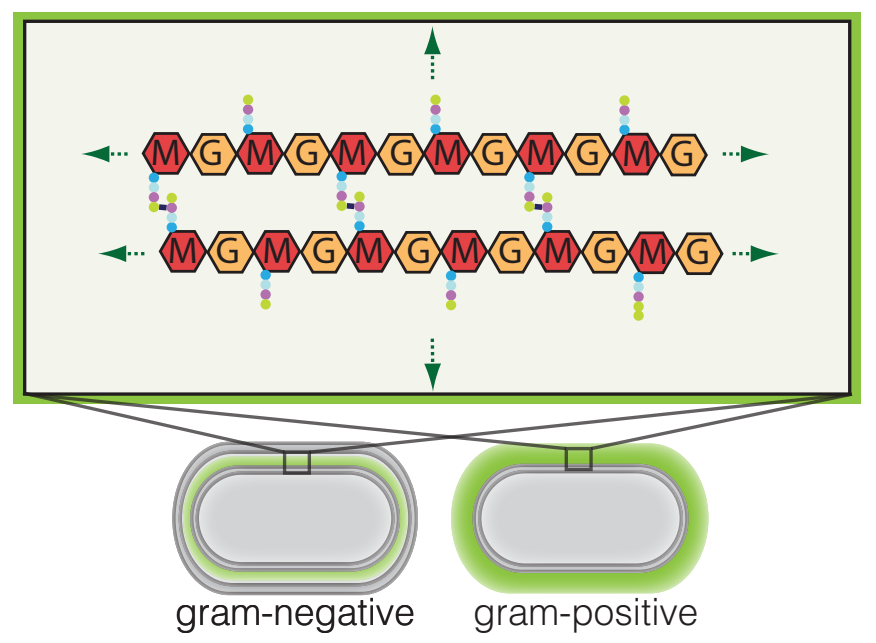

B

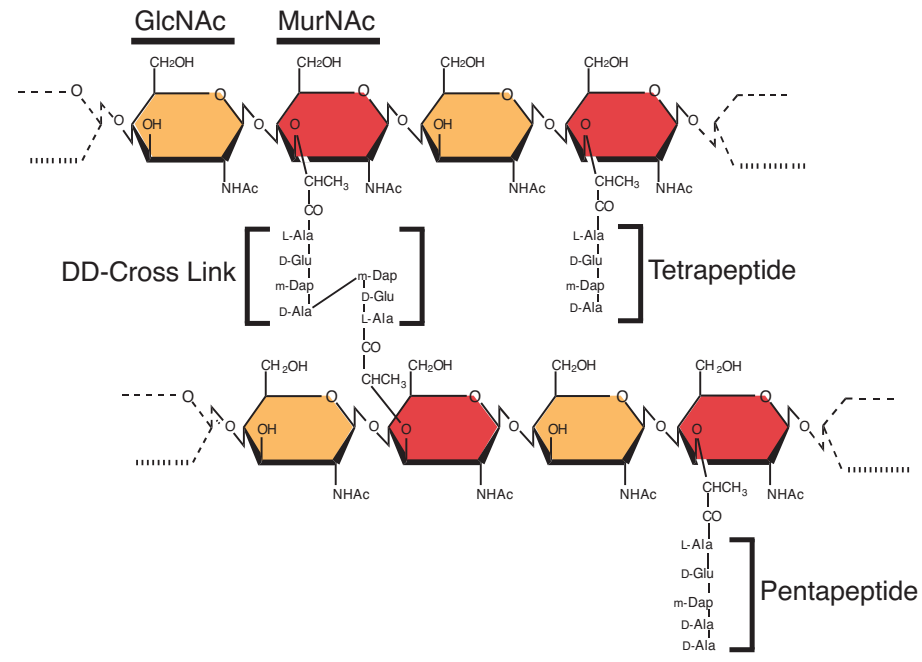

Figure 1.1. Schematic of the bacterial cell wall.

(A) Schematic of bacterial cells with the cell wall (PG layer) in green. Gram-negative cells have a relatively thin PG layer surrounded by an additional (outer) membrane. Above the cells is a schematic detailing the structure of PG, which continues in all directions to envelop the cell (green arrows). M ( $N$-acetyl-muramic acid) and $\mathrm{G}$ ( $N$-acetylglucosamine) make up the glycan strands, and the dots represent the attached peptides. 
Figure 1.1 (Continued). (B) Chemical structure of peptidoglycan with examples of common stem peptides and a DD-cross link between D-Ala and mDap (Adapted from Dijkstra (1997) ${ }^{13}$. The majority of cross-links in E. coli $(\sim 93 \%)$ are formed by D,D-cross links, with a small number of L,D-cross links between two mDap residues ${ }^{8}$.

\section{PG Biogenesis}

To expand the cell wall during bacterial growth and division, bacteria must synthesize new PG material and incorporate it into the pre-existing matrix ${ }^{14,15}$. The basic building blocks that make up peptidoglycan are initially synthesized in the cytoplasm (Figure 1.2A). First, nucleotide activated N-acetylmuramic acid (UDP-MurNAc) is generated from UDP N-acetylglucosamine (UDP-GlcNAc). MurNAc differs from GlcNAc by the presence of a lactyl group, which serves as the point of attachment for the stepwise addition of five amino acids: L-Ala, $\gamma$-D-Glu, (L)meso-diaminopimelic acid (DAP), D-Ala, and D-Ala. The resulting UDP-MurNAc-pentapeptide product is linked to a lipid carrier, undecaprenyl phosphate, yielding the membrane anchored precursor lipid I. Next, addition of GlcNAc to lipid I generates lipid II, which is subsequently flipped across the inner membrane into the periplasm by the flippase MurJ for the next step of PG biogenesis ${ }^{16}$. This lipid II disaccharide-pentapeptide monomer is the basic building block of PG.

The second stage of PG biosynthesis involves polymerization of the disaccharides of lipid II into long glycan strands which are then cross-linked into the pre-existing matrix via their stem peptides. Given that PG is made up of glycans and peptides, construction of the cell wall matrix depends on the formation of two types of covalent bonds: glycosidic and peptide/amide bonds ${ }^{8}$. Enzymes with transpeptidase and glycosyltransferase activity are thus necessary for weaving new PG monomers into the pre-existing meshwork. The primary PG synthases that carry out one or both of these activities are known as penicillin binding proteins (PBPs) ${ }^{8,14}$ (Figure 1.2B). 

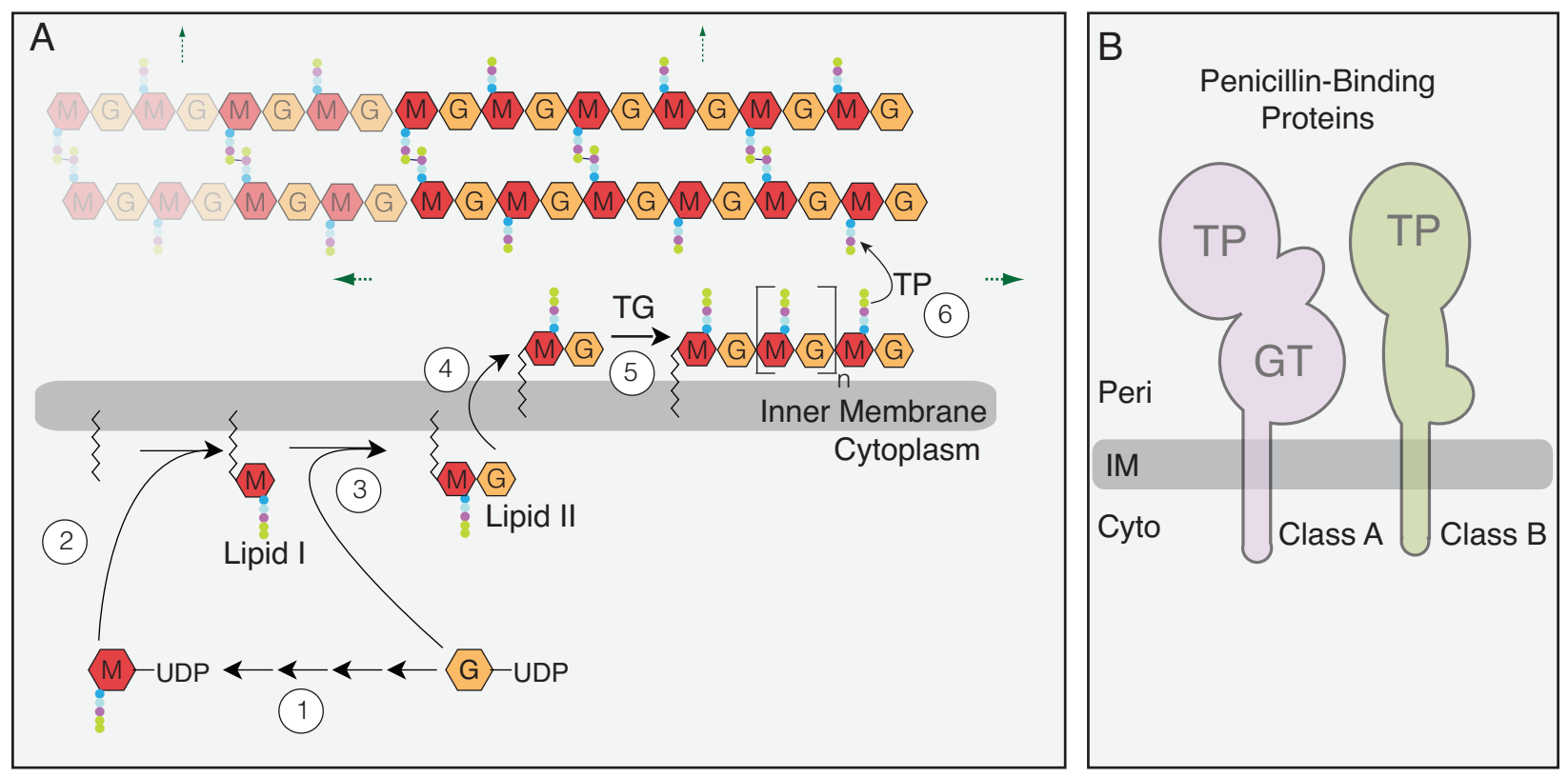

Figure 1.2. The steps of PG biosynthesis.

(A) The steps of PG biosynthesis. (1)UDP-GlcNAc is converted to UDP-MurNAc by several reaction steps represented here by multiple black arrows. (2)UDP-MurNAc attached to a pentapeptide side chain is then anchored in the inner membrane, generating lipid I.

(3) Attachment of GlcNAc to lipid I results in the formation of lipid II which can then be (4)flipped across the membrane and incorporated into the existing PG meshwork via (5)transglycosylation and (6)transpeptidation reactions. Adapted from Yang (2012) ${ }^{17}$.

(B) Domain structure of the PG synthases. Both classes of PBPs have a single transmembrane domain with a large catalytic domain in the periplasm. Adapted from Cho et al. (2014) ${ }^{18}$. Abbreviations: TG, transglycosylase; TP, transpeptidase. 
Penicillin binding proteins (PBPs) are named such for the ability of penicillin and other $\beta$-lactams to covalently bind and inhibit these enzymes ${ }^{19}$. PBPs can be clustered into either high molecular weight (HMW) or low molecular weight (LMW) families. HMW-PBPs include PBPs that are involved in PG synthesis, and can be further subdivided into the class A and class B PBPs (Figure 1.2B). Class A PBPs are bifunctional, possessing a glycosyltransferase domain to polymerize glycan strands and a transpeptidase domain to cross-link adjacent strands via their peptide chains. E. coli produces three class A PBPs: PBP1a, PBP1b, and PBP1c. PBP1a and PBP $1 \mathrm{~b}$ are believed to be the primary PG synthases in E. coli owing to their capacity to carry out the two reactions required for PG synthesis. Either PBP1a or PBP1b must be present in E. coli to survive, but cells are inviable if both are deleted simultaneously ${ }^{20-22}$. The only other known enzyme capable of polymerizing glycan strands in E. coli is a monofunctional glycosyltransferase, MtgA, believed to work with components of the cell division apparatus during septal PG synthesis ${ }^{23,24}$.

In contrast to their bifunctional counterparts, the class B PBPs, PBP2 and PBP3, are monofunctional transpeptidases (Figure 1.2B). Inactivation of PBP2 in E. coli produces spherical cells that eventually lyse, suggesting that PBP2 is specifically required for cell wall elongation ${ }^{25}$. In contrast, depletion of PBP3 causes cells to form long filaments due to an inability to generate division septa, implicating PBP3 in septal PG synthesis ${ }^{25}$. These findings suggest that the rod morphology of E. coli is governed by two independent modes of cell wall growth: 1) sidewall peptidoglycan biosynthesis during elongation, and 2) septal peptidoglycan biosynthesis during division. The balance between the activities of these two systems is essential for maintaining proper rod shape in E. coli $^{26,27}$. 


\section{PG Hydrolases}

To grow and divide, bacteria must carry out the delicate task of expanding, remodeling, and degrading PG, all while maintaining the integrity of this essential stress-bearing structure ${ }^{8}$. Modifications during cell wall remodeling are generated by a large repertoire of PG hydrolases that cleave bonds in the cell wall network. While the functions of the PG synthases are fairly well understood, much less is known about the roles of cell wall hydrolases in PG biogenesis and how these potentially lytic and dangerous enzymes are regulated ${ }^{28}$. Underlying their destructive potential, PG hydrolases are often referred to as autolysins due to their ability to cause cell lysis under certain conditions. Over 36 PG hydrolases have been identified in E. coli, each falling into one of 12 classes that recognize and cut a specific bond in $\mathrm{PG}^{28}$ (Figure 1.3 and Table 1.1).

The enzymes necessary to cleave PG act as either peptidases/amidases or glycosidases (Figure 1.3 and Table 1.1). PG peptidases exist in two forms. Carboxypeptidases remove the Cterminal amino acid of the peptide chain and endopeptidases hydrolyze peptide cross-links. Peptidases can further be designated as DD-, LD-, or DL-peptidases based on the stereochemistry of the bonds they cleave. For example, DD-endopeptidases of E. coli (PBP4, PBP7, and MepA) cleave the D-Ala $\rightarrow(\mathrm{D})$-mDap cross-links originally formed in the transpeptidation reaction carried out by HMW-PBPs. Finally, the $N$-acetylmuramyl-L-alanine amidases, commonly referred to as amidases, cleave stem peptides from glycan strands. E. coli has five known amidases, AmiA, AmiB, AmiC, AmiD, and AmpD. 


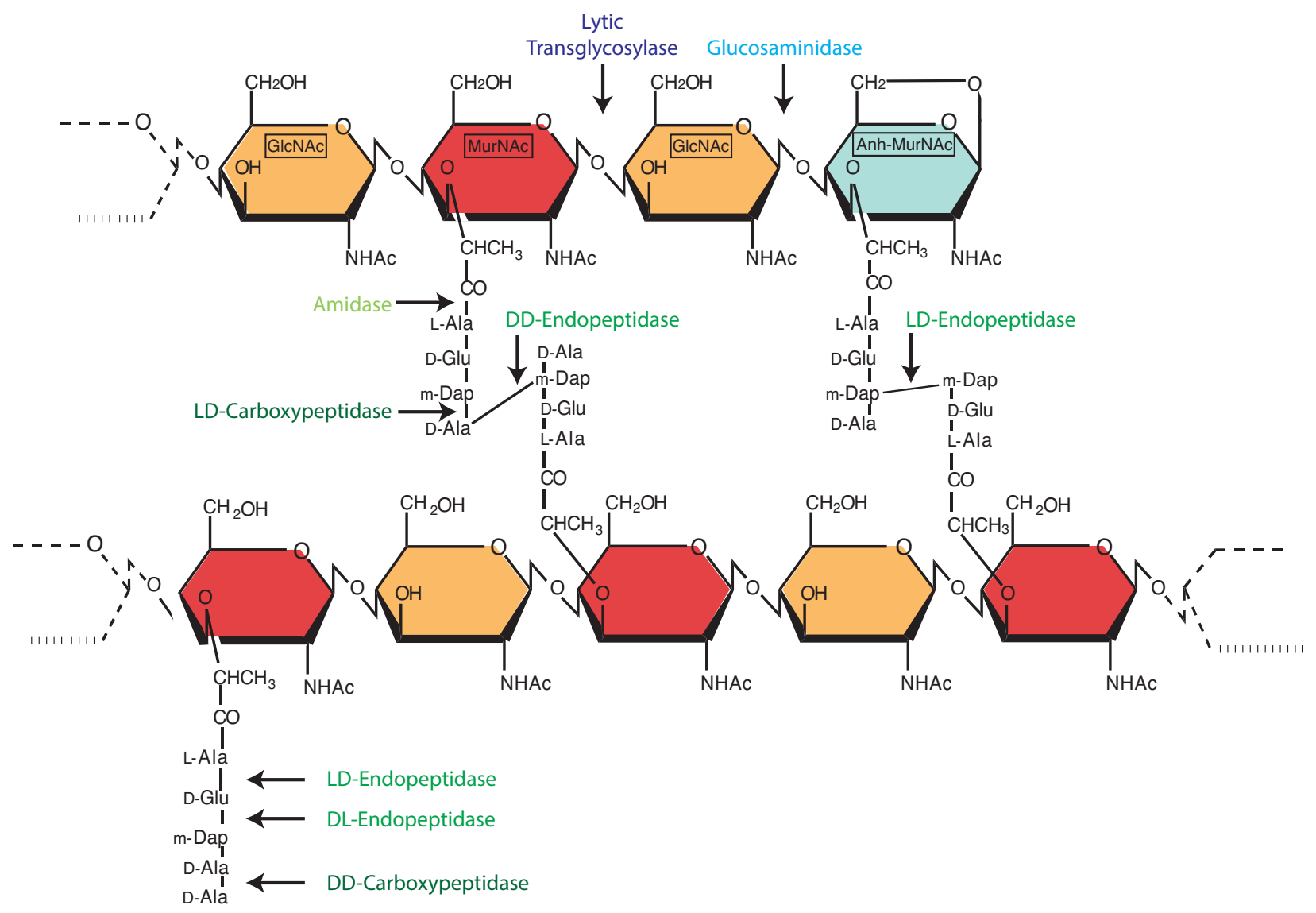

Figure 1.3. Structure of $E$. coli murein and sites of PG cleavage.

Enzyme classes are color-coded (Green, Peptidases and Blue, Glycosidases). Note: DLcarboxypeptidase is not shown.. Abbreviations: Anh-MurNAc, 1,6-anhydro- $N$-acetylmuramic acid. 
Table 1.1. Peptidoglycan hydrolases in E. coli. ${ }^{\text {a }}$

\begin{tabular}{|c|c|c|c|}
\hline Enzyme & Gene & Localization & Substrate(s) \\
\hline \multicolumn{4}{|c|}{ ß-N-Actylglucosaminidase } \\
\hline NagZ & nagZ & $\mathrm{C}$ & $\mathrm{SC}+\mathrm{PG}$ \\
\hline FlgJ & flgJ & $\mathrm{P}$ & PG \\
\hline \multicolumn{4}{|c|}{ Lytic Transglycosylases } \\
\hline Slt & slt & $\mathrm{P}$ & PG \\
\hline MltA & $m l t A$ & $\mathrm{OM}$ & $\mathrm{SC}+\mathrm{PG}$ \\
\hline MltB & $m l t B$ & $\mathrm{OM}$ & $\mathrm{SC}+\mathrm{PG}$ \\
\hline MltC & $m l t C$ & $\mathrm{OM}$ & PG \\
\hline MltD & $m l t D$ & $\mathrm{OM}$ & PG \\
\hline MltE & mltE & $\mathrm{OM}$ & $\mathrm{SC}+\mathrm{PG}$ \\
\hline MltF & $y f h D$ & $\mathrm{OM}$ & PG \\
\hline MltG & $y c e G$ & $\mathrm{IM}$ & PG \\
\hline \multicolumn{4}{|c|}{ DD-Carboxypeptidases } \\
\hline PB4b & $y f e W$ & IM & $\mathrm{SC}$ \\
\hline PBP5 & $\operatorname{dac} A$ & IM & $\mathrm{SC}+\mathrm{PG}$ \\
\hline PBP6 & $\operatorname{dac} C$ & IM & $\mathrm{SC}$ \\
\hline PBP6b & $d a c D$ & IM & PG \\
\hline \multicolumn{4}{|c|}{ DD-Endopeptidases } \\
\hline PBP7 & $p h b G$ & $\mathrm{P}$ & PG \\
\hline MepA & mер $A$ & $\mathrm{P}$ & $\mathrm{SC}+\mathrm{PG}$ \\
\hline MepS & $m e p S / s p r$ & $\mathrm{OM}$ & $\mathrm{SC}+\mathrm{PG}$ \\
\hline МерМ & mepM/yebA & IM & $\mathrm{SC}+\mathrm{PG}$ \\
\hline $\mathrm{MepH}$ & mepH/ydhO & $\mathrm{P}$ & $\mathrm{SC}+\mathrm{PG}$ \\
\hline \multicolumn{4}{|c|}{ DD-Peptidases } \\
\hline PBP4 & $d a c B$ & $\mathrm{P}$ & $\mathrm{SC}+\mathrm{PG}$ \\
\hline $\mathrm{AmpH}$ & $a m p H$ & IM & $\mathrm{SC}+\mathrm{PG}$ \\
\hline \multicolumn{4}{|c|}{ LD-Carboxypeptidases } \\
\hline LdcA & $\operatorname{ldc} A$ & $\mathrm{C}$ & $\mathrm{SC}$ \\
\hline \multicolumn{4}{|c|}{ LD-Transpeptidases } \\
\hline ErfK & erfK & $\mathrm{P}$ & PG \\
\hline YbiS & $y b i S$ & $\mathrm{P}$ & PG \\
\hline YcbB & $y c b B$ & $\mathrm{P}$ & PG \\
\hline YcfS & $y c f S$ & $\mathrm{P}$ & PG \\
\hline YnhG & $y n h G$ & $\mathrm{P}$ & PG \\
\hline \multicolumn{4}{|c|}{ Endoamidase } \\
\hline $\mathrm{MpaA}$ & траA & $\mathrm{C}$ & $\mathrm{SC}$ \\
\hline \multicolumn{4}{|c|}{ MurNAc-L-Ala amidases } \\
\hline AmiA & amiA & $\mathrm{P}$ & PG \\
\hline AmiB & $a m i B$ & $\mathrm{P}$ & PG \\
\hline AmiC & amiC & $\mathrm{P}$ & PG \\
\hline AmiD & amiD & $\mathrm{OM}$ & $\mathrm{SC}+\mathrm{PG}$ \\
\hline AmpD & $a m p D$ & IM & $\mathrm{SC}$ \\
\hline
\end{tabular}


Table 1.1 (Continued). Peptidoglycan hydrolases in E. coli. ${ }^{\text {a }}$

\begin{tabular}{llll}
\hline Enzyme & Gene & Localization & Substrate(s) \\
\hline $\begin{array}{l}\text { L-Ala-D/L-Glu epimerase } \\
\text { YcjG }\end{array}$ & $y c j G$ & $\mathrm{C}$ & $\mathrm{SC}$ \\
$\begin{array}{l}\text { D-Ala-D-Ala dipeptidase } \\
\text { DdpX }\end{array}$ & $d d p X$ & $\mathrm{C}$ & $\mathrm{SC}$ \\
\hline
\end{tabular}

a DD-peptidases show both DD-carboxypeptidase and DD-endopeptidase activities. C, cytoplasm; CM, cytoplasmic membrane; P, periplasm; OM, outer membrane; SC, soluble component (precursor, muropeptide, peptide or glycan chain); PG, isolated PG. Table adapted from VanHeijenoort $(2011)^{28}$.

PG cleaving enzymes that break glycosidic bonds are broadly referred to as glycosidases. These can be further subdivided into $\beta$ - $N$-acetyl-glucosaminidases that cleave the GlcNAc$(1 \rightarrow 4)$-MurNAc linkage and $\beta-N$-acetylmuramidases that cleave the MurNAc- $(1 \rightarrow 4)$-GlcNAc bond (Figure 1.3 and Figure 1.4). E. coli has one known glucosaminidase, NagZ. NagZ is a cytoplasmic enzyme required for recycling PG that has been internalized following PG turnover, but it is not essential for viability. The second family of glycosidases includes PG muramidases, lysozyme and lytic transglycosylases (LTs) (Figure 1.4). Although they cleave the same linkage in PG, they have different cleavage mechanisms and consequently generate different end products. Lysozyme catalyzes a hydrolysis reaction resulting in a reducing MurNAc sugar, whereas LTs carry out an intramolecular transglycosylation reaction, forming a 1,6-anhydro ring on MurNAc ${ }^{29-31}$ (Figure 1.4). Lysozyme is produced by animals or plants as a bacteriolytic mechanism of defense or by bacteriophage during penetration or lysis of bacterial cells. In contrast, lytic transglycosylases are found in most bacteria and are involved in normal cell wall metabolism (See Sections 1.2-1.4). 

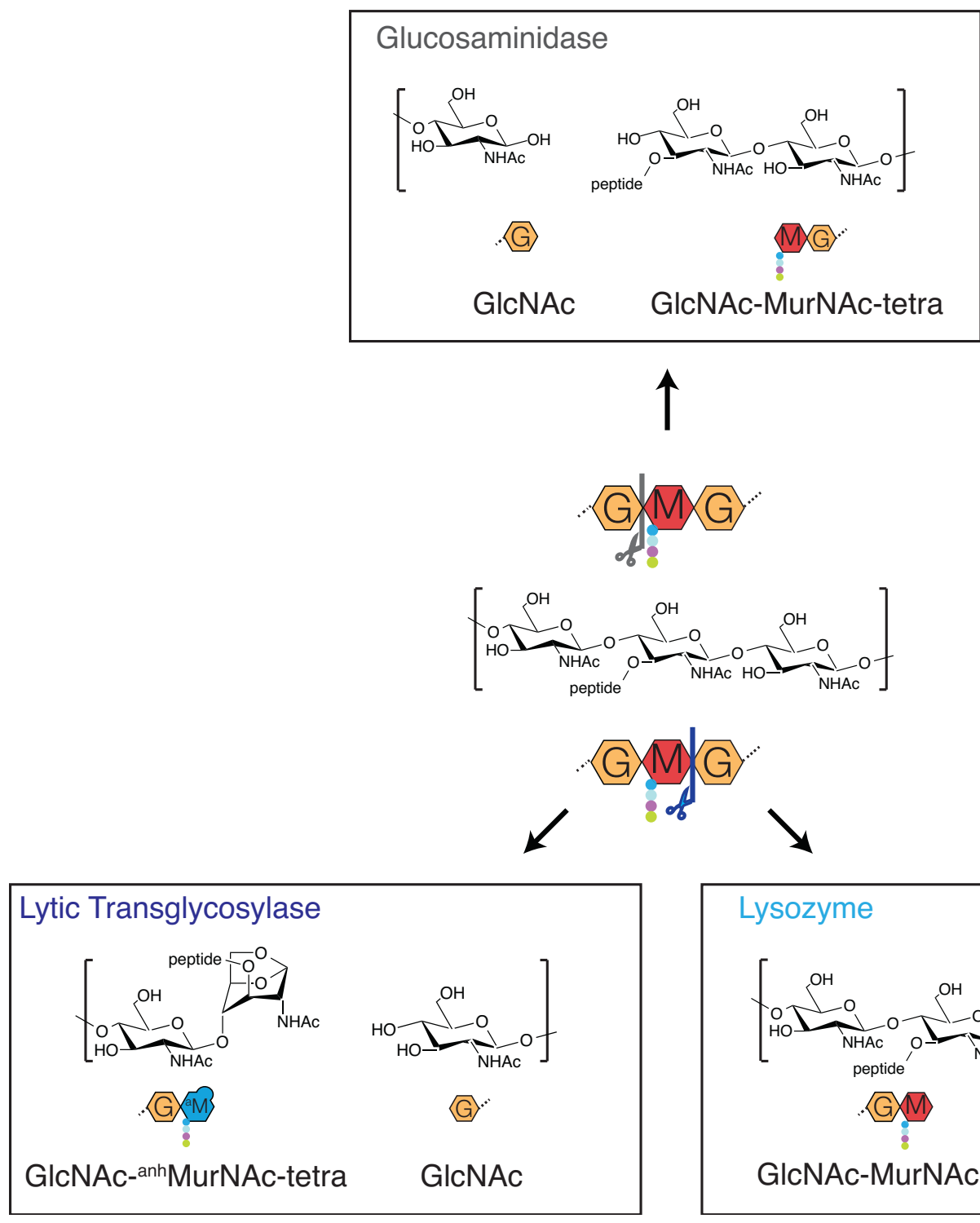

Lysozyme

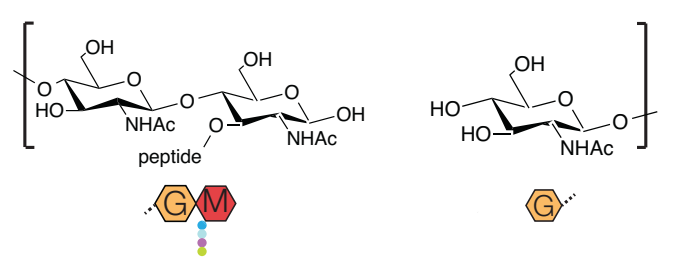

GlcNAc-MurNAc-tetra GlcNAc

\section{Figure 1.4. Peptidoglycan glycosidases}

$\beta$ - $N$-acetyl-glucosaminidases cleave the $\beta-1,4$ bond between GlcNAc and MurNAc linkage whereas $\beta-N$-acetylmuramidases (Lysozyme and Lytic Transglycosylase) cleave the $\beta-1,4$ bond between MurNAc and GlcNAc. Lysozyme catalyzes cleavage via a hydrolytic mechanism while lytic transglycosylases catalyze intramolecular glycosylation. 
PG hydrolases are believed to participate in a variety of essential processes, including cell wall expansion, division, and the turnover and recycling of mature $\mathrm{PG}^{28}$. However, it has been difficult to assign specific functions to PG hydrolases, especially in E. coli, largely due to the large number of functionally redundant members within each class of cell wall cleaving enzymes ${ }^{28}$. The systematic inactivation of multiple PG hydrolases is typically necessary to uncover phenotypes and assess protein function in vivo. Identification of sets of redundant enzymes within a given class is thus a crucial step in assaying function.

By identifying clusters of redundant genes, families of cell wall peptidases and amidases in $E$. coli have been demonstrated to be essential for cell wall expansion and division. One clearly defined function for PG hydrolases is the splitting of cell wall material shared between daughter cells during division ${ }^{28,32}$. The LytC-type amidases, AmiA, AmiB, and AmiC, are critical for septal PG splitting in E. coli ${ }^{32-34}$. A triple deletion mutant lacking AmiA, B, and C is unable to complete cell division, forming long cell chains connected by unsplit septal $\mathrm{PG}^{32}$. PG peptidases are also required to create space for the insertion of new PG material during cell elongation. Three endopeptidases in E. coli, MepS (Spr), MepH (YdhO), and MepM (YebA), implicated in the space making process are collectively essential for viability ${ }^{35,36}$. When all three are inactivated, cells are unable to cleave pre-existing cross-links for the incorporation of nascent PG and fail to expand their cell wall ${ }^{35,36}$. As demonstrated by these few examples, despite their lytic potential, PG hydrolases play a crucial role in the normal physiology of the bacterial cell. 


\subsection{OVERVIEW OF LYTIC TRANSGLYCOSYLASES}

Lytic transglycosylases (LTs) represent not only the predominant class of glycancleaving enzymes in E. coli, but also one of the most abundant classes of cell wall cleaving enzymes in E. coli (Table 1.1). Prior to work presented in Chapter 2, there were seven known LTs in E. coli. Six LTs are anchored to the outer membrane (MltA, MltB, MltC, MltD, MltE, MltF) and one localizes to the periplasm (Slt). Although LTs in E. coli have been wellcharacterized biochemically and structurally, little is known about their physiological roles. Individual LTs in E. coli are nonessential for viability under normal growth conditions ${ }^{37-44}$. Even a mutant lacking up to six LTs (Slt, MltA-E) is viable albeit with a minor chaining phenotype ${ }^{33,45}$. However, attempts to delete all seven have been unsuccessful, suggesting that lytic transglycosylase activity is essential for cell wall metabolism in E. coli ${ }^{44}$.

\section{Catalytic Mechanism}

During cleavage of glycosidic bonds, LTs carry out an intramolecular glycosylation reaction on the C-6 hydroxyl group of MurNAc leading to the formation of 1,6-anhydro$\operatorname{MurNAc}^{29}$ (Figure 1.5). This reaction is proposed to occur via a two-step substrate assisted catalytic mechanism ${ }^{46}$. First, a single catalytic residue, typically a glutamate, serves as a proton donor to the glycosidic oxygen between MurNAc and GlcNAc ${ }^{30,31}$. This leads to the formation of an oxocarbenium ion stabilized by the $N$-acetyl group of MurNAc, generating an oxazolinium intermediate $^{47}$. Second, the catalytic glutamate acts as a general base to abstract the proton from the C6-hydroxyl of MurNAc allowing an intramolecular nucleophilic attack on the $\mathrm{C} 1$ carbon to form a 1,6-anhydro ring ${ }^{30,31}$. 

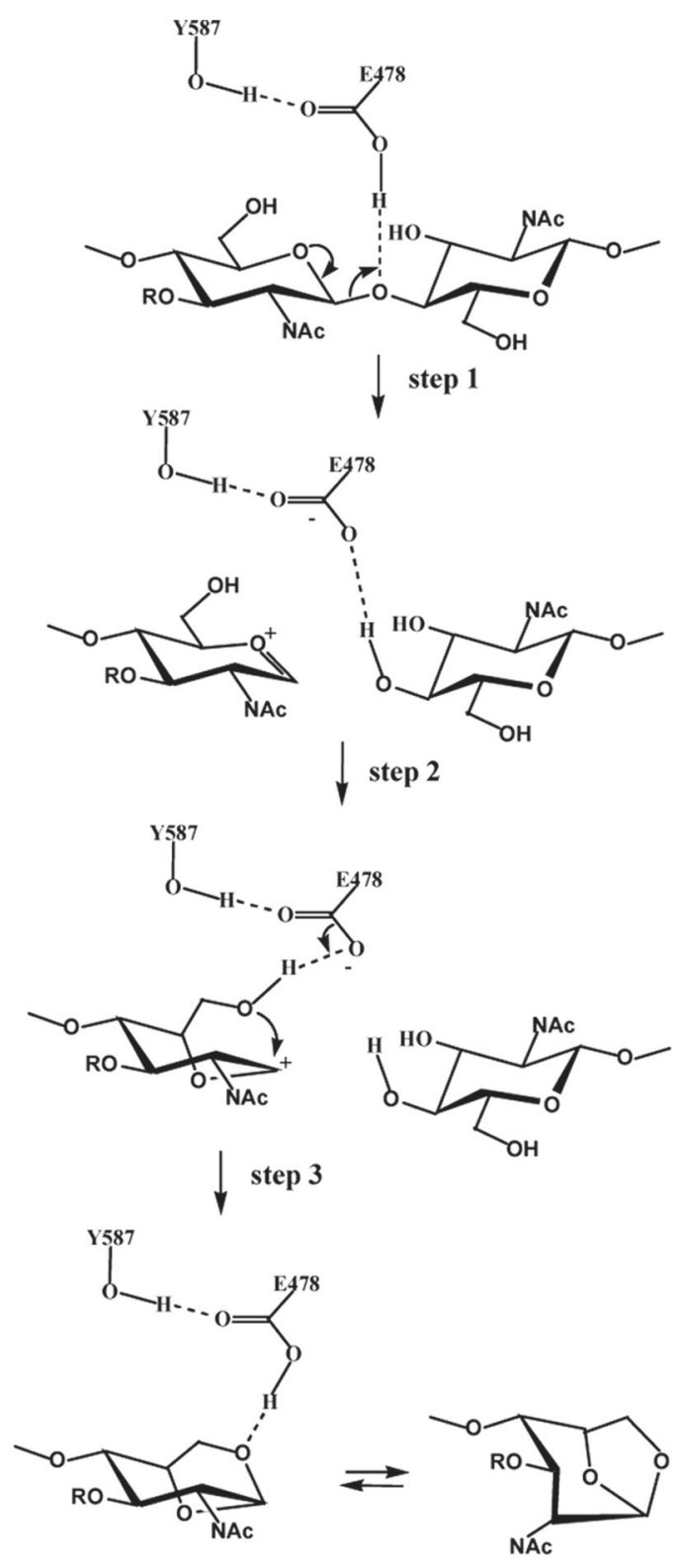

\section{Figure 1.5 Reaction mechanism of Slt}

LT reaction mechanism proposed by Thunnissen et al ${ }^{30}$. $\mathbf{R}$, stem peptide. Step 1 is the cleavage of the MurNAc- $(1 \rightarrow 4)$-GlcNAc bond with the formation of an oxocarbonium ion intermediate. Step 2 is an intramolecular nucleophilic attack on the $\mathrm{C}_{1}$ carbon of the oxocarbonium ion. Step 3 is the extraction of a proton by catalytic Glu478 and the formation of the 1,6-anhydroMurNAc- peptide. Figure reprinted from VanHeijenoort (2011) ${ }^{28}$ with permission of ASM. 
The active sites of Slt and lysozyme share a similar fold and also have a catalytic glutamate in common, reflecting their related roles as glycosidases ${ }^{30,31}$. However, there are also prominent differences between the two active sites that account for their disparate mechanisms of catalysis. In particular, lysozymes possess a conserved aspartate residue also required for catalysis. The aspartate is proposed to stabilize the oxocarbenium intermediate and increase its lifetime. This would allow the leaving group to diffuse away and a nucleophilic water molecule to enter the active site and complete hydrolysis, ultimately releasing MurNAc with a reducing end rather than an anhydro ring ${ }^{30,31}$.

\section{Substrate Specificities}

PG subunits containing an 1,6-anhydro-MurNAc are broadly referred to as anhydromuropeptides and can exist in two forms ${ }^{48}$. Some exist as free-floating species that are liberated from the PG matrix by lytic transglycosylases during cell wall turnover and recycling ${ }^{48}$. 1,6-anhydro-MurNAc residues are also directly incorporated into the cell wall at what would typically be the reducing (MurNAc) terminus of each glycan strand in E. coli ${ }^{29,49}$. These different anhydro populations reflect the varying substrate specificities and functions of LTs.

Anhydro-muropeptides are released from the matrix by the action of exo-lytic LTs that cleave anhydro-muropeptides, disaccharide by disaccharide, from the ends of glycan strands ${ }^{48}$. Most LTs in E. coli, namely Slt, MltA, MltB, MltC, MltD, and MltF are exo-acting enzymes (Table 1.2) ${ }^{31,48,50-52}$. Exo-enzymatic digestion is believed to occur in a processive manner, presumably with other regulatory mechanisms in place to prevent uncontrolled digestion of the cell wall ${ }^{53}$. The precise end of the strand from which they cleave has been in contention. In the case of Slt, biochemical evidence is consistent with an exo-enzyme that cleaves anhydro- 
disaccharides from the $\mathrm{N}$-acetyl-glucosamine terminus ${ }^{48,50}$, whereas structural evidence suggests cleavage starts at the non-reducing anhydro-MurNAc end of PG ${ }^{30,31,52}$.

Out of the initially identified lytic transglycosylases, MltE is the only endo-lytic LT, meaning that it preferentially cleaves at internal sites within glycan strands ${ }^{43,51,54}$. This is consistent with the series of muropeptide oligomers released by MltE following digestion of sacculi. Interestingly, a recent study showed that Slt, MltB, and MltD are not 100\% exo-lytic, and additionally release a small number of products arising from endo-lytic digestion, suggesting there is more flexibility in substrate utilization than originally thought ${ }^{51}$.

Exo-lytic vs endo-lytic activity as well as other substrate specificities are consistent with differences in the arrangements of the glycan binding pockets of each enzyme. Endo-lytic MltE and lysozyme, have open binding sites that can accommodate up to six disaccharides. Exo-LTs typically have bindings sites that can accommodate three to six disaccharides, with additional steric hindrances in place to restrict binding of longer strands. For example, Slt has a doughnutlike structure made up of a "superhelical" ring of alpha-helices and a separate lysozyme-like Cterminal domain ${ }^{31,52,55}$. The doughnut shape may impose substrate specificity by restricting accessibility to loose, uncrosslinked ends of glycan strands. Alternatively, the ring may be opened in certain circumstances to allow the doughnut to pass by peptide cross-links or to cleave internal glycan sites, although such a conformational change has yet to be directly observed. Such a change could be consistent with the small degree of exo-lytic activity that has also been observed with Slt ${ }^{51}$. Finally, LTs can also discriminate substrate based on the presence or absence of peptides or cross-links as summarized in Table 1.2. 
Table 1.2. Summary of substrate specificities of $E$. coli LTs

\begin{tabular}{|c|c|c|c|c|}
\hline & Exo vs Endo $^{51}$ & Cross-linked PG & $\begin{array}{l}\text { Glycans } \\
+ \text { Stem Peptides }\end{array}$ & $\begin{array}{l}\text { Glycans } \\
\text { - Stem Peptides }\end{array}$ \\
\hline$\overline{\text { Slt }}$ & $\begin{array}{l}\text { Exo } \\
(<\text { Endo })^{c}\end{array}$ & $+/-^{51}$ & $+31,50,51$ & -50 \\
\hline MItA & Exo & $+{ }^{51}$ & $+{ }^{51}$ & $+{ }^{50,56}$ \\
\hline MltB & $\begin{array}{l}\text { Exo } \\
(<<\text { Endo })\end{array}$ & $+{ }^{51}$ & $+{ }^{50,51}$ & -50 \\
\hline MltC & $\begin{array}{l}\text { Exo } \\
(<<<\text { Endo })\end{array}$ & $+{ }^{51}$ & $+51,53$ & -53 \\
\hline MltD & $\begin{array}{l}\text { Exo } \\
(<<\text { Endo })\end{array}$ & -51 & +51 & $?$ \\
\hline MtlE & Endo & $\begin{array}{l}+/-^{51} \\
\text { Pref for X-links? }\end{array}$ & $\begin{array}{l}\text { Inconsistent } \\
-{ }^{43} \text { and }+{ }^{51}\end{array}$ & $+{ }^{43}$ \\
\hline MltF & Exo & -51 & +51 & $?$ \\
\hline
\end{tabular}

$\begin{array}{|llccc|}\text { MltG }^{\mathbf{a}} & \text { Endo } & -{ }^{57} & { }^{57} & ? \\ \mathbf{R l p A}^{\mathbf{b}} & \text { Endo } & -{ }^{58} & -{ }^{58} & +{ }^{58}\end{array}$

${ }^{a}$ See Chapter 2.

${ }^{\mathrm{b}}$ LT activity of RlpA homolgue in E. coli not confirmed ${ }^{58}$.

${ }^{\mathrm{c}}$ Arrows (<, low activity; $<<<$, much lower endo activity relative to MltE); (+), Enzyme capable of cleaving substrate in header; (-), Enzyme incapable of cleaving substrate. (+/-), Enzyme has low activity with substrate. 


\section{Families}

LTs can be grouped into four families based on structure and conserved sequence motifs ${ }^{59}$. The first family is considered a large "superfamily" composed of five subfamilies, including Slt, MltC, MltD, MltE, MltF in E. coli. LTs in "Superfamily 1" are closely related to goose-type lysozyme, possessing similar active site topology and a conserved catalytic glutamate and serine 30,31,55,59. Members of Superfamily 1 are broadly conserved in gram-negative and gram-positive bacteria, as well as some bacteriophages ${ }^{59}$. MltA and MltB form the basis of their own families, Family 2 and Family 3 respectively, and are predominantly found in gram-negative bacteria. Meanwhile, Family 4 LTs are specifically found in bacteriophages. MltA “Family 2" LTs are distinct from other known LTs ${ }^{60}$. In contrast to the alpha-helical, lysozyme-like fold of Family 1, 3 and 4, MltA is a B-rich protein and resembles endoglucanase, an enzyme that cleaves the $\beta-(1,4)$-glycosidic bonds of cellulose ${ }^{60}$. Further, unlike other LTs, MltA activity relies on a catalytic aspartate (Asp308) rather than a glutamate residue ${ }^{60}$. Interestingly, MltA appears to be one of the few known LTs in E. coli, in addition to MltE, that can cleave both peptide-containing and denuded glycan strands ${ }^{43,56}$. The potential functional significance of this specificity is further addressed in Section 1.3. 


\subsection{PHYSIOLOGICAL FUNCTIONS OF LTS}

As with most classes of cell wall hydrolases, the functions of lytic transglycosylases have been difficult to unravel. Most appear to have overlapping functions in E. coli and further, individual LTs likely influence multiple cell wall processes. However, it can be inferred that LTs are required for particular cell wall processes based on the detection of anhydro-muropeptide byproducts. Although the following discussion of putative LT functions will focus primarily on E. coli, functional clues provided by homologous LTs in other organisms are also addressed. Additionally, a few instances are discussed where LTs have taken on specialized roles in specific bacterial species.

\section{PG Turnover and Recycling}

During bacterial growth, addition of new PG to the cell wall matrix is accompanied by the extensive release of pre-existing cell wall fragments (i.e. muropeptides) through a process called peptidoglycan turnover. The products released during cell wall turnover consist of monomeric muropeptides terminating in 1,6-anhydro-MurNAc ${ }^{61}$, indicating that lytic transglycosylases and endopeptidases play an important role in the turnover process. This is further supported by the fact that a mutant E. coli strain lacking three exo-lytic LTs, Slt, MltA, and MltB displays a $70 \%$ reduction in turnover ${ }^{62}$. In E. coli, over $40-50 \%$ of its existing PG is turned over per generation ${ }^{63}$. In most gram-positive bacteria, liberated muropeptides are primarily lost to the extracellular milieu ${ }^{64}$. In contrast, in E. coli and most other gram-negative bacteria, turnover is coupled to PG recycling where liberated anhydro-muropeptides are returned to the cytoplasm and broken down for reincorporation into new cell wall precursors (Figure 1.6) ${ }^{65}$. 


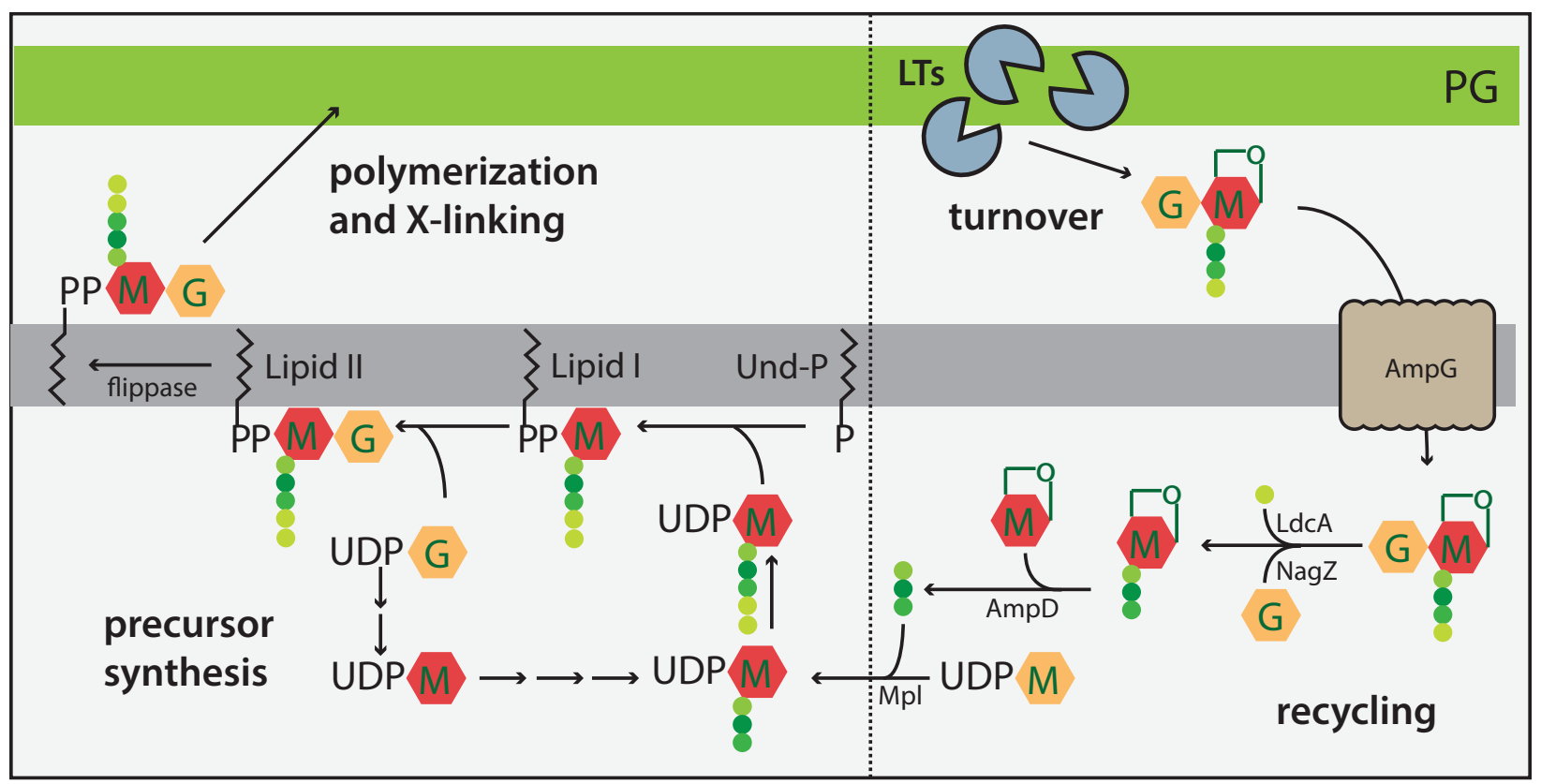

Figure 1.6. Schematic of PG synthesis and recycling pathways.

Anhydro-muropeptides arising from degradation by LTs are translocated into the cytoplasm by AmpG. Through a series of cytoplasmic reactions, anhydro-muropeptides are broken down into their component parts and reincorporated into PG precursors. Adapted from Cho et al (2014) ${ }^{18}$ 


\section{PG Recycling}

For cell wall recycling to occur, anhydro-muropeptides released by LTs must be translocated from the periplasm into the cytoplasm through the inner membrane permease, AmpG (Figure 1.6) ${ }^{66}$. Once in the cytoplasm, these fragments are broken down into their component parts through a series of reactions ${ }^{65}$. The cytoplasmic $N$-acetyl-glucosaminidase, NagZ, hydrolyzes imported disaccharides yielding GlcNAc and 1,6-anhydro-MurNAc ${ }^{67}$, ${ }^{68}$, while peptides are liberated from 1,6-anhydro-MurNAc by the cytoplasmic amidase, AmpD ${ }^{69}$. Additional enzymes downstream in the pathway convert 1,6-anhydro-MurNAc to GlcNAc, further break down the peptide chain, and re-channel these components to other enzymes for reuse ${ }^{65,70}$. Notably, multiple enzymes is this pathway (e.g., AmpG, AmpD) are specific for anhydro-muropeptides, ensuring that they can distinguish incoming cell wall material designated for recycling from the outgoing PG precursors dedicated to cell wall synthesis ${ }^{66,69}$.

\section{Induction of $\beta$-lactamase}

In some bacteria, bacterial cell wall recycling is intimately tied to the induction of antibiotic resistance mechanisms during $\beta$-lactam treatment ${ }^{71}$. One way bacteria neutralize the threat posed by $\beta$-lactams is through the production of $\beta$-lactamases that catalyze hydrolytic cleavage of the $\beta$-lactam scaffold, preventing it from engaging with target $\mathrm{PBPs}^{71}$. Many gramnegative bacteria produce $\beta$-lactamase $\mathrm{AmpC}$ in response to $\beta$-lactam treatment ${ }^{71}$. In certain bacteria (with the notable exceptions of $E$. coli and Shigella species), ampC is accompanied by the closely linked $a m p R$ locus encoding a transcriptional regulator (AmpR) that controls expression of $\mathrm{AmpC}^{71}$. 
Anhydro-muropeptides generated by LTs during turnover play a critical role in modulating the ampR-ampC system during ß-lactam treatment ${ }^{71}$. Mutants lacking LTs are not only defective in PG turnover, but also show reduced $a m p C$ induction ${ }^{62}$ and heightened sensitivity to $ß$-lactams ${ }^{72}$. In contrast, mutants lacking the AmpD amidase required for PG recycling accumulate anhydro-muropeptides in the cytoplasm, coinciding with the constitutive expression of $\operatorname{amp} C^{61,73}$. This has led to the model that elevated levels of anhydro turnover products in the cytoplasm cause $a m p C$ induction ${ }^{71,74}$. Consistent with this model, work in our lab has shown that $\beta$-lactam antibiotics trigger elevated levels of PG degradation by Slt, releasing large amounts of anhydro-muropeptides that are subsequently translocated into the cytoplasm ${ }^{18}$. In bacteria possessing the AmpR transcriptional regulator, cytoplasmic anhydro-muropeptides are believed to directly bind AmpR to modulate AmpC regulation ${ }^{61,74-76}$. LT turnover products can thus serve as messengers that relay the status of the cell wall to factors in the cytoplasm, activating protective measures to counteract the detrimental effects of $\beta$-lactams.

\section{Quality control}

The extensive cell wall turnover observed during ß-lactam treatment may ultimately be a byproduct of a peptidoglycan quality control function of LTs. ß-lactams covalently bind and inactivate the transpeptidase domain of PBPs, which effectively uncouples glycan strand polymerization from cross-linking reactions. This is proposed to cause the accumulation of uncrosslinked glycan strands that undergo rapid degradation by Slt, ultimately leading to a toxic and futile cycle of PG synthesis and degradation ${ }^{18}$. Slt-mediated degradation may be the cell's attempt at correcting faulty cell wall synthesis complexes where polymerization and crosslinking have become uncoupled. The rapid degradation of excess glycan material may prevent 
the formation of aberrant linkages in the matrix and may additionally release stalled PG synthesis complexes, facilitating the exchange of a damaged PBP for a functional one ${ }^{18}$. However, this quality control process becomes toxic when transpeptidation is inhibited by $ß$-lactams ${ }^{18}$.

\section{Host-Pathogen Interactions}

In addition to their role as intracellular messengers, PG turnover products can also act as extracellular messenger molecules, influencing host-pathogen interactions ${ }^{77,78}$. The tracheal cytotoxin (TCT) generated by Bordatella pertussis and Neisseria gonorrhoeae is the predominant monomeric product (GlcNAc- ${ }^{\text {anh }}$ MurNAc-Tetrapeptide) generated by most LTs. TCT can induce inflammation, damage ciliated epithelial cells, and stimulate the immune system by inducing interleukin expression ${ }^{77,79,80}$. Accordingly, animal and plant pathogens including Haemophilus influenza, Neisseria meningitidis, Shigella flexneri, and Pseudomonas syringae upregulate LT expression during host infection ${ }^{78}$. As further discussed in Section 1.3.III, LTs can also play an important part in virulence by aiding in the assembly of envelope-spanning secretion systems or acting as effector proteins translocated into host cells ${ }^{81,82}$.

\section{Cell Wall Expansion and Division}

The process of cell wall turnover and recycling is intimately linked to cell wall growth and division. In particular, turnover of peptidoglycan by lytic transglycosylases is believed to be tightly coordinated with insertion of new cell wall material ${ }^{83}$. LTs must additionally work together with other cell wall modifying enzymes, especially during the splitting of septal $\mathrm{PG}^{32,33}$. By coupling the activity of various cell wall modifying enzymes, bacteria can efficiently build up 
and break down their cell wall in a balanced manner while maintaining the integrity of the essential cell wall meshwork ${ }^{83}$.

\section{Septation}

During division in E. coli, PG synthesis is targeted to mid-cell to form a septum that upon splitting forms the poles of two new daughter cells. Although amidases have shown to be the key players in E. coli septal splitting ${ }^{32-34}$, LTs and endopeptidases also contribute to septal PG turnover $^{32,33}$. Deletion of at least three LTs in E. coli $(\Delta m l t C \Delta m l t D \Delta m l t E$ or $\Delta m l t A \Delta m l t B \Delta s l t)$ leads to a slight chaining phenotype that is further exacerbated upon deletion of additional LTs $(\Delta \text { slt } \Delta m l t A \Delta m l t B \Delta m l t C \Delta m l t E)^{33}$. However, even then the chaining phenotype of a $\Delta 5$-LT mutant (30-50\% of cells in chains of 3-8 cells long) is mild compared to that of a triple amidase deletion in E. coli ( $\triangle \mathrm{amiABC}, 90-100 \%$ cells forming chains of $6-25$ cells long) ${ }^{32}$. If amidase deletions are combined with deletions of LTs and endopeptidases ( $\Delta m e p A \Delta p b p 4 \Delta p b p 7)$, the division defect becomes very severe, with chain lengths of up to 100 cells $^{33}$. This is consistent with the idea that the LTs identified to date, along with endopeptidases, play a supportive role in division in E. coli.

In certain bacterial strains, lytic transglycosylases have been identified that are essential for septal PG cleavage. Pseudomonas aeruginosa encodes a recently confirmed LT, RlpA, required for cell division ${ }^{58}$. Consistent with a requirement for septation, deletion of $r l p A$ generates a chaining phenotype in $P$. aeruginosa ${ }^{58}$. RlpA is an outer membrane lipoprotein containing a C-terminal SPOR domain and an "RlpA-like" double psi beta barrel domain. SPOR domains are primarily found in division proteins and play a key role in recruiting division proteins to mid-cell ${ }^{84,85}$. During septal splitting, amidases at mid-cell strip stem peptides from 
glycan strands. Accordingly, in vitro assays have demonstrated that RlpA is only capable of cleaving glycan strands lacking stem peptides. Thus, upon recruitment to the division site, the active site of RlpA specifically recognizes and cuts "naked" glycan strands that have been preprocessed by septal amidases ${ }^{58}$. A homologue of RlpA with significant sequence similarity is also present in E. coli but enzymatic activity was not detected in vitro ${ }^{58}$.

Interestingly, structural modeling of RlpA shows that it has similarities to MltA of $E$. coli, including B-rich domains and a conserved catalytic aspartate ${ }^{58}$. As previously discussed, these features set MltA apart from other lysozyme-like LTs. Further, like RlpA, MltA is one of the few known LTs in E. coli capable of degrading denuded glycan strands. MltA homologues in other organisms have been linked to septation, including $\operatorname{LtgC}$ in $N$ gonorrhoeae ${ }^{86}$ and GNA33 in N. meningitidis ${ }^{87}$. Together, this suggests MltA-like "Family 2" LTs may have a conserved role in bacterial cell division.

\section{Cell Wall Expansion}

To expand the cell wall, bacteria must achieve a net increase in cell wall material each generation despite high PG turnover. The importance of turnover for cell wall expansion and how bacteria coordinate the two processes is unknown. One model by which bacteria could attain net cell wall expansion coupled with turnover is described in the "three-for-one model" $8,83,88,89$. In this model, expansion of the cell wall is achieved through a multi-enzyme complex containing endopeptidases, LTs, and PG synthases. This complex synthesizes and insert a triplet of new glycan chains in place of one pre-existing chain, called the docking strand. For this to occur, cross-linkages in the cell wall are cleaved by an endopeptidase, creating space for insertion of the incoming material. After new chains are cross-linked into place, the single, older 
strand is degraded by an exo-lytic LT. This mechanism could account for how bacteria couple cell wall expansion to turnover while maintaining cell wall integrity ${ }^{8,83}$. The growth strategy described in this model is considered a "make-before-break" strategy, where synthesis precedes bond hydrolysis ${ }^{8}$. It has alternatively been proposed that space-making hydrolases degrade preexisting material prior to the insertion of newly synthesized $\mathrm{PG}^{90,91}$. Further experimental work is required to evaluate these models of cell wall insertion.

Regardless of the mechanism, the process of glycan strand cleavage must be carefully coordinated with glycan strand polymerization to avoid detrimental lesions in the PG matrix. As proposed in the three-for one model, multi-enzyme complexes that possess both PG synthases and cell wall cleaving enzymes may help ensure that PG hydrolases only degrade cell wall where new PG is actively added. Consistent with this model, a number of protein-protein interactions have been detected between LTs and PBPs (Table 1.3) ${ }^{92-97}$.

Since most LT - PBP interactions proposed are based on affinity chromatography data, it is not clear to what extent these interactions are direct or bridged by other factors ${ }^{98}$. For example, MltA retains PBP1b on an affinity chromatography column, but surface plasmon resonance experiments show that they do not directly interact ${ }^{92}$. Instead an additional periplasmic factor, MipA, was discovered to mediate complex formation between MltA and PBP1b ${ }^{92}$. Further, these studies do not take into account the potential topological separation of LTs in the outer membrane from PG synthases in the inner membrane, which could restrict their potential for interaction in vivo, perhaps even as a means of regulation (See Section 1.4). Thus, further research is required to evaluate the physiological significance of the detected interactions between PBPs and LTs. 
Table 1.3. Interactions between LTs and PBPs in E. coli $^{\text {a }}$

\begin{tabular}{|c|c|c|c|c|c|}
\hline Enzymes & PBP1a & PBP1b & PBP1c & PBP2 & PBP3 \\
\hline SIt & & $\mathrm{AC}^{93,99}$ & $\mathrm{AC}^{93,99}$ & $\mathrm{AC}^{93,99}$ & $\mathrm{AC}^{93,99}$ \\
\hline $\begin{array}{l}\text { MltA } \\
\text { MipA }\end{array}$ & & $\begin{array}{l}\mathrm{AC}^{92,100,101} \\
\mathbf{A C}^{92} \\
\text { SPR }^{92,102}\end{array}$ & $\mathrm{AC}^{92}$ & $\mathrm{AC}^{92}$ & $\mathrm{AC}^{92}$ \\
\hline MltB & & $\mathrm{AC}^{93}$ & $\mathrm{AC}^{93}$ & & $\mathrm{AC}^{93}$ \\
\hline
\end{tabular}

abbreviations: AC, affinity chromatography; SPR, surface plasmon resonance Adapted from Zapun et al (2011) ${ }^{98}$ 


\section{Glycan strand termination}

Beyond functioning as space-makers, lytic transglycosylases are also believed to work closely with PBPs to terminate glycan chain polymerization during PG synthesis (See Chapter 2). Although most anhydro-muropeptides are liberated by exo-lytic LTs during PG turnover, 1,6anhydro-MurNAc residues are also incorporated into the PG matrix at what would typically be the reducing (MurNAc) terminus of each glycan strand (Figure 1.2). These anhydro-caps make up 3 to $4 \%$ of the total muropeptide composition of E. coli ${ }^{103,104}$. Pulse-labelling experiments have shown that this anhydro cap is introduced shortly after or concomitantly with termination of polymerization ${ }^{105,106}$, suggesting that a LT acts early in cell wall synthesis and processes nascent glycan material. However, an E. coli strain lacking up to six LTs ( $\Delta$ slt $\Delta m l t A-E)$ shows little reduction in the anhydro content of the PG matrix ${ }^{33}$, indicating that another LT is responsible for cleaving newly synthesized PG. LT cleavage of nascent glycan chains may help terminate PG polymerization and permit release of new PG strands from the PG synthesis complex. Additionally, this termination activity may be important for determining the distribution of glycan chain lengths in the cell. How bacteria determine glycan chain length and the importance of doing so is unknown ${ }^{28}$ and has been a major focus of my thesis work, as described in Ch .2.

\section{Specialized LTS}

In addition to contributing to the core processes of PG metabolism, LTs are also dedicated to specialized functions in bacteria ${ }^{107}$. In sporulating bacterial species (Bacillus and Clostridium), for example, lytic transglycosylases play a key role in metabolizing PG during endospore formation and germination. Additionally, in many bacteria, specialized LTs are involved in the insertion of trans-envelope structures ${ }^{82,107,108}$. Natural pores $(\sim 2 \mathrm{~nm})$ within the 
cell wall meshwork can accommodate the passage of globular proteins of up to 50 to $100 \mathrm{kDa}$, but these spaces are not large enough to allow the assembly of large complexes ${ }^{109,110}$. Due to this size limitation, large trans-envelope complexes must find ways to circumvent the cell wall barrier. These include type II, III, and IV secretion systems as well as type IV pili and flagella $82,108,111$. To act as space-makers during assembly of trans-envelope complexes, cell wall cleaving enzymes, often lytic transglycosylases, are encoded in gene clusters associated with regulation and assembly of these structures ${ }^{108}$. These LTs act at the desired site of assembly to generate a localized opening within the cell wall ${ }^{108}$. Among LTs with specialized functions are several "Family 1 LTs" involved with conjugation and secretion systems (VirB1, Agrobacterium tumefaciens; IpgF, Shigella flexneri, AtlA in Neisseria gonorrhoeae) and "Family 3 LTs" involved in flagella and pili formation (PleA, Caulobacter crescentus, BfpH, E. coli EPEC) $46,108,112$. Deletions of certain LTs have accordingly been associated with defects in motility, biofilm formation, and virulence ${ }^{113}$. 


\subsection{REGULATION OF LTS}

As established in the previous section, lytic transglycosylases have a diverse range of essential roles in bacterial cells. To accomplish these important tasks, the activity of LTs must be under tight spatial and temporal control to ensure that they cleave at the right place and time. Mis-regulation of their activity could have disastrous effects for the cell, as observed with the morphological defects and cell lysis associated with elevated expression of $\mathrm{Slt}^{114}, \mathrm{MltA}^{39}$, $\mathrm{MltB}^{40}$, and $\mathrm{MltD}{ }^{42}$. Although much remains to be learned about how bacteria control lytic enzymes, several regulatory strategies to control LT activity have been described or predicted as summarized below.

\section{Transcriptional Control}

Specialized lytic transglycosylases associated with secretion systems tend to occur in coregulated gene clusters encoding other system components, ensuring that the LT is produced only when required ${ }^{82,108}$. One well characterized instance of this is the VirB1 LT encoded in an operon associated with the Type IV Secretion Systems (T4SS) of Agrobacterium tumefaciens and Brucella suis ${ }^{115},{ }^{82,116}$. In Caulobacter crescentus, expression of a LT-encoding gene, pleA, is required for assembly of the motility apparatus ${ }^{117}$. Expression of pleA occurs concurrently with the appearance of pili and flagella, indicating that this enzyme is temporally regulated with development ${ }^{117}$. In spore-forming bacteria, PG hydrolases critical for spore-formation and germination are controlled at the transcriptional level by sporulation-specific sigma factors that limit production of these enzymes to particular stages of the differentiation process ${ }^{111,118}$. Finally, LTs have also been shown to be upregulated during infection by certain bacterial pathogens, suggesting that some LTs are specifically important during pathogenesis ${ }^{77}$. 


\section{Post-translational Control}

Multi-enzyme complexes

Lytic transglycosylases likely work in conjunction with other enzymes, including PG synthases and hydrolases. Interactions of components in a multi-enzyme complex may help spatially control and temporally coordinate the activity of PG-cleaving enzymes ${ }^{82}$. Additionally, interactions may directly modulate LT activity, either to promote or suppress cell wall cleavage. For example, in Mycobacteria tuberculosis, resuscitation promoting factor B (RpfB) is a LT that interacts with endopeptidase, RipA, to hydrolyze $\mathrm{PG}^{97}$. They work in complex synergistically and promote enhanced hydrolysis during septation ${ }^{97}$. RipA additionally interacts with PBP1 during septum formation, which has been shown to suppress $\mathrm{PG}$ hydrolysis of the RipA/RpfB complex in vitro ${ }^{97,119}$. Such interactions would ensure that PG cleavage does not commence until septal formation is complete. Interestingly, a synergistic relationship between an E. coli DD-endopeptidase, PBP8, and Slt has also been observed in vitro ${ }^{93}$. Slt is also stimulated by heat-inactivated PBP8, suggesting this stimulation of LT activity occurs through direct proteinprotein interactions rather than PBP8 pre-processing of the PG substrate ${ }^{93}$. A similar synergistic effect has also been observed between SpoIID and and SpoIIP in B. subtilis, suggesting that such partnerships could be a conserved mechanism to promote the concerted action of two PG cleaving enzymes at the same place and time ${ }^{120}$.

As discussed previously, LTs in E. coli have been observed in complex with many PG synthases ${ }^{92-94}$. Associations between an LT and members of the PG synthesis complex have also been indirectly demonstrated in Caulobacter. In C. crescentus, MltA and its interacting protein MipA display a banded localization pattern similar to PBP2 ${ }^{121}$. The subcellular localization pattern of MltA, MipA, and PBP2 are dependent on MreC, an integral membrane protein 
essential for cell wall elongation in rod-shaped bacteria, including E. coli ${ }^{122,121}$. Consistent with affinity chromatography studies in $E$. coli ${ }^{92}$, Caulobacter MltA and MipA interact with each other and further interact with MreC in bacterial two hybrid studies ${ }^{121}$. Based on this data, it has been proposed that $\mathrm{MreC}$ is required for maintaining the spatial localization of $\mathrm{PG}$ biosynthetic complexes involving PG synthases and hydrolases ${ }^{121}$.

Although it has long been hypothesized that hydrolases and synthases are coordinated within multi-enzyme complexes, the functional significance of such complexes remain unclear. It was often predicted such a complex would be necessary to confine hydrolysis to sites of PG synthesis, but in the case of PBP1-RipA, in vitro activity of RipA is suppressed rather than promoted when it is in association with PBP1 ${ }^{97}$. Multi-enzyme cell wall complexes have yet to be reconstituted in vitro and investigations into the consequences of disrupting multi-enzyme complexes in vivo are required. Consequently, although the idea of multi-functional PG modifying complexes is appealing, much remains to be learned about the physiological role and components of these multi-enzyme complexes.

In the case of specialized LTs, in addition to transcriptional co-regulation, protein-protein associations with secretion system components are believed to restrict LT activity to desired assembly sites. Returning to the example of the LT VirB1 involved in type IV secretion, VirB1 interacts with the VirB4 ATPase, which is important for substrate translocation ${ }^{82,123}$. Additionally, some specialized LTs lack a discernible Sec secretion signal, suggesting that LTs may be secreted by their associated secretion systems ${ }^{82}$. For example, in the plant pathogen Pseudomonas syringae, the LTs HrpH and HopP1 are substrates for its type III secretion system and can be transported into hosts as effector proteins ${ }^{81}$. 


\section{Subcellular Localization}

Cell wall cleaving enzymes can be spatially controlled through recruitment to specific subcellular sites ${ }^{82}$. A key example in E. coli is the recruitment of the amidases and their corresponding regulators to mid-cell during cell division ${ }^{124,125,126}$. Specific localization of enzymes can be accomplished i) by the inclusion of accessory targeting domains within the protein, ii) recruitment through protein-protein interactions, and/or iii) recruitment via binding of specially-localized PG substrate. The localization patterns of the LTs in E. coli have yet to be studied. However, RlpA in P. aeruginosa is an example of a LT that specifically localizes to division septa ${ }^{58}$. The SPOR domain in RlpA recognizes and binds denuded glycan chains enriched at septa, helping target RlpA to its desired site of activity ${ }^{58,127}$. This specificity for uncrosslinked strands also demonstrates that pre-processing by other cell wall cleaving enzymes can modulate LT activity by restricting when or where a specific substrate is available. Precise targeting of LTs is also observed with motility and secretion systems ${ }^{82}$. In addition to being temporally regulated via gene expression, the lytic transglycosylase PleA is observed to colocalize with flagella and pili at the distal pole of swarmer cells ${ }^{117}$.

Another potential mode of spatial regulation can be achieved through the distribution of LTs across the various layers of the cell envelope. It was originally hypothesized that PG hydrolases were controlled by their topological distribution owing to the fact in E. coli, most LTs are anchored in the OM as lipoproteins, while the PG synthases are localized to the $\mathrm{IM}^{8,15}$. This orientation may promote synthesis and hydrolysis on opposing sides of the cell wall, such that nascent PG remains protected from degradation and only mature PG is broken down during turnover. Alternatively, outer membrane LTs could be drawn in closer proximity to the cell wall by association with PG synthesis complexes, consistent with protein interaction data ${ }^{15,39}$. This 
would promote PG turnover in regions where PG synthesis is active. However, as previously mentioned, further genetic and biochemical evidence will be required to assess this model. Additionally, this cannot account for regulation of periplasmic or the few inner membrane localized LTs in E. coli.

\section{Proteolytic Processing}

Some bacteria depend on proteolytic processing for activation of cell wall modifying enzymes ${ }^{111}$. For example, the amidase/glucosaminidase Atl in Staphylococcus aureus is synthesized and secreted as an inactive proenzyme and must be processed to generate a mature enzyme $^{111}$. In addition to modulation by protein-protein interactions, RipA in Mycobacteria requires proteolytic processing to become active, thus demonstrating multiple layers of regulation $^{119}$. Interestingly, the lipoprotein MltB in E. coli is also known to undergo proteolytic cleavage resulting in a soluble variant called Slt $35^{40}$. Is it not known if this proteolysis serves a physiological function in vivo.

\section{Inhibitory factors}

Some bacteria generate specific protein inhibitors that are proposed to suppress LT activity. One such protein, Ivy, is encoded by $y f k E$ in $E$. coli and was originally identified as an inhibitor of vertebrate lysozyme ${ }^{128}$. In addition to inhibiting lysozyme, Ivy and its paralogues in P. aeruginosa can strongly inhibit MltB ${ }^{129}$. Ivy homologues can only be found in Proteobacteria that do not use O-acetylation, a form of substrate modification, as a regulatory strategy (See 1.4.III) ${ }^{129}$. Given this, it has been suggested that one physiological function of Ivy is to control the activity of LTs in the periplasm of Gram-negative bacteria that lack O-acetylated PG ${ }^{129,130}$. 
Other distantly related homologues of Ivy include MliC (membrane bound lysozyme inhibitor) and PliG (periplasmic lysozyme inhibitor) in E. coli, both of which have been shown to inhibit lysozyme. Similar to Ivy, it is predicted that these lysozyme inhibitors may normally function in regulating LTs, but this remains to be evaluated ${ }^{130}$.

\section{Substrate Modification}

\section{O-acetylation}

In addition to transcriptional or post-translational regulatory strategies, some bacteria also chemically modify their peptidoglycan to prevent degradation by cell wall cleaving enzymes ${ }^{130}$. During maturation of the cell wall, a range of Gram-positive and Gram-negative species chemically modify polymerized peptidoglycan through O-acetylation of the C-6 hydroxyl group of $\operatorname{MurNAc}^{131}$ (Figure 1.7). Since LTs require a free C6-OH group to catalyze the formation of a 1,6-anhydro ring, they are unable to cleave O-acetylated MurNAc. By controlling the degree of O-acetylation within the matrix, bacteria can control endogenous lytic transglycosylases and also provide a layer of protection against exogenous lysozymes ${ }^{130}$. Beyond O-acetylation, glycan modifications that can regulate LT activity also include $\delta$-lactam formation in Gram-positive spore cortex $\mathrm{PG}^{14}$. It is not clear how the introduction of these various modifications may be regulated. 


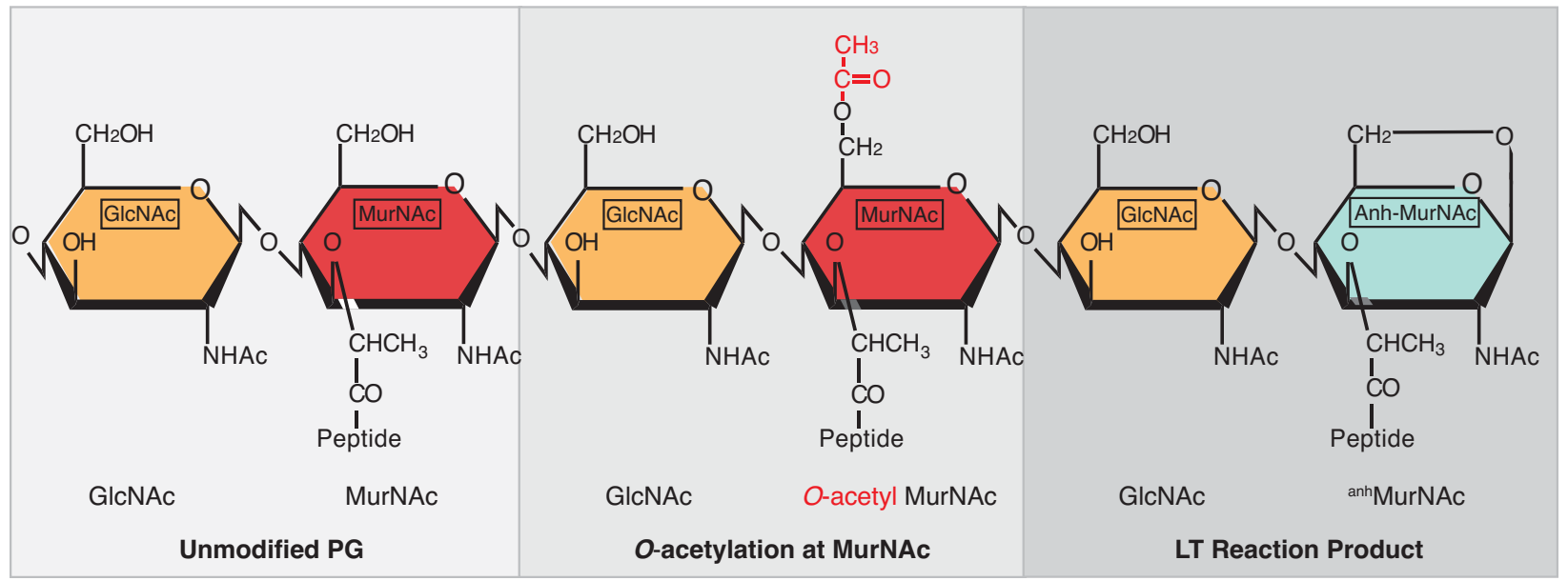

Figure 1.7. O-acetylation of MurNAc.

Depicted is a hypothetical hexa-saccharide made up of GlcNAc-1,4-MurNAc repeats showing the C-6 hydroxyl residues involved in O-acetylation and a terminal 1,6-anhydro-MurNAc cap formed by lytic transglycosylases. Adapted from Moynihan \& Clarke (2011) ${ }^{131}$ 


\subsection{DISSERTATION OVERVIEW}

In summary, lytic transglycosylases serve a variety of essential functions in the intricate metabolism of the cell wall meshwork. They are critical players during core cell wall processes, such as turnover, expansion and division, and also contribute to more specialized processes, including insertion of trans-envelope complexes ${ }^{14,28}$. The influence of LTs extends beyond the cell wall in the form of liberated anhydro-muropeptides that have the potential to act as chemical messengers or effectors. In this range of vital capacities, LTs must be carefully controlled and a number of examples of how some bacteria may achieve this regulation have been discussed. However, much remains to be learned about the physiological functions and regulation of lytic transglycosylases in bacteria.

In particular, it is not known how or why many bacteria terminate glycan chains with 1,6anhydro-MurNAc residues. As discussed previously, studies in E. coli have shown that the terminal anhydro cap is introduced during or shortly after nascent glycan chain polymerization, implicating a LT in this early processing step ${ }^{105,106}$. Given that it is not known how PBP glycosyltransferases terminate glycan strand polymerization, it has been proposed that an endoacting LT may participate in halting the polymerization process and releasing nascent glycan strands from the synthesis complex ${ }^{28}$. In this model, the balance of LT activity and PG synthesis would also play a crucial role in establishing the length of glycan strands within the cell wall, a feature that is believed to be important for bacterial morphology ${ }^{132,133}$. Previously studied lytic transglycosylases do not appear to be involved in generating anhydro glycan caps and/or terminating glycan strand polymerization. In the case of $E$. coli, up to six LTs ( $\Delta$ mltA-E $\Delta$ slt) can be deleted without a significant reduction in the percentage of terminal anhydros in the cell wall matrix ${ }^{33}$. Altogether this suggests that other lytic transglycosylases remain to be discovered. 
The extensive functional overlap of cell wall cleaving enzymes in E. coli has hindered discovery of new factors involved in peptidoglycan processing. With this rationale in mind, we devised a novel genetic screening approach to identify new cell wall modifying factors in E. coli. As described in Chapter 2, this screen led to the identification of MltG (yceG), a gene of previously unknown function ${ }^{57}$. We have shown that MltG represents a novel family of lytic transglycosylases broadly conserved in gram-negative and gram-positive bacteria. Unlike previously identified LTs in E. coli, MltG is an endo-enzyme localized to the inner membrane, putting it in a unique position to specifically engage with PG synthases and nascent PG material. Overall, our data supports a model in which MltG may fulfill the role of the originally proposed terminase of PG polymerization ${ }^{57}$. Knowledge of the function and regulation of this new PG hydrolase will provide a more complete picture of the process of cell wall biogenesis and generate new insights into how to best target this process for the development of novel antibiotics. 


\subsection{REFERENCES}

1. Fischbach, M. A. \& Walsh, C. T. Antibiotics for Emerging Pathogens. Science 325, 1089-1093 (2009).

2. Walsh, C. T. \& Wencewicz, T. A. Prospects for new antibiotics: a molecule-centered perspective. J. Antibiot. 67, 7-22 (2014).

3. McKenna, M. Antibiotic resistance: the last resort. Nature 499, 394-396 (2013).

4. Brown, E. D. \& Wright, G. D. Antibacterial drug discovery in the resistance era. Nature 529, 336-343 (2016).

5. Demain, A. L. \& Elander, R. P. The $\beta$-lactam antibiotics: past, present, and future. Antonie van Leeuwenhoek 75(1-2), 5-19 (1999).

6. Fleming, A. On the antibacterial action of cultures of a penicillium, with special reference to their use in the isolation of B. influenzae. 1929. Br. J. Exp. Pathol 10, 226236 (1929).

7. Taubes, G. The bacteria fight back. Science 321, 356-361 (2008).

8. Höltje, J. V. Growth of the stress-bearing and shape-maintaining murein sacculus of Escherichia coli. Microbiology and molecular biology reviews : MMBR 62, 181-203 (1998).

9. Silhavy, T. J., Kahne, D. \& Walker, S. The Bacterial Cell Envelope. Cold Spring Harb Perspect Biol 2(5), a000414 (2010).

10. Weiner, J. H. \& Li, L. Proteome of the Escherichia coli envelope and technological challenges in membrane proteome analysis. Biochimica et Biophysica Acta (BBA) Biomembranes 1778, 1698-1713 (2008).

11. Koch, A. L. Growth and form of the bacterial cell wall. American Scientist 78(4), 327341 (1990).

12. Young, K. D. The Selective Value of Bacterial Shape. Microbiology and Molecular Biology Reviews 70, 660-703 (2006).

13. Dijkstra, A. J. Ch 1: General Intro. Doctoral Dissertation, U.Groningen 1-34 (1997).

14. Vollmer, W. \& Bertsche, U. Murein (peptidoglycan) structure, architecture and biosynthesis in Escherichia coli. Biochimica et Biophysica Acta (BBA) - Biomembranes 1778, 1714-1734 (2008).

15. Typas, A., Banzhaf, M., Gross, C. A. \& Vollmer, W. From the regulation of peptidoglycan synthesis to bacterial growth and morphology. Nature reviews. Microbiology 10(2), 123-136 (2012). 
16. Sham, L. T. et al. MurJ is the flippase of lipid-linked precursors for peptidoglycan biogenesis. Science 345, 220-222 (2014).

17. Yang, D. C. The ABC's of Cell Division: Regulation of Peptidoglycan Amidase Activity during Cytokinesis in Escherichia coli. Doctoral Dissertation, Harvard University, 1169 (2012).

18. Cho, H., Uehara, T. \& Bernhardt, T. G. Beta-lactam antibiotics induce a lethal malfunctioning of the bacterial cell wall synthesis machinery. Cell 159, 1300-1311 (2014).

19. Sauvage, E., Kerff, F. D. R., Terrak, M., Ayala, J. A. \& Charlier, P. The penicillinbinding proteins: structure and role in peptidoglycan biosynthesis. FEMS microbiology reviews 32, 234-258 (2008).

20. Yousif, S. Y., Broome-Smith, J. K. \& Spratt, B. G. Lysis of Escherichia coli by betalactam antibiotics: deletion analysis of the role of penicillin-binding proteins $1 \mathrm{~A}$ and $1 \mathrm{~B}$. J. Gen. Microbiol. 131, 2839-2845 (1985).

21. Kato, J., Suzuki, H. \& Hirota, Y. Dispensability of either penicillin-binding protein-1a or $-1 \mathrm{~b}$ involved in the essential process for cell elongation in Escherichia coli. Molecular I\& general genetics : MGG 200, 272-277 (1985).

22. Paradis-Bleau, C. et al. Lipoprotein Cofactors Located in the Outer Membrane Activate Bacterial Cell Wall Polymerases. Cell 143, 1110-1120 (2010).

23. Di Berardino, M., Dijkstra, A., Stüber, D., Keck, W. \& Gubler, M. The monofunctional glycosyltransferase of Escherichia coli is a member of a new class of peptidoglycansynthesising enzymes. FEBS letters 392, 184-188 (1996).

24. Derouaux, A. et al. The monofunctional glycosyltransferase of Escherichia coli localizes to the cell division site and interacts with penicillin-binding protein $3, \mathrm{FtsW}$, and FtsN. Journal of bacteriology 190, 1831-1834 (2008).

25. Spratt, B. G. Distinct penicillin binding proteins involved in the division, elongation, and shape of Escherichia coli K12. PNAS 72, 2999-3003 (1975).

26. Vollmer, W. Morphogenesis of Escherichia coli. Current opinion in microbiology 4, 625-633 (2001).

27. Blaauwen, den, T., de Pedro, M. A., Nguyen-Distèche, M. \& Ayala, J. A. Morphogenesis of rod-shaped sacculi. FEMS microbiology reviews 32, 321-344 (2008).

28. van Heijenoort, J. Peptidoglycan Hydrolases of Escherichia coli. Microbiology and Molecular Biology Reviews 75, 636-663 (2011).

29. Höltje, J. V., Mirelman, D., Sharon, N. \& Schwarz, U. Novel type of murein transglycosylase in Escherichia coli. Journal of bacteriology 124, 1067-1076 (1975). 
30. Thunnissen, A.-M. W. H., Rozeboom, H. J., Kalk, K. H. \& Dijkstra, B. W. Structure of the 70-kDa Soluble Lytic Transglycosylase Complexed with Bulgecin A. Implications for the Enzymic Mechanism. Biochemistry 34, 12729-12737 (1995).

31. Thunnissen, A.-M. W. H., Isaacs, N. W. \& Dijkstral, B. W. The catalytic domain of a bacterial lytic transglycosylase defines a novel class of lysozymes. Proteins 22, 245-258 (1995).

32. Heidrich, C. et al. Involvement of N-acetylmuramyl-L-alanine amidases in cell separation and antibiotic-induced autolysis of Escherichia coli. Mol. Microbiol. 41, 167178 (2001).

33. Heidrich, C., Ursinus, A., Berger, J., Schwarz, H. \& Höltje, J. V. Effects of Multiple Deletions of Murein Hydrolases on Viability, Septum Cleavage, and Sensitivity to Large Toxic Molecules in Escherichia coli. Journal of bacteriology 184, 6093-6099 (2002).

34. Priyadarshini, R., de Pedro, M. A. \& Young, K. D. Role of peptidoglycan amidases in the development and morphology of the division septum in Escherichia coli. Journal of bacteriology 189, 5334-5347 (2007).

35. Singh, S. K., SaiSree, L., Amrutha, R. N. \& Reddy, M. Three redundant murein endopeptidases catalyse an essential cleavage step in peptidoglycan synthesis of Escherichia coli K12. Mol. Microbiol. 86, 1036-1051 (2012).

36. Singh, S. K., Parveen, S., SaiSree, L. \& Reddy, M. Regulated proteolysis of a crosslink-specific peptidoglycan hydrolase contributes to bacterial morphogenesis. PNAS 112(35), 10956-10961 (2015).

37. Engel, H., Smink, A. J., van Wijngaarden, L. \& Keck, W. Murein-metabolizing enzymes from Escherichia coli: existence of a second lytic transglycosylase. Journal of bacteriology 174, 6394-6403 (1992).

38. Templin, M. F., Edwards, D. H. \& Höltje, J. V. A murein hydrolase is the specific target of bulgecin in Escherichia coli. J. Biol. Chem. 267, 20039-20043 (1992)

39. Lommatzsch, J., Templin, M. F., Kraft, A. R., Vollmer, W. \& al, E. Outer membrane localization of murein hydrolases: MltA, a third lipoprotein lytic transglycosylase in Escherichia coli. Journal of bacteriology 179, 5465-5470 (1997).

40. Ehlert, K., Höitje, J.-V. \& Templin, M. F. Cloning and expression of a murein hydrolase lipoprotein from Escherichia coli. Mol. Microbiol. 16, 761-768 (1995).

41. DIJKSTRA, A. J. \& KECK, W. Identification of New Members of the Lytic Transglycosylase Family in Haemophilus influenzaeand Escherichia coli. Microbial Drug Resistance 2, 141-145 (1996).

42. The lytic transglycosylase family of Escherichia coli: in vitro activity versus in vivo function. Doctoral Dissertation, University of Groningen, (1997). 
43. Kraft, A., Templin, M. \& ltje, J. H. O. Membrane-bound lytic endotransglycosylase in Escherichia coli. Journal of bacteriology 180, 3441-3447 (1998).

44. Scheurwater, E. M. \& Clarke, A. J. The C-terminal Domain of Escherichia coli Y fhD Functions as a Lytic Transglycosylase. Journal of Biological Chemistry 283, 8363-8373 (2008).

45. Korsak, D., Liebscher, S. \& Vollmer, W. Susceptibility to Antibiotics and -Lactamase Induction in Murein Hydrolase Mutants of Escherichia coli. Antimicrobial agents and chemotherapy 49, 1404-1409 (2005).

46. Scheurwater, E., Reid, C. W. \& Clarke, A. J. Lytic transglycosylases: Bacterial spacemaking autolysins. The international journal of biochemistry \& cell biology 40, 586-591 (2008).

47. Reid, C. W., Blackburn, N. T., Legaree, B. A., Auzanneau, F.-I. \& Clarke, A. J. Inhibition of membrane-bound lytic transglycosylase B by NAG-thiazoline. FEBS letters 574, 73-79 (2004).

48. Beachey, E. H., Keck, W., de Pedro, M. A. \& Schwarz, U. Exoenzymatic activity of transglycosylase isolated from Escherichia coli. European journal of biochemistry / FEBS 116, 355-358 (1981).

49. Hartmann, R., Höltje, J. V. \& Schwarz, U. Targets of penicillin action in Escherichia coli. Nature 235, 426-429 (1972).

50. Romeis, T., Vollmer, W. \& HÃ ltje, J.-V. Characterization of three different lytic transglycosylases in Escherichia coli. FEMS microbiology letters 111, 141-146 (1993).

51. Lee, M. et al. Reactions of All Escherichia coliLytic Transglycosylases with Bacterial Cell Wall. Journal of the American Chemical Society 135, 3311-3314 (2013).

52. Thunnissen, A., Dijkstra, A. J. \& Kalk, K. H. Doughnut-shaped structure of a bacterial muramidase revealed by X-ray crystallography. Nature 367 (6465), 750-753 (1994).

53. Artola-Recolons, C. et al. Structure and cell wall cleavage by modular lytic transglycosylase MltC of Escherichia coli. ACS Chem. Biol. 9, 2058-2066 (2014).

54. Artola-Recolons, C. et al. High-Resolution Crystal Structure of MltE, an Outer Membrane-Anchored Endolytic Peptidoglycan Lytic Transglycosylase from Escherichia coli. Biochemistry 50, 2384-2386 (2011).

55. van Asselt, E. J., Thunnissen, A.-M. W. H. \& Dijkstra, B. W. High resolution crystal structures of the Escherichia coli lytic transglycosylase slt70 and its complex with a peptidoglycan fragment. Journal of molecular biology 291, 877-898 (1999).

56. Ursinus, A. \& Höltje, J. V. Purification and properties of a membrane-bound lytic transglycosylase from Escherichia coli. Journal of bacteriology 176, 338-343 (1994). 
57. Yunck, R., Cho, H. \& Bernhardt, T. G. Identification of MltG as a potential terminase for peptidoglycan polymerization in bacteria. Mol. Microbiol. 99(4), 700-718 (2015).

58. Jorgenson, M. A., Chen, Y., Yahashiri, A., Popham, D. L. \& Weiss, D. S. The bacterial septal ring protein RlpA is a lytic transglycosylase that contributes to rod shape and daughter cell separation in Pseudomonas aeruginosa. Mol. Microbiol. 93, 113-128 (2014).

59. Blackburn, N. T. \& Clarke, A. J. Identification of four families of peptidoglycan lytic transglycosylases. J. Mol. Evol. 52, 78-84 (2001).

60. Van Straaten, K. E., Dijkstra, B. W., Vollmer, W. \& Thunnissen, A.-M. W. H. Crystal Structure of MltA from Escherichia coli Reveals a Unique Lytic Transglycosylase Fold. Journal of molecular biology 352, 1068-1080 (2005).

61. Jacobs, C., Huang, L. J., Bartowsky, E., Normark, S. \& Park, J. T. Bacterial cell wall recycling provides cytosolic muropeptides as effectors for beta-lactamase induction. The EMBO journal 13, 4684-4694 (1994).

62. Kraft, A. R., Prabhu, J., Ursinus, A. \& Höltje, J.-V. Interference with murein turnover has no effect on growth but reduces beta-lactamase induction in Escherichia coli. 181, 7192-7198 (1999).

63. Goodell, E. W. \& Schwarz, U. Release of cell wall peptides into culture medium by exponentially growing Escherichia coli. Journal of bacteriology 162(1), 391-397 (1985).

64. Reith, J. \& Mayer, C. Peptidoglycan turnover and recycling in Gram-positive bacteria. Applied microbiology and biotechnology 92, 1-11 (2011).

65. Park, J. T. \& Uehara, T. How Bacteria Consume Their Own Exoskeletons (Turnover and Recycling of Cell Wall Peptidoglycan). Microbiology and Molecular Biology Reviews 72, 211-227 (2008).

66. Cheng, Q. \& Park, J. T. Substrate Specificity of the AmpG Permease Required for Recycling of Cell Wall Anhydro-Muropeptides. Journal of bacteriology 184, 6434-6436 (2002).

67. Cheng, Q., Li, H., Merdek, K. \& Park, J. T. Molecular characterization of the beta-Nacetylglucosaminidase of Escherichia coli and its role in cell wall recycling. Journal of bacteriology 182, 4836-4840 (2000).

68. Vötsch, W. \& Templin, M. F. Characterization of a $\beta$-N-acetylglucosaminidase ofEscherichia coli and Elucidation of Its Role in Muropeptide Recycling and $\beta$ Lactamase Induction. Journal of Biological Chemistry 275, 39032-39038 (2000).

69. Jacobs, C., Joris, B., Jamin, M. \& Klarsov, K. AmpD, essential for both $\beta$-lactamase regulation and cell wall recycling, is a novel cytosolic N-acetylmuramyl-L-alanine amidase. Molecular Microbiology 15, 553-559 (1995). 
70. Johnson, J. W., Fisher, J. F. \& Mobashery, S. Bacterial cell-wall recycling. Annals of the New York Academy of Sciences 1277, 54-75 (2012).

71. Fisher, J. F. \& Mobashery, S. The sentinel role of peptidoglycan recycling in the $\beta$ lactam resistance of the Gram-negative Enterobacteriaceae and Pseudomonas aeruginosa. Bioorg. Chem. 56, 41-48 (2014).

72. Templin, M. F. A defect in cell wall recycling triggers autolysis during the stationary growth phase of Escherichia coli. The EMBO journal 18, 4108-4117 (1999).

73. Lindberg, F., Lindquist, S. \& Normark, S. Inactivation of the ampD gene causes semiconstitutive overproduction of the inducible Citrobacter freundii beta-lactamase. Journal of bacteriology 169, 1923-1928 (1987).

74. Jacobs, C., Frère, J. M. \& Normark, S. Cytosolic intermediates for cell wall biosynthesis and degradation control inducible beta-lactam resistance in gram-negative bacteria. Cell 88, 823-832 (1997).

75. Normark, S. beta-Lactamase induction in gram-negative bacteria is intimately linked to peptidoglycan recycling. Microbial drug resistance 1, 111-114 (1995).

76. Vadlamani, G. et al. The $\beta$-lactamase gene regulator AmpR is a tetramer that recognizes and binds the D-Ala-D-Ala motif of its repressor UDP-N-acetylmuramic acid (MurNAc)-pentapeptide. Journal of Biological Chemistry 290, 2630-2643 (2015).

77. Boneca, I. The role of peptidoglycan in pathogenesis. Current opinion in microbiology 8, 46-53 (2005).

78. Cloud-Hansen, K. A. et al. Breaching the great wall: peptidoglycan and microbial interactions. Nature reviews. Microbiology 4, 710-716 (2006).

79. Dokter, W. H., Dijkstra, A. J., Koopmans, S. B. \& Stulp, B. K. G (Anh) MTetra, a natural bacterial cell wall breakdown product, induces interleukin-1 beta and interleukin6 expression in human monocytes. mechanisms involved in inflammatory cytokine expression. Journal of Biological Chemistry 269, 4201-4206 (1994).

80. Cloud-Hansen, K. A., Hackett, K. T., Garcia, D. L. \& Dillard, J. P. Neisseria gonorrhoeae Uses Two Lytic Transglycosylases To Produce Cytotoxic Peptidoglycan Monomers. Journal of bacteriology 190, 5989-5994 (2008).

81. Oh, H. S., Kvitko, B. H., Morello, J. E. \& Collmer, A. Pseudomonas syringae Lytic Transglycosylases Coregulated with the Type III Secretion System Contribute to the Translocation of Effector Proteins into Plant Cells. Journal of bacteriology 189, 82778289 (2007).

82. Scheurwater, E. M. \& Burrows, L. L. Maintaining network security: how macromolecular structures cross the peptidoglycan layer. FEMS microbiology letters 318, 1-9 (2011). 
83. Höltje, J. V. A hypothetical holoenzyme involved in the replication of the murein sacculus of Escherichia coli. Microbiology 142(8), 1911-1918 (1996).

84. Gerding, M. A. et al. Self-Enhanced Accumulation of FtsN at Division Sites and Roles for Other Proteins with a SPOR Domain (DamX, DedD, and RlpA) in Escherichia coli Cell Constriction. Journal of bacteriology 191, 7383-7401 (2009).

85. Arends, S. J. R. et al. Discovery and Characterization of Three New Escherichia coli Septal Ring Proteins That Contain a SPOR Domain: DamX, DedD, and RlpA. Journal of bacteriology 192, 242-255 (2009).

86. Powell, A. J., Liu, Z.-J., Nicholas, R. A. \& Davies, C. Crystal Structures of the Lytic Transglycosylase MltA from N.gonorrhoeae and E.coli: Insights into Interdomain Movements and Substrate Binding. Journal of molecular biology 359, 122-136 (2006).

87. Adu-Bobie, J. et al. GNA33 of Neisseria meningitidis is a lipoprotein required for cell separation, membrane architecture, and virulence. Infection and immunity 72, 1914 1919 (2004).

88. Höltje, J. V. 'Three for one'- a Simple Growth Mechanism that Guarantees a Precise Copy of the Thin, Rod-Shaped Murein Sacculus of Escherichia coli. Bacterial Growth and Lysis (Springer US), 419-426 (1993).

89. Koch, A. L. The three-for-one model for Gram-negative wall growth: a problem and a possible solution. FEMS microbiology letters 162, 127-134 (1998).

90. Burman, L. G. \& Park, J. T. Molecular model for elongation of the murein sacculus of Escherichia coli. PNAS 81, 1844-1848 (1984).

91. Park, J. T. \& Burman, L. G. Elongation of the murein sacculus of Escherichia coli. Ann. Inst. Pasteur Microbiol. 136A, 51-58 (1985).

92. Vollmer, W., Rechenberg, von, M. \& Höltje, J. V. Demonstration of Molecular Interactions between the Murein Polymerase PBP1B, the Lytic Transglycosylase MltA, and the Scaffolding Protein MipA of Escherichia coli. Journal of Biological Chemistry 274, 6726-6734 (1999)

93. Romeis, T. \& ltje, J. H. O. Specific interaction of penicillin-binding proteins 3 and 7/8 with soluble lytic transglycosylase in Escherichia coli. J. Biol. Chem. 269, 21603-21607 (1994).

94. Alaedini, A. \& Day, R. A. Identification of two penicillin-binding multienzyme complexes in Haemophilus influenzae. Biochemical and biophysical research communications 264, 191-195 (1999).

95. Jennings, G. T. et al. GNA33 from Neisseria meningitidisserogroup B encodes a membrane-bound lytic transglycosylase (MltA). Eur J Biochem 269, 3722-3731 (2002). 
96. Legaree, B. A. \& Clarke, A. J. Interaction of Penicillin-Binding Protein 2 with Soluble Lytic Transglycosylase B1 in Pseudomonas aeruginosa. Journal of bacteriology 190, 6922-6926 (2008).

97. Hett, E. C., Chao, M. C. \& Rubin, E. J. Interaction and Modulation of Two Antagonistic Cell Wall Enzymes of Mycobacteria. PLoS pathogens 6, e1001020 (2010).

98. Zapun, A., Noirclerc-Savoye, M., Helassa, N. \& Vernet, T. Peptidoglycan Assembly Machines: The Biochemical Evidence. Microbial drug resistance 18, 256-260 (2012).

99. Rechenberg, von, M., Ursinus, A. \& Höltje, J. V. Affinity chromatography as a means to study multienzyme complexes involved in murein synthesis. Microbial drug resistance (Larchmont, N.Y.) 2, 155-157 (1996).

100. Müller, P. et al. The essential cell division protein FtsN interacts with the murein (peptidoglycan) synthase PBP1B in Escherichia coli. J. Biol. Chem. 282, 36394-36402 (2007).

101. Sung, M.-T. et al. Crystal structure of the membrane-bound bifunctional transglycosylase PBP1b from Escherichia coli. PNAS 106, 8824-8829 (2009).

102. Bertsche, U., Breukink, E., Kast, T. \& Vollmer, W. In vitro murein peptidoglycan synthesis by dimers of the bifunctional transglycosylase-transpeptidase PBP1B from Escherichia coli. J. Biol. Chem. 280, 38096-38101 (2005).

103. Glauner, B., Höltje, J. V. \& Schwarz, U. The composition of the murein of Escherichia coli. J. Biol. Chem. 263, 10088-10095 (1988).

104. Glauner, B. Separation and quantification of muropeptides with high-performance liquid chromatography. Anal. Biochem. 172, 451-464 (1988).

105. Glauner, B. \& al, E. Growth pattern of the murein sacculus of Escherichia coli. J. Biol. Chem. 265, 18988-18996 (1990).

106. Burman, L. G. \& Park, J. T. Changes in the composition of Escherichia coli murein as it ages during exponential growth. Journal of bacteriology 155, 447-453 (1983).

107. Zahrl, D. Peptidoglycan degradation by specialized lytic transglycosylases associated with type III and type IV secretion systems. Microbiology 151, 3455-3467 (2005).

108. Koraimann, G. Lytic transglycosylases in macromolecular transport systems of Gramnegative bacteria. Cellular and molecular life sciences : CMLS 60, 2371-2388 (2003).

109. Demchick, P. \& Koch, A. L. The permeability of the wall fabric of Escherichia coli and Bacillus subtilis. Journal of bacteriology 178, 768-773 (1996). 
110. Vázquez-Laslop, N., Lee, H., Hu, R. \& Neyfakh, A. A. Molecular sieve mechanism of selective release of cytoplasmic proteins by osmotically shocked Escherichia coli. Journal of bacteriology 183, 2399-2404 (2001).

111. Vollmer, W., Joris, B., Charlier, P. \& Foster, S. Bacterial peptidoglycan (murein) hydrolases. FEMS microbiology reviews 32, 259-286 (2008).

112. Kohler, P. L., Hamilton, H. L., Cloud-Hansen, K. \& Dillard, J. P. AtlA Functions as a Peptidoglycan Lytic Transglycosylase in the Neisseria gonorrhoeae Type IV Secretion System. Journal of bacteriology 189, 5421-5428 (2007).

113. Roure, S. et al. Peptidoglycan maturation enzymes affect flagellar functionality in bacteria. Mol. Microbiol. 86, 845-856 (2012).

114. Betzner, A. S., Ferreira, L. C. S., Höltje, J. V. \& Keck, W. Control of the activity of the soluble lytic transglycosylase by the stringent response in Escherichia coli. FEMS microbiology letters 67, 161-164 (2006).

115. Baron, C., Llosa, M., Zhou, S. \& Zambryski, P. C. VirB1, a component of the Tcomplex transfer machinery of Agrobacterium tumefaciens, is processed to a C-terminal secreted product, VirB1. Journal of bacteriology 179, 1203-1210 (1997).

116. Höppner, C., Liu, Z., Domke, N., Binns, A. N. \& Baron, C. VirB1 orthologs from Brucella suis and pKM101 complement defects of the lytic transglycosylase required for efficient type IV secretion from Agrobacterium tumefaciens. Journal of bacteriology 186, 1415-1422 (2004).

117. Viollier, P. H. \& Shapiro, L. A lytic transglycosylase homologue, PleA, is required for the assembly of pili and the flagellum at the Caulobacter crescentus cell pole. Mol. Microbiol. 49, 331-345 (2003).

118. Errington, J. Regulation of endospore formation in Bacillus subtilis. Nature reviews. Microbiology 1, 117-126 (2003).

119. Chao, M. C. et al. Protein complexes and proteolytic activation of the cell wall hydrolase RipA regulate septal resolution in mycobacteria. PLoS pathogens 9, e1003197 (2013).

120. Morlot, C., Uehara, T., Marquis, K. A., Bernhardt, T. G. \& Rudner, D. Z. A highly coordinated cell wall degradation machine governs spore morphogenesis in Bacillus subtilis. Genes |\& development 24, 411-422 (2010).

121. Divakaruni, A. V., Baida, C., White, C. L. \& Gober, J. W. The cell shape proteins MreB and $\mathrm{MreC}$ control cell morphogenesis by positioning cell wall synthetic complexes. Mol. Microbiol. 66, 174-188 (2007).

122. Divakaruni, A. V., Loo, R. R. O., Xie, Y., Loo, J. A. \& Gober, J. W. The cell-shape protein $\mathrm{MreC}$ interacts with extracytoplasmic proteins including cell wall assembly complexes in Caulobacter crescentus. PNAS 102, 18602-18607 (2005). 
123. Ward, D. V., Draper, O., Zupan, J. R. \& Zambryski, P. C. Peptide linkage mapping of the Agrobacterium tumefaciens vir-encoded type IV secretion system reveals protein subassemblies. PNAS 99, 11493-11500 (2002).

124. Uehara, T., Dinh, T. \& Bernhardt, T. G. LytM-Domain Factors Are Required for Daughter Cell Separation and Rapid Ampicillin-Induced Lysis in Escherichia coli. Journal of bacteriology 191, 5094-5107 (2009).

125. Uehara, T., Parzych, K. R., Dinh, T. \& Bernhardt, T. G. Daughter cell separation is controlled by cytokinetic ring-activated cell wall hydrolysis. The EMBO journal 29, $1412-1422(2010)$.

126. Peters, N. T., Dinh, T. \& Bernhardt, T. G. A Fail-Safe Mechanism in the Septal Ring Assembly Pathway Generated by the Sequential Recruitment of Cell Separation Amidases and Their Activators. Journal of bacteriology 193, 4973-4983 (2011).

127. Yahashiri, A., Jorgenson, M. A. \& Weiss, D. S. Bacterial SPOR domains are recruited to septal peptidoglycan by binding to glycan strands that lack stem peptides. PNAS 112, 11347-11352 (2015).

128. Deckers, D. et al. Periplasmic lysozyme inhibitor contributes to lysozyme resistance in Escherichia coli. Cellular and molecular life sciences : CMLS 61, 1229-1237 (2004).

129. Clarke, C. A., Scheurwater, E. M. \& Clarke, A. J. The Vertebrate Lysozyme Inhibitor Ivy Functions to Inhibit the Activity of Lytic Transglycosylase. Journal of Biological Chemistry 285, 14843-14847 (2010).

130. Control of lytic transglycosylase activity within bacterial cell walls. Caister Academic press, Norfolk, UK (2012).

131. Moynihan, P. J. \& Clarke, A. J. O-Acetylated peptidoglycan: Controlling the activity of bacterial autolysins and lytic enzymes of innate immune systems. The international journal of biochemistry \& cell biology 43, 1655-1659 (2011).

132. Harz, H., Burgdorf, K. \& Höltje, J.-V. Isolation and separation of the glycan strands from murein of Escherichia coli by reversed-phase high-performance liquid chromatography. Anal. Biochem. 190, 120-128 (1990).

133. Furchtgott, L., Wingreen, N. S. \& Huang, K. C. Mechanisms for maintaining cell shape in rod-shaped Gram-negative bacteria. Mol. Microbiol. 81, 340-353 (2011). 
Chapter 2. Identification of MltG as a Potential Terminase for Peptidoglycan Polymerization in Bacteria 


\section{ATTRIBUTIONS}

The work presented in this chapter was performed primarily be Rachel Yunck. Hongbaek Cho performed turnover experiments to help explore the role of MltG in nascent PG synthesis and generated Figure 2.14. All remaining experiments and figures/tables were performed and generated by Rachel Yunck. The manuscript was written by Rachel Yunck and Thomas Bernhardt.

The work in this chapter is reprinted with permission from John Wiley \& Sons Inc: Yunck, R., Cho, H. \& Bernhardt, T. G. Identification of MltG as a potential terminase for peptidoglycan polymerization in bacteria. Mol. Microbiol. 99, 700-718 (2015). 


\title{
Chapter 2: Identification of MItG as a potential terminase for peptidoglycan polymerization in bacteria
}

\author{
Rachel Yunck ${ }^{1}$, Hongbaek Cho ${ }^{1}$, and Thomas G. Bernhardt ${ }^{1}$ \\ ${ }^{1}$ Department of Microbiology and Immunobiology, Harvard Medical School, Boston, MA 02115
}

\subsection{INTRODUCTION}

A key component of the bacterial cell envelope is the cell wall matrix, an essential structure that fortifies the cytoplasmic membrane against osmotic rupture and confers cell shape $^{1}$. The cell wall is made up of a meshwork of peptidoglycan (PG), a unique bacterial heteropolymer constructed from long glycan chains of $\beta$-1-4-linked $\mathrm{N}$-acetylmuramic acid (MurNAc) and N-acetylglucosamine (GlcNAc) sugars connected by short stem-peptides to form a net-like macromolecule that surrounds the cell. In E. coli and other gram-negative bacteria, the cell wall exists as a thin matrix layer sandwiched between an inner and outer membrane ${ }^{2}$. In contrast, gram-positive bacteria possess a thick layer of PG that surrounds the inner membrane and is exposed to the environment ${ }^{2}$. Any perturbations to the process of PG biogenesis can lead to a catastrophic breach in the cell wall and ultimately cause cell lysis. This sequence of events is exploited by many antibiotics used clinically to treat infections, including penicillin and vancomycin. Unfortunately, resistance to these and other drugs is gradually eroding the effectiveness of our antibiotic arsenal ${ }^{3,4}$. New treatments capable of overcoming the spread of resistance are therefore sorely needed. An attractive avenue towards the development of such therapies is the identification of additional weak points in the cell wall biogenesis pathway amenable to antibiotic targeting. These novel vulnerabilities are most likely to be discovered through a greater understanding of the fundamental mechanisms underlying the process. 
To build the PG layer, synthases called high-molecular weight penicillin-binding proteins (PBPs) are required ${ }^{5}$. These synthases are the primary targets of penicillin and related betalactam antibiotics ${ }^{6}$. They are subdivided into class A (aPBPs) and class B (bPBPs) enzymes. aPBPs are bifunctional and possess both glycosyltransferase (GT) activity for polymerizing the glycan strands and transpeptidase (TP) activity for crosslinking them. bPBPs, on the other hand, are only known to possess TP activity. In addition to the PG synthases, enzymes capable of cleaving bonds in the cell wall network known as PG hydrolases or autolysins are also important for proper PG assembly ${ }^{7}$. Several autolysins have recently been implicated in the expansion of the cell wall matrix during growth ${ }^{8-12}$. They are thought to break bonds in the existing structure to allow for the insertion of new material. Enzymes with PG hydrolase activity are also known to be required for cleaving the shared cell wall septum formed during cytokinesis to promote daughter cell separation ${ }^{13,7}$. Outside of the handful of autolysins connected with these morphogenic processes, relatively few of the many predicted PG cleaving enzymes encoded by bacteria have a clearly defined physiological function. For example, Escherichia coli encodes seven lytic transglycosylases (LTs) (Slt, MltA-F) capable of cleaving the glycan strands of PG ${ }^{14}$. It also encodes RlpA, which has been shown to have LT activity in Pseudomonas aeruginosa and likely has the same enzymatic function in $E$. coli ${ }^{15}$. Besides Slt and RlpA, which have recently been implicated in PG quality control ${ }^{16}$ and cell division ${ }^{15}$, respectively, little is known about the functions of the other LT enzymes other than their involvement in general PG turnover, a minor role in daughter cell separation, and potential function in the assembly of trans-envelope structures ${ }^{17-19,14}$. 

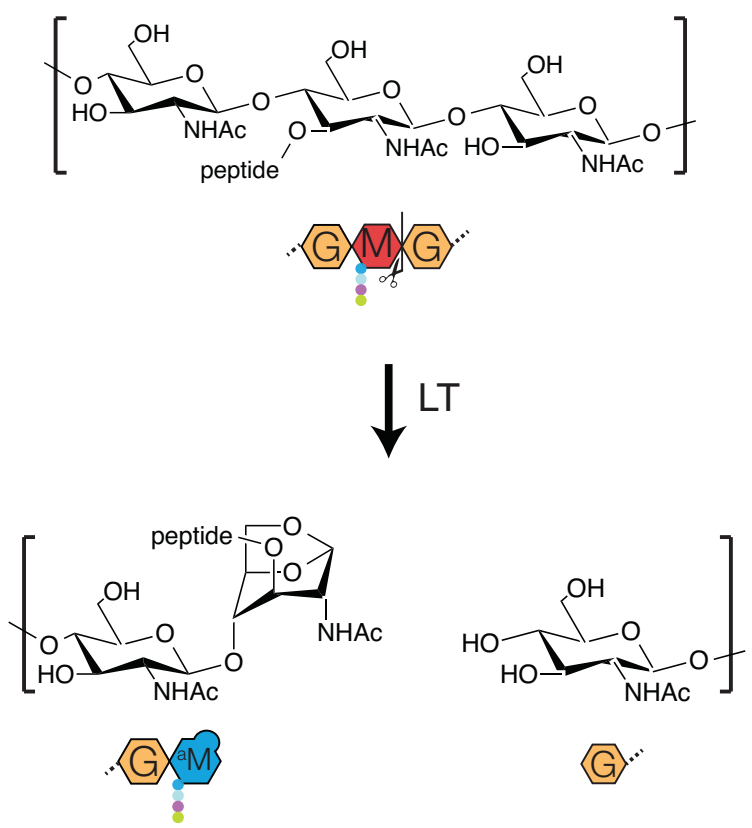

\section{Figure 2.1. Lytic transglycosylase reaction.}

Diagram showing the lytic transglycosylase reaction. Strand cleavage results in the formation of an anhMurNAc sugar at what would normally be the reducing end of the polysaccharide. Shown below the chemical structures are cartoon representations of the substrate and products.

Rather than simply hydrolyzing glycan strands, LTs catalyze an intramolecular transglycosylation reaction that forms an anhydro ring between the $\mathrm{C} 1$ and $\mathrm{C} 6$ atoms of MurNAc (anhMurNAc) coincident with strand breakage ${ }^{14}$ (Figure 2.1). Most, if not all, glycan strands in

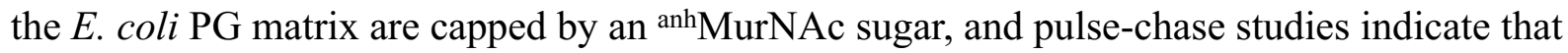
this cap is formed shortly after synthesis ${ }^{20,21,1}$. These observations have long suggested that one or more LT enzymes may function in the processing of nascent PG to terminate strand synthesis. However, the anhMurNAc content of the PG matrix changed surprisingly little in a mutant lacking six LTs (Slt, MltA-E) ${ }^{22}$. This observation has led to the suggestion that either other LT enzymes may be responsible for forming the anh MurNAc caps in the PG matrix, or that the 
structure is formed by the polymerase enzymes through an as yet uncharacterized termination $\operatorname{activity}^{23,22}$.

Because of their status as major drug targets, the structures and biochemical activities of the PBPs have been extensively characterized in vitro ${ }^{5}$. However, it remains poorly understood how these proteins are regulated in vivo and what factors coordinate their activity with the PG autolysins that remodel the matrix. We therefore initiated a genetic screen designed to identify regulators of the E. coli $\mathrm{aPBPs}^{24}$. The screen was carried out several years ago and was based on the synthetic lethal phenotype of mutants lacking both of the major aPBPs, PBP1a and PBP1b. We reasoned that a screen for mutations synthetically lethal with PBP1b inactivation would yield mutations in the gene for PBP1a plus mutations in any gene coding for a factor required for PBP1a function. Similarly, the complementary screen for mutations synthetically lethal with PBP1a inactivation should identify factors required for PBP1b activity. Armed with this logic and a plasmid-loss genetic assay, we successfully identified the outer membrane lipoproteins LpoA and LpoB as critical activators of PBP1a and PBP1b, respectively ${ }^{24,} 25$.

In this report, we revisit the screen for mutants synthetically lethal with PBP1b. However, instead of using transposon mutagenesis, we searched for genes inducing a lethal phenotype in PBP1b-defective cells when they are overexpressed from a multicopy plasmid. We reasoned that such a screen might identify negative regulators of PBP1a activity or factors causing damage to the PG network that is poorly tolerated when PG biogenesis is crippled by PBP1b inactivation. Multicopy plasmids containing the $y c e G$ gene were found to cause a severe growth defect in the absence of PBP1b. Biochemical studies indicated that YceG is a new LT enzyme with endoglycosidase activity capable of cleaving PG at internal positions along the length of glycan strands. Moreover, we found that YceG is a transmembrane protein anchored in the inner 
membrane and thus has a distinct subcellular localization from the other LT enzymes encoded by E. coli, which are either periplasmic (Slt) or outer membrane-anchored (MltA, B, C, D, E, F and RlpA) ${ }^{14,15}$. We therefore renamed this $\mathrm{LT}$ as MltG for membrane lytic transglycosylase $\underline{\mathrm{G}}$. Although loss of MltG function was not lethal or associated with a detectable morphological phenotype, its inactivation led to a significant increase in glycan strand length in the mature PG matrix. Additionally, bacterial two-hybrid analysis indicated that MltG forms a complex with PBP1b, and radiolabeling studies detected MltG-dependent processing of nascent PG in vivo. Our combined results are, therefore, consistent with a model in which MltG associates with PG synthetic complexes to cleave nascent polymers and terminate their elongation. Given its extremely broad conservation relative to other LT enzymes, we suspect that MltG performs a similar function in a wide range of bacteria. Consistent with this possibility, work in the Winkler laboratory currently in preparation for publication indicates that $\mathrm{MltG}$ is an essential cell wall assembly factor in Streptococcus pneumoniae (M. Winkler, personal communication).

\subsection{RESULTS}

\section{A multicopy lethal screen for new cell wall biogenesis factors}

Although mutants lacking the cell wall synthase PBP1b are viable, they lyse at an elevated frequency ${ }^{26}$ and are hypersensitive to beta-lactam antibiotics ${ }^{27}$, indicating they have an attenuated capacity for PG synthesis. In such mutants, PBP1a and its activator LpoA become essential $^{24,25}$. We therefore reasoned that a screen for genes rendering PBP1b essential when they are overexpressed from a multicopy plasmid might identify previously uncharacterized inhibitors of PBP1a/LpoA or novel PG-cleaving enzymes that damage the PG matrix to an extent that is not tolerated when cell wall synthesis is compromised by PBP1b inactivation. 
To screen for genes causing a conditional, multicopy lethal phenotype, we adapted the plasmid-loss assay used previously to identify synthetic lethal mutant combinations ${ }^{28,29,24}$. Strain TU122 $[\Delta p o n B]$ was transformed with the unstable mini-F vector pDY1, encoding ponB and a lacZ reporter under the control of an IPTG-inducible lactose promoter (Plac). TU122/pDY1 was then transformed with a multicopy plasmid library prepared by inserting random (1-4 kb) genomic fragments of E. coli MG1655 [WT] downstream of the arabinose promoter (Para) in a derivative of pBAD $33^{30}$. The resulting transformants were then plated on LB medium containing IPTG and X-gal, but without antibiotic selection for pDY1. Roughly 30,000 colonies were screened in the presence of arabinose and 20,000 in its absence. The vast majority of colonies formed under these conditions were either solid-white or sectored-blue, indicating that PBP1b was not essential and the pDY1 [Plac::ponB lacZ] plasmid could easily be lost (Figure 2.2 A-C). However, rare solid-blue colonies were also formed on media with or without arabinose supplementation (Figure 2.2A and Figure 2.2D), suggesting that these cells harbored a multicopy clone rendering PBP1b essential such that pDY1 could not be lost. A subset of these isolates (1/6 without and 5/6 with arabinose) became IPTG-dependent for growth, confirming their reliance on ponB expression from pDY1 for viability. The multicopy plasmids were isolated from these strains and sequenced to identify the inserted genomic region.

Plasmids containing a variety of inserts were identified in the screen, which was unlikely to be saturated. The isolate we focused on for this study encoded the gene of unknown function $y c e G$ (Figure 2.2E). The $y c e G$ has been implicated as potentially playing a role in cell envelope biogenesis in high-throughput studies ${ }^{31,32}$. Inactivation of $y c e G$ leads to a mild hypersensitivity to the beta-lactam mecillinam ${ }^{32}$, which we have confirmed (data not shown), and has been reported to suppress the phenotypes of deletions in some PG hydrolase encoding genes like 
$y e b A^{31}$. The significance of the latter result is unclear given that growth phenotypes for single mutants of $y e b A$ were not reported or observed in more focused studies of its function ${ }^{33,10}$. Based on the results presented below we have renamed the $y c e G$ gene $m l t G$ (membrane lytic transglycosylase $\underline{\mathrm{G}})$.

To confirm that $m l t G$ overexpression was responsible for rendering PBP1b essential in the screen isolate, we constructed an expression vector pRY53 producing $m l t G$ under Para control and transformed it into WT and $\triangle p o n B$ cells. WT cells harboring pRY53 were viable on solid agar with or without arabinose supplementation and grew to saturation in liquid M9-arabinose medium (Figure 2.2 F-G). Conversely, plating efficiency of $\Delta p o n B$ cells containing pRY53 was severely compromised on arabinose agar, and these cells became extremely misshapen and lysed following plasmid induction (Figure 2.2 F-I). We conclude that MltG overproduction renders PBP1b essential and that this phenotype is likely due to the induction of a severe defect in PG biogenesis. 
A
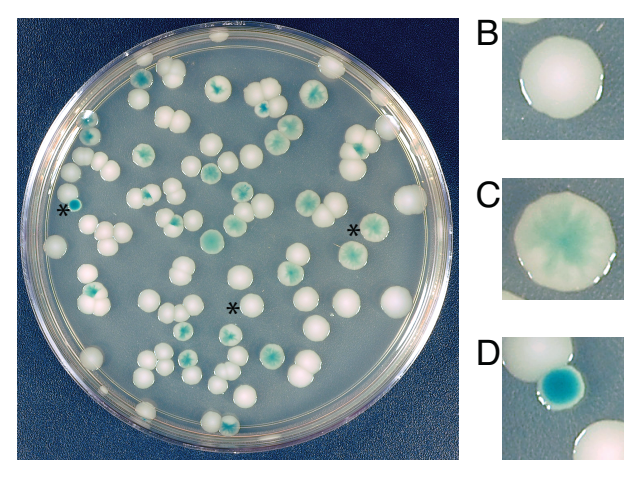

$\mathrm{E}$

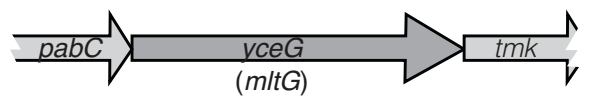

F

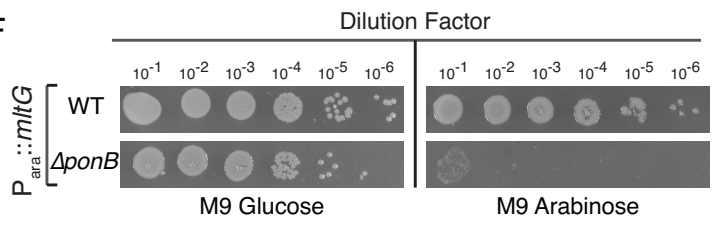

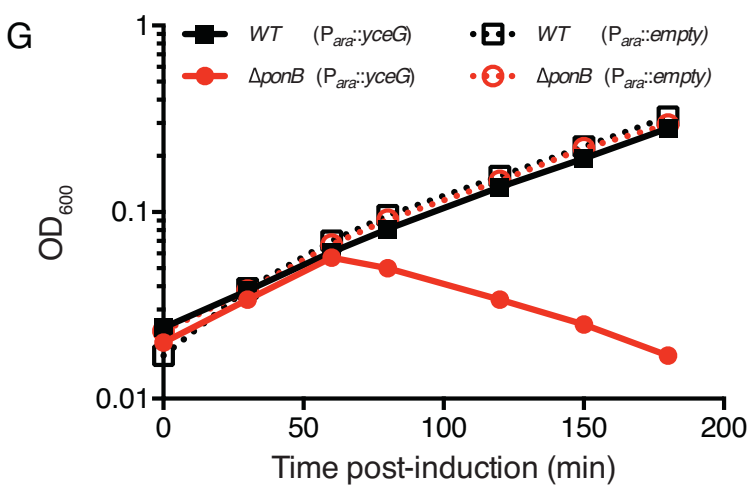
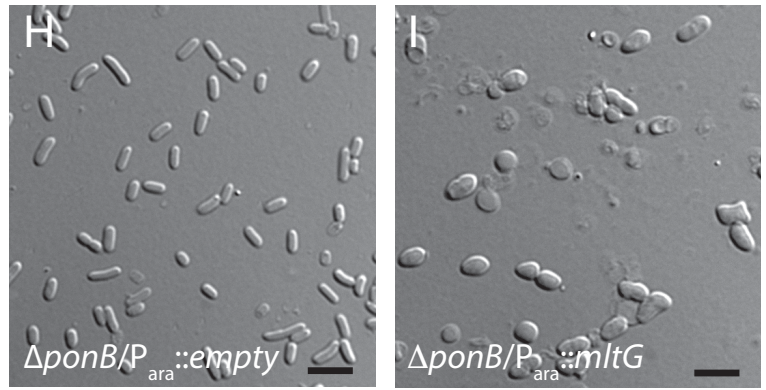

Figure 2.2. MltG overproduction is lethal in cells defective for PBP1b.

(A-D) Cells of TU122/pDY1 were transformed with the multicopy E. coli library and plated on LB agar supplemented with IPTG and Xgal. A typical screening plate is shown in panel A, and close-up images highlighting the different colony phenotypes are shown in (B-D). See text for details.

(E) Diagram of the genomic locus resulting in a multicopy lethal phenotype in $\triangle p o n B$ cells.

(F) Cells of MG1655 or its $\triangle p o n B$ derivative containing plasmid pRY53 [ $\left.\mathrm{P}_{\text {ara: }}: m l t G\right]$ were grown overnight in M9 minimal maltose medium supplemented with chloramphenicol. Cells in the resulting cultures were harvested, washed twice in an equal volume of M9 salts, and resuspended in $\mathrm{M} 9$ salts at an $\mathrm{OD}_{600}$ of 1.0. The resulting cell suspensions were subjected to serial dilution, and $5 \mu \mathrm{L}$ of each dilution were spotted onto M9 agar plates containing $0.2 \%$ glucose or arabinose as indicated. Plates were incubated at $30^{\circ} \mathrm{C}$ for 2 days prior to imaging.

(G) Overnight cultures of the strains in (F) as well as the same strains carrying an empty vector control were diluted 1:100 in M9 maltose medium with chloramphenicol and grown to mid-log with shaking at $37^{\circ} \mathrm{C}$. The cells were harvested, washed as above, and resuspended to an $\mathrm{OD}_{600}$ of 0.02 in $\mathrm{M} 9$ arabinose medium. Growth at $37^{\circ} \mathrm{C}$ was then monitored by following culture optical density $\left(\mathrm{OD}_{600}\right)$. 
Figure 2.2. (Continued). (H-I) $\triangle p o n B$ cells carrying either an empty vector pCM6 [ $\mathrm{P}_{\text {ara }}:$ empty] or plasmid pRY53 [ $\left.\mathrm{P}_{\text {ara }}:: m l t G\right]$ were grown on LB arabinose plates, and then scraped and resuspended in M9 salts for imaging on agarose pads using DIC optics. Bars equal 4 microns.

\section{MltG degrades PG}

MltG belongs to the YceG family of proteins in the Pfam database, which do not share sequence homology with proteins of known function, and are often misannotated as aminodeoxychorismate lyases ${ }^{34}$. The structure of $E$. coli $\mathrm{MltG}$ was solved several years ago as part of the structural genomics effort (PDB: 2r1f). It has an extended N-terminus topped by a globular head domain with an observable cleft where residues with the highest level of conservation are found (Figure 2.3A). To gain more insight into the potential function of MltG, we searched the Dali protein structure database ${ }^{35}$ for structural homologs. MltG was found to share structural features with the catalytic domain of SleB from Bacillus species (PDB: 4fet and $4 \mathrm{f} 55)^{36,37}$. SleB is a lytic transglycosylase important for degrading the PG cortex of Bacillus spores during germination ${ }^{38,39,40}$. Although SleB has a unique active site topology compared to other LTs, its catalytic domain displays a similar overall fold and shares similar catalytic residues with other LTs ${ }^{36,37}$. The SleB active site is located in a binding cleft at its C-terminus, within which lies the catalytic glutamate (E151) essential for SleB activity ${ }^{37}$. As previously reported by Jing and co-workers ${ }^{36}$, alignment of MltG with SleB showed a striking structural similarity in the catalytic cleft, including the placement of the likely catalytic glutamate residue (E218 in MltG) (Figure 2.3B). Thus, the available structural data suggested that MltG is likely to be a PG-processing enzyme with LT activity. 

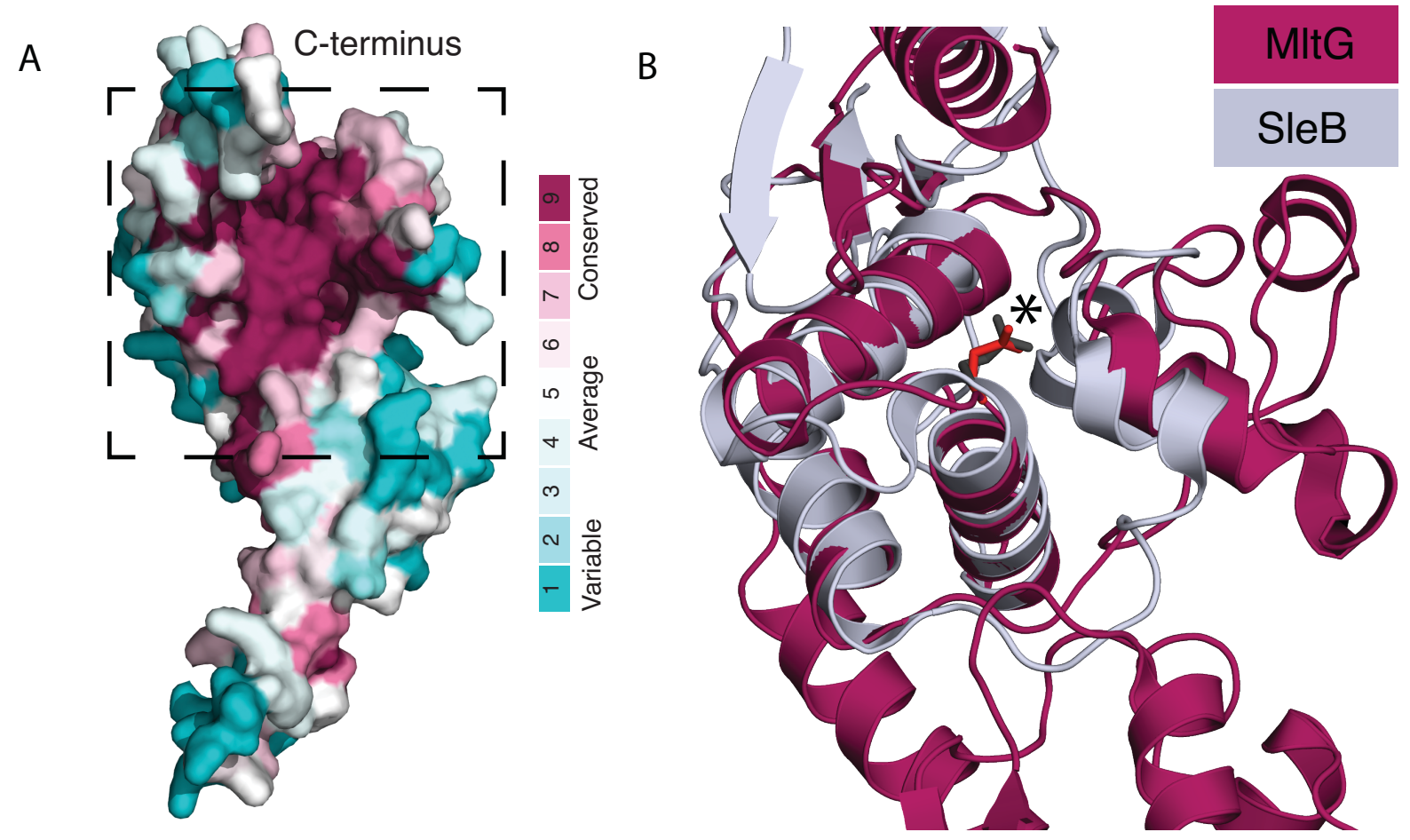

N-terminus

Figure 2.3. MltG is structurally similar to SleB.

(A) Shown is a space-filling model of the MltG structure (PDB 2r1f) colored to indicate amino acid conservation using ConSurf ${ }^{41}$. Note the high conservation of residues in the cleft-forming region.

(B) Ribbon diagrams showing the structural alignment of the proposed catalytic region of MltG (boxed in A) (burgundy) with the catalytic domain of the SleB lytic transglycosylase (grey) ${ }^{37,36}$. The catalytic glutamate is shown in stick form and highlighted with an asterisk. This structural homology was also reported previously by Jing and co-workers ${ }^{36}$. 
To test whether MltG degrades PG, a soluble form of the protein (sMltG) lacking its hydrophobic N-terminal domain was purified along with a control variant of sMltG with an E218Q substitution predicted to disrupt its catalytic activity. PG degradation was assessed using a dye-release assay for PG cleavage ${ }^{42}$. Purified E. coli PG sacculi were covalently labelled with the dye remazol brilliant blue (RBB) and incubated with the sMltG proteins $(4 \mu \mathrm{M})$ in addition to buffer and lysozyme controls. The reactions were terminated at different time points by heat inactivation $\left(95^{\circ} \mathrm{C}\right.$ for $\left.5 \mathrm{~min}\right)$, and any remaining intact $\mathrm{PG}$ was pelleted by centrifugation. The amount of dye released into the supernatant was then measured as a proxy for PG cleavage by determining the absorbance at $595 \mathrm{~nm}$. As expected, very little dye was released in the buffer control reaction at all incubation times, and lysozyme promoted a robust level of dye release after only 5 minutes of incubation with the RBB-labeled PG (Figure 2.4). Significant dye release by sMltG(WT) was observed after 18 hours of incubation (Figure 2.4). This weak PG degradation activity was unlikely to be due to a contaminating PG hydrolase because similarly purified sMltG(E218Q) was equivalent to the buffer control and did not display detectible dyerelease activity (Figure 2.4). Based on these results, we conclude that MltG is capable of cleaving PG and that the E218 residue is required for catalysis. 


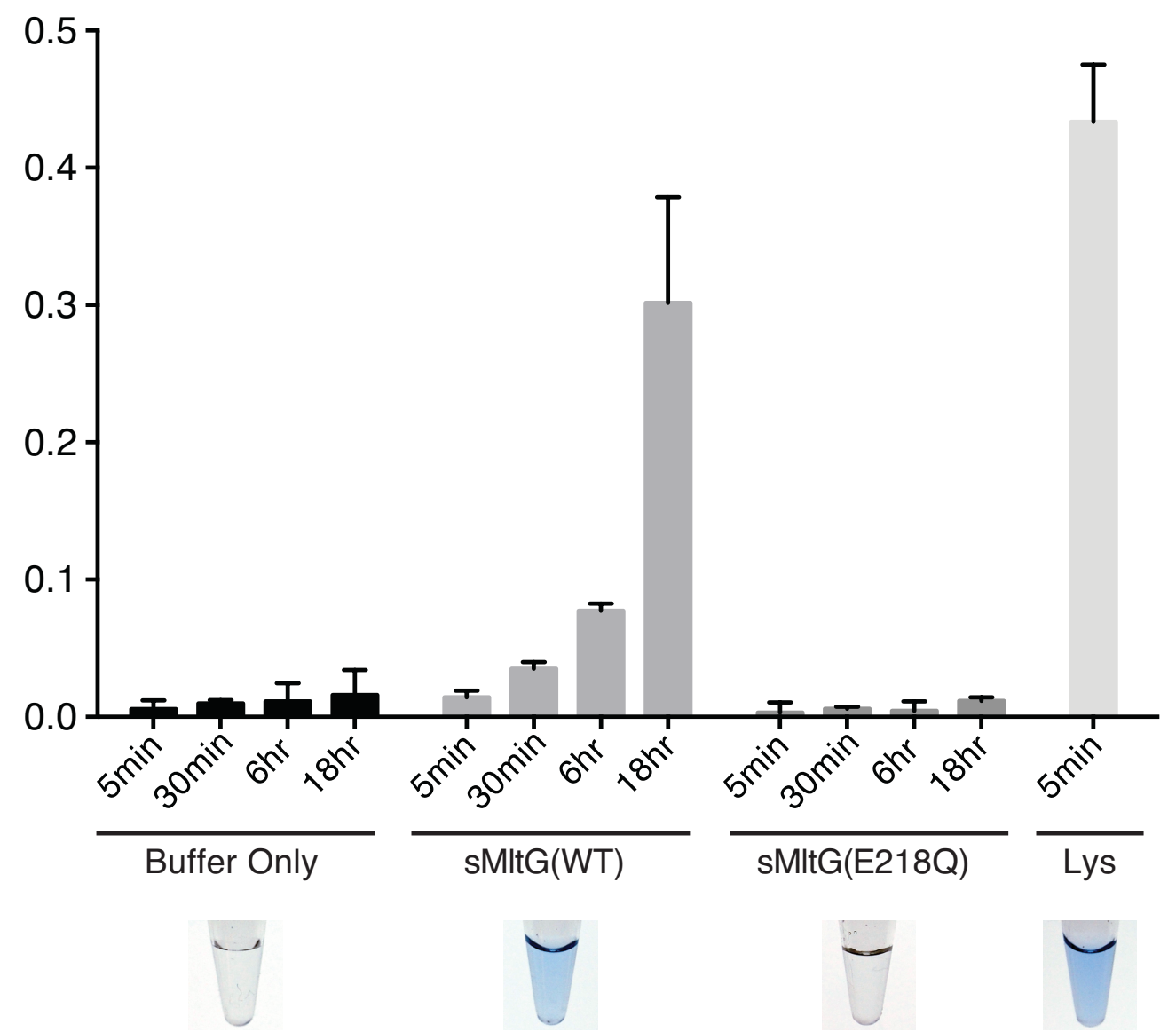

Figure 2.4. MltG degrades cell wall in vitro.

Dye-labeled PG sacculi were incubated with the indicated proteins $(4 \mu \mathrm{M})$ or buffer alone for the indicated times. Reactions were terminated, undigested PG was pelleted by centrifugation, and the absorbance of the supernatant at $595 \mathrm{~nm}$ was measured. Shown are the results from reactions performed in triplicate, with the error bars representing the $95 \%$ confidence interval of the measurements. 


\section{MltG is a lytic transglycosylase}

The structural relatedness of MltG to SleB suggested that it functions as an LT enzyme that cleaves glycan strands of PG with the concomitant formation of anh MurNAc-containing products (Figure 2.1). To determine the enzymatic specificity of sMltG, we used high performance liquid chromatography coupled with high resolution mass spectrometry (LC/MS) to analyze the digestion products of unlabeled E. coli $\mathrm{PG}$ treated with sMltG. The enzyme generated three main products, each with the same mass to charge ratio $(\mathrm{m} / \mathrm{z})$ of 922.389 , but increasing retention times and charge states of $+1,+2$, and +3 (Figure 2.5A and Figure 2.5C). An $m / z$ of 922.389 with a charge state of +1 is consistent with a GlcNAc-anhMurNActetrapeptide product formed via an LT cleavage reaction. Importantly, the same species was produced by the confirmed LT enzyme Slt ${ }^{43,44}$ (Figure 2.5B and Figure 2.5C). The additional products unique to the $\mathrm{sMltG}$ reaction with charged states of +2 and +3 are consistent with two possible anh MurNAc-containing products: (i) linear tetra- and hexasaccharide oligomers terminating with an ${ }^{\text {anh }}$ MurNAc end, or (ii) GlcNAc- ${ }^{\text {anh } M u r N A c-t e t r a p e p t i d e ~ f r a g m e n t s ~}$ engaged in a peptide crosslink with one or two normal (non-anhydro containing) GlcNAcMurNAc-tetrapeptide moieties.

To distinguish between these possibilities, sMltG reaction products were compared by LC/MS with and without prior reduction by sodium borohydride (Figure 2.6). Linear oligomers capped by ${ }^{\text {anh } M u r N A c ~ e n d s ~ a r e ~ n o n r e d u c i n g, ~ a n d ~ t h u s ~ s h o u l d ~ b e ~ u n a f f e c t e d ~ b y ~ s o d i u m ~}$ borohydride treatment. However, products terminating with normal MurNAc, as in the possible crosslinked products, are subject to reduction that is detectable as a small shift in the LC/MS profile and change in $\mathrm{m} / \mathrm{z}$ value. The sMltG products were unaffected by sodium borohydride treatment, indicating that they are likely to be linear oligomers capped by ${ }^{\text {anh MurNAc ends }}$ 
(Figure 2.6). Such products indicate that MltG is likely to be an endo-LT that cuts within glycan strands rather than an exo-enzyme like most other characterized LTs that process the glycan chain ends and only liberate monomeric or crosslinked GlcNAc-anhMurNAc-tetrapeptide products. We therefore conclude that MltG is an endolytic LT enzyme that cleaves within glycan strands. Furthermore, MltG did not appear to release cross-linked products from purified PG, suggesting that it favors uncrosslinked strands as a substrate.
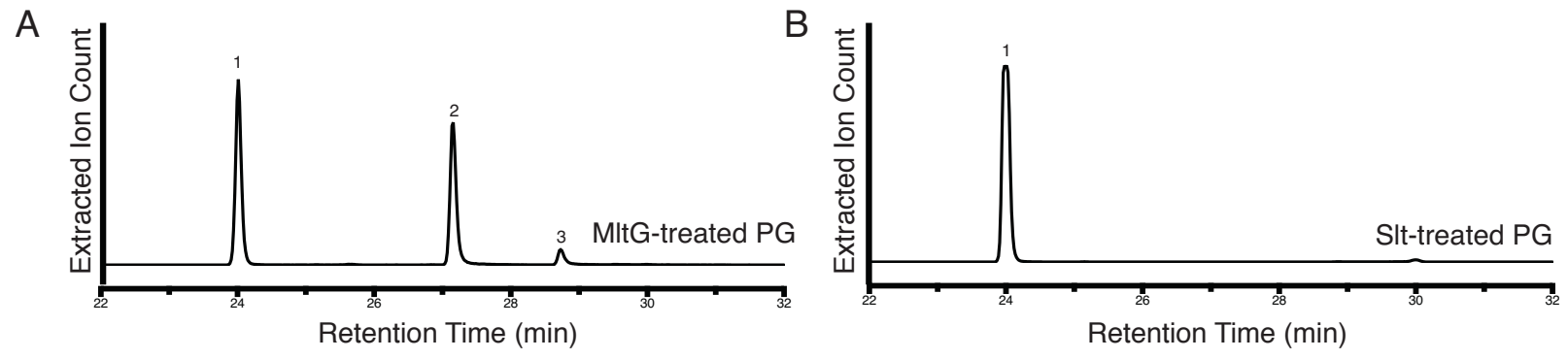

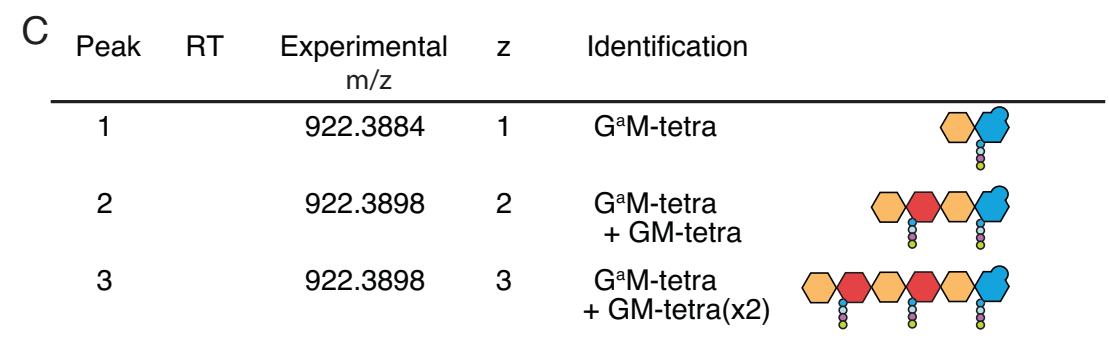

Figure 2.5. MltG is a lytic transglycosylase with endoglycosidase activity.

(A-B) Extracted-ion chromatograms resulting from the digestion of purified, unlabeled PG with MltG (A) or Slt (B). Traces are for ions with an $\mathrm{m} / \mathrm{z}$ of 922.38 corresponding to LT degradation products.

(C) Shows the identity of the peaks in (A-B) along with relevant mass data, including the experimental mass to charge ratio, charge (z), peak identity, and cartoon structure of product molecules. 

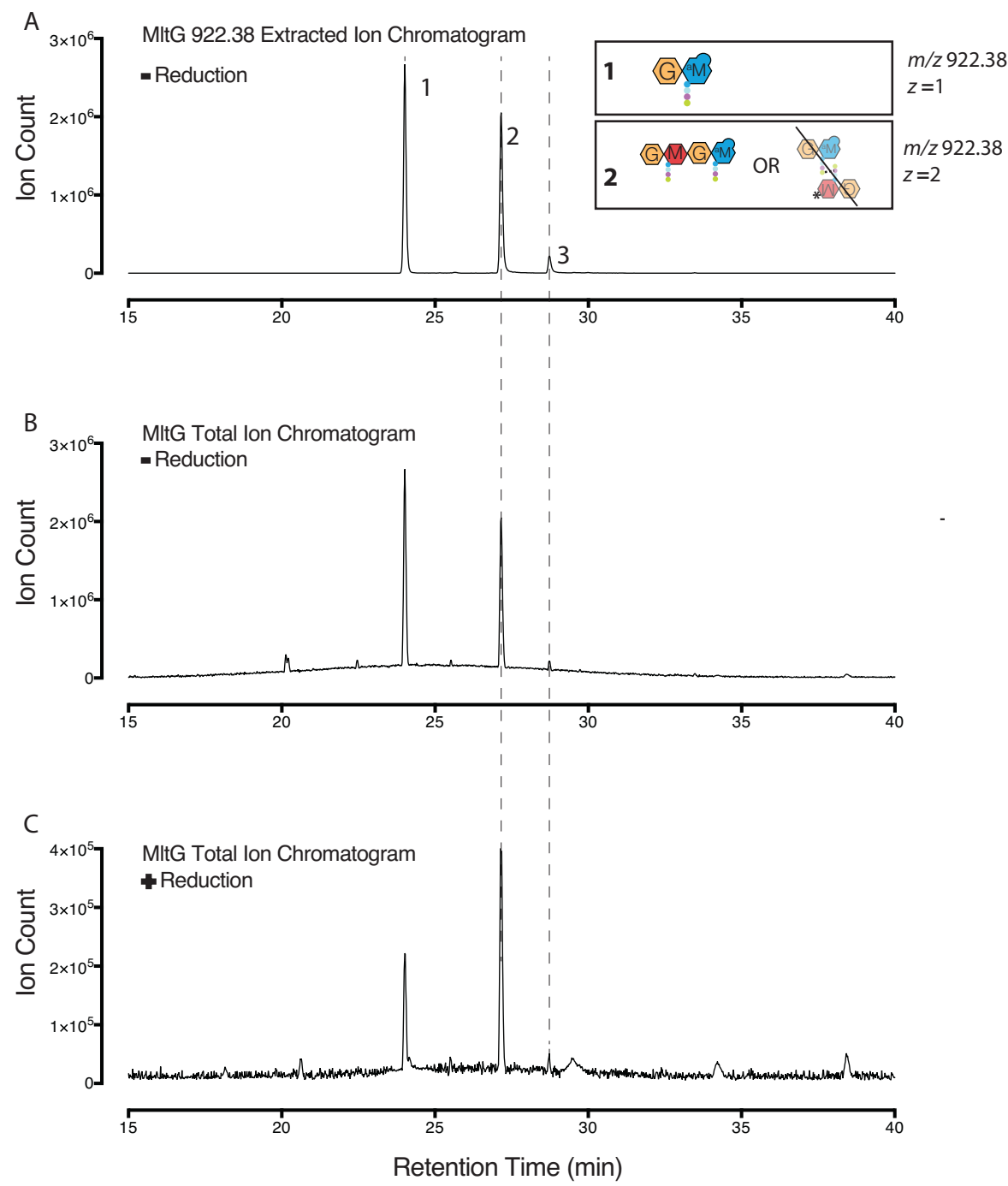

\section{Figure 2.6. MltG generates muropeptides with non-reducing termini.}

Extracted-ion chromatogram for $922.38 \mathrm{~m} / z$ (see Figure 2.5A) and Total-ion chromatograms (TIC) resulting from the digestion of purified, unlabeled PG with MltG. (B) and (C) chromatograms compare the total products observed with and without sodium borohydride reduction, which reduces the MurNAc sugar in a GlcNAc-MurNAc disaccharide to muramitol. If there is a free reducing end, there will be a $2 \mathrm{Da}$ difference after reduction and a slight shift in retention time. If there is no reducing end, the products will not shift in mass and there will be no observed shift in retention time. Products corresponding to $922.389 \mathrm{~m} / \mathrm{z}$ (A) align with the primary peaks in the TIC. 
Figure 2.6 (Continued). (B). These peaks do not shift in mass or retention time with reduction indicating that the three primary products generated by MltG have non-reducing termini, a property consistent with muropeptides that lack a free $\mathrm{N}$-acetylmuramic acid and are capped by 1,6-anhydro- $N$-acetylmuramic acid. As shown in the inset in (A), two possible products could result in the same $m / z$ ratio of 922.38 with $z=2$, one oligomeric and one cross-linked. However, the cross-linked product has a reducing end (indicated by the *), whereas the oligomeric product with the anhydro cap does not. Therefore, only the oligomeric product would resist reduction as observed with the larger products generated by MltG. A similar pattern and conclusion has been observed for the products released by MltE, a previously characterized endo-acting lytic transglycosylase ${ }^{45}$.

\section{MltG is part of a widely conserved LT enzyme family}

According to the Pfam database, about $70 \%$ of bacterial genomes $(2167$ species out of 3060 sequenced bacterial genomes), including many species of firmicutes, proteobacteria, and actinobacteria, produce proteins that contain $\mathrm{MltG}(\mathrm{YceG})$-like domains ${ }^{34}$. To better assess the conservation of MltG-like proteins relative to previously characterized LTs, the presence or absence of all LT families identified to date was assessed across a diverse set of 150 representative bacterial species ${ }^{46}$ (Figure 2.7). MltG-like proteins showed a high degree of conservation, being present in 113/150 (75\%) bacterial genomes analyzed (Figure 2.8). The only other family with a similar degree of conservation was the Slt superfamily, including Slt, MltC, MltD, MltE, and MltF in E. coli (Figure 2.8). With the exception of the RlpA family, all other LT families were present in less than half of the species analyzed. MltG family members as well as the less well conserved SleB proteins are unique in that they are more evenly distributed between gram-negative and gram-positive bacterial genomes relative to the other families, which are largely skewed towards the gram-negatives (Figure 2.8). The conservation profile of MltGlike proteins suggests that these LTs likely play a fundamental role in cell wall processing in a large fraction of bacteria (see Discussion). 


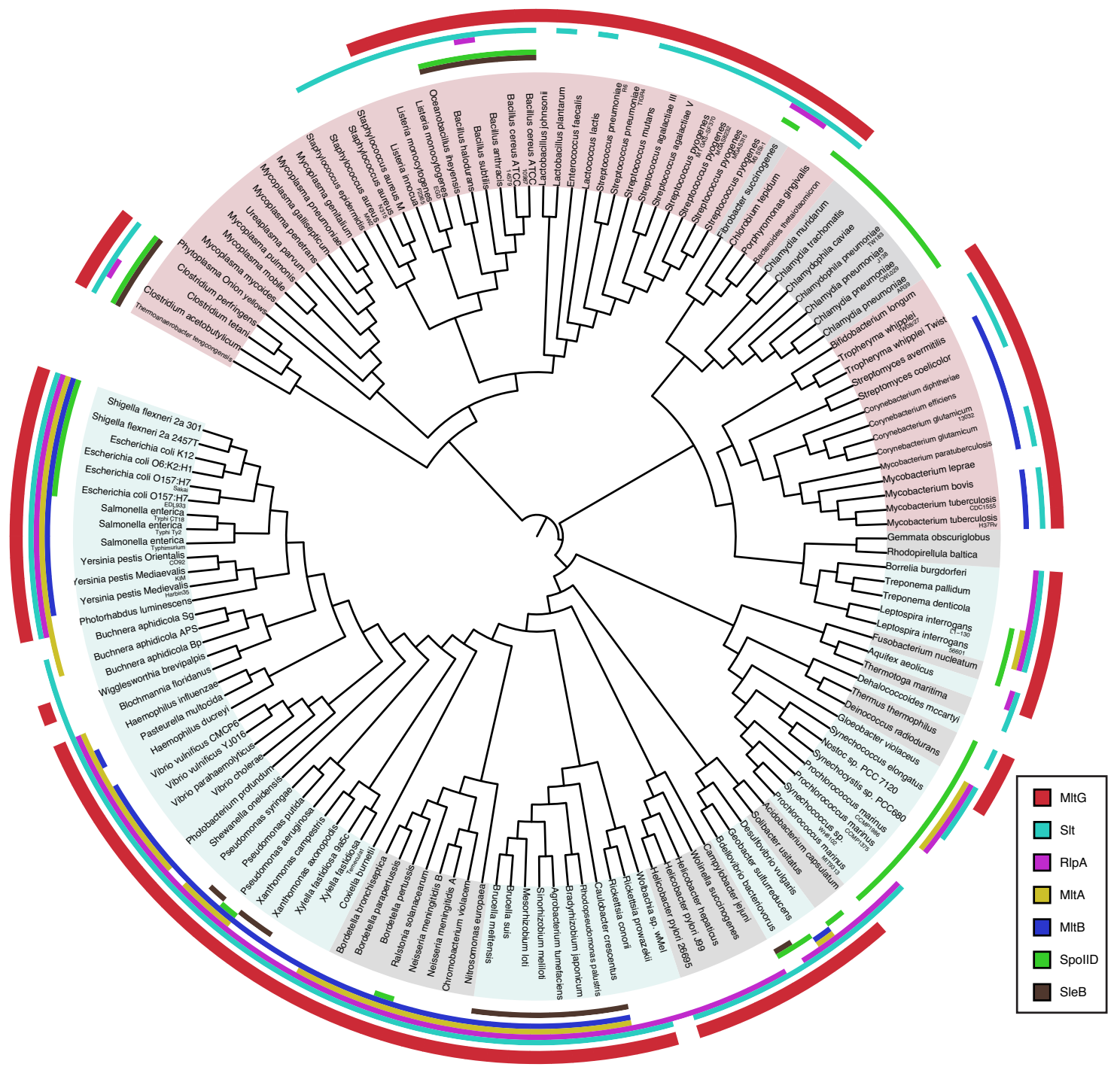

Figure 2.7. MltG is widely conserved in the bacterial domain.

Bacterial phylogenetic tree constructed using iTOL ${ }^{47}$ and a diversity set of 150 strains. The presence of MltG or other LT family members in a given species is indicated by the colored regions at the outer edge of the tree. A color-coded legend is given in the lower right portions of the panel. 
A

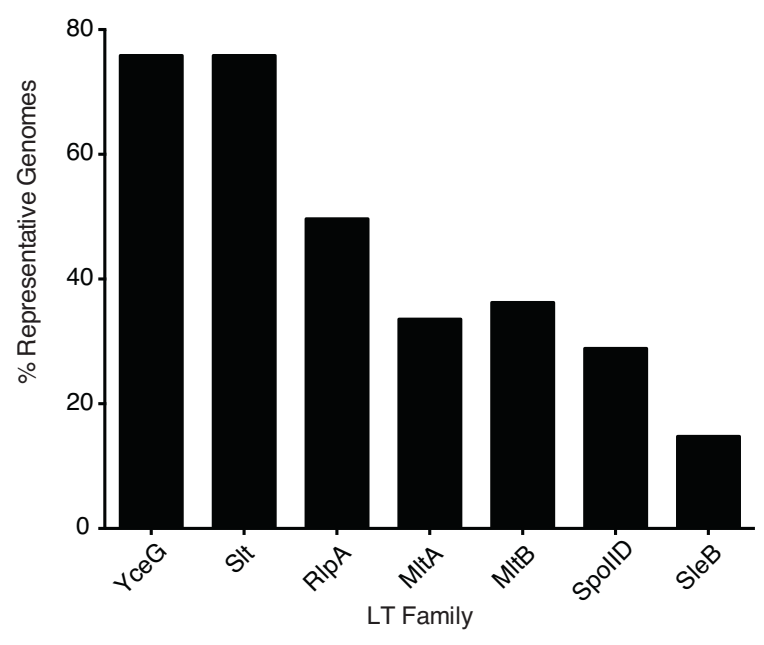

B

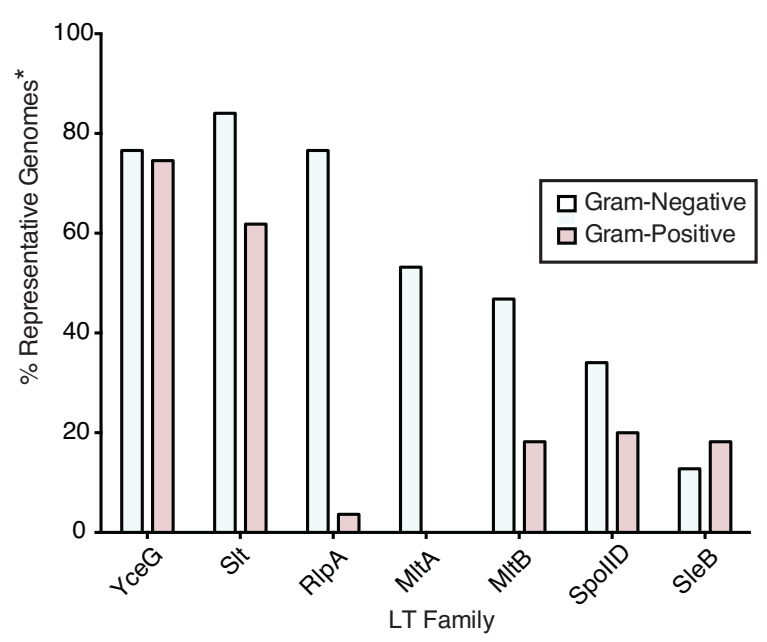

\begin{tabular}{|c|c|c|}
\hline LT Family & $\begin{array}{l}\text { Pfam } \\
\text { Identifier }\end{array}$ & Pfam Name \\
\hline SIt & PF01464 & Transglycosylase SLT domain \\
\hline MltG & PF02618 & YceG-Like domain \\
\hline MltA & $\begin{array}{l}\text { PF03562 } \\
\text { (w/ PF06725) }\end{array}$ & MltA specific insert domain \\
\hline MltB & PF13406 & SLT_2: Transglycosylase SLT domain \\
\hline RIpA & PF03330 & DPBB_1: Rare lipoprotein A (RlpA)-like double-psi beta-barrel \\
\hline SleB & PF07486 & Hydrolase_2: Cell Wall Hydrolase \\
\hline SpollD & PF08486 & SpolID: Stage II sporulation protein \\
\hline
\end{tabular}

Figure 2.8. Conservation profiles of MltG and other LTs.

(A) Graph showing the number of genomes in which each LT family is found out of the total 150 used in the diversity set.

(B) Graph showing the percentage of gram-negative vs gram-positive organisms in the dataset containing each LT family. Gram-negative and gram-positive organisms were assessed as independent groups given their unequal representation in the original genome list. Percentages in (B) were calculated using the equations \% gram-negative $=(\#$ of gram-negative organisms with gene $\mathrm{X} / \#$ of gram-negatives $)^{*} 100$ and \% gram-positive $=(\#$ of gram-positive organisms with gene $\mathrm{X} /$ \# of gram-positives)*100.

(C) Table outlining the primary Pfams found in each family of LTs. These Pfams were used to identify potential members of each LT family analyzed in Figure 2.7. 


\section{MltG localizes to the inner membrane of $E$. coli.}

All of the previously characterized LTs in E. coli as well as RlpA are reported to either be soluble periplasmic proteins (Slt) or outer membrane-anchored proteins (MltA-F and RlpA) ${ }^{14,15}$. Depending on the algorithm used, TMHMM $^{48}$ or Phobius ${ }^{49}$, the N-terminal 20-25 amino acids of MltG are predicted to either encode a transmembrane helix or a signal sequence for export, respectively. A cytological assay ${ }^{50}$ was therefore used to experimentally determine whether MltG resides in the inner membrane or periplasm. Cells expressing an MltG-mCherry fusion were hyperosmotically shocked to induce plasmolysis and visualized using fluorescence microscopy. Upon plasmolysis, the cytoplasm shrinks and the inner membrane pulls away from the rest of the envelope. The pattern of MltG-mCherry fluorescence followed the retracted inner membrane and closely resembled that of a control mCherry fusion to a lipoprotein signal sequence from Pal modified for inner membrane retention (Figure 2.9A-D). In contrast, mCherry fused to the native Pal signal sequence for outer membrane (OM) targeting displayed a smooth peripheral fluorescence signal in the plasmolyzed cells consistent with an OM localization (Figure 2.9A-D). The fluorescent signal of the periplasmic Slt-mCherry control, on the other hand, accumulated in the enlarged periplasmic compartments of the plasmolysis bays, which appear phase-light in the bright field images (Figure 2.9A-D). Based on the cytological results, we conclude that MltG is localized to the inner membrane. 

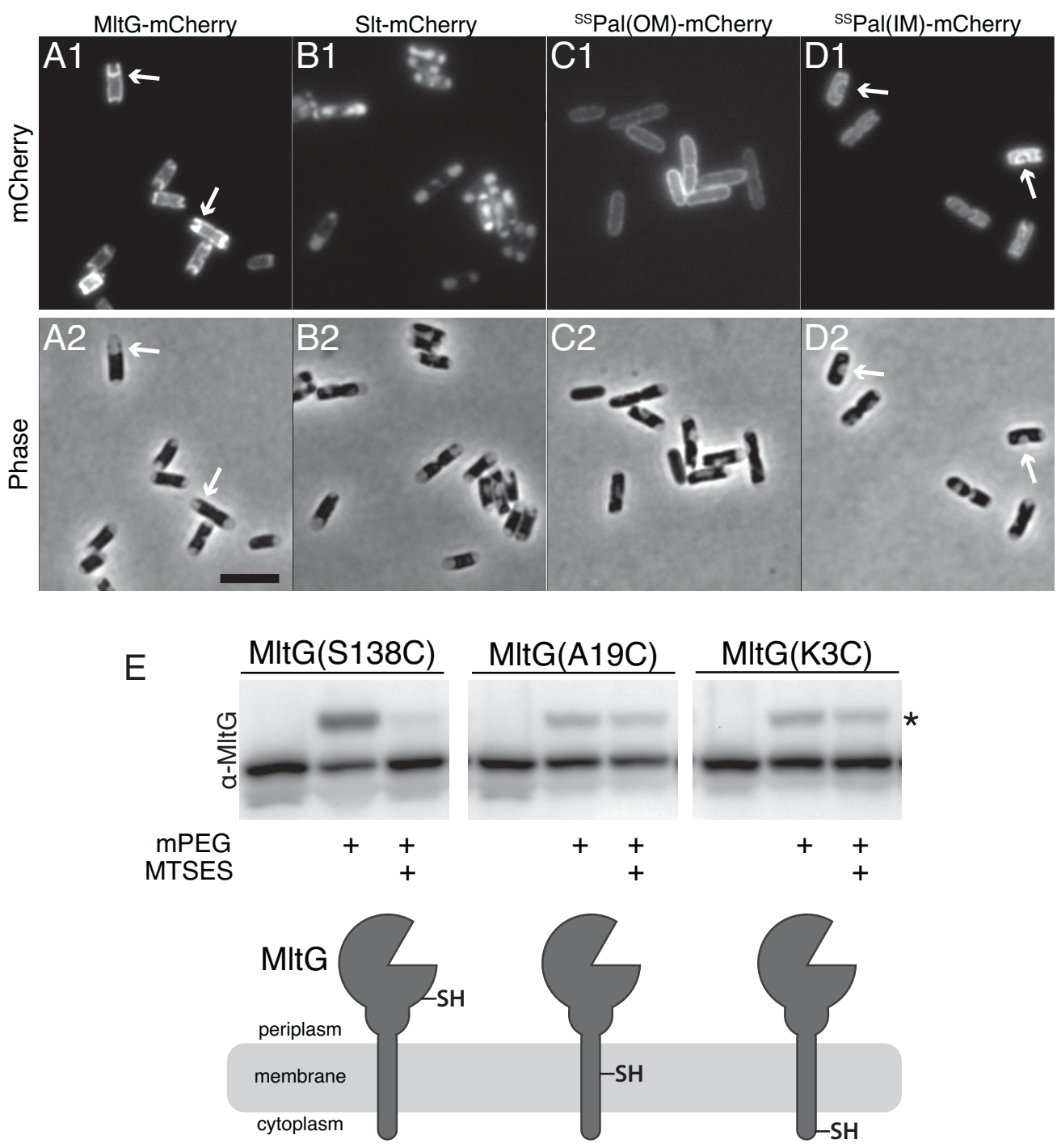

\section{Figure 2.9. MltG is an inner membrane protein.}

(A-D) Cytological assay for determining the localization of MltG in the cell envelope. Cells expressing mCherry fusions to MltG (A), Slt (B), the wildtype signal-sequence of Pal for outer membrane targeting $\left[{ }^{\mathrm{ss}} \mathrm{Pal}(\mathrm{OM})\right](\mathbf{C})$, or a modified version of ss Pal retained in the inner membrane [ss Pal(IM)] (D) were grown to an $\mathrm{OD}_{600}$ of $0.3-0.4$, osmotically shocked by resuspension in plasmolysis buffer (30\% sucrose, $50 \mathrm{mM}$ HEPES (pH 7.4), $40 \mathrm{mM}$ sodium azide), and imaged by mCherry (panel 1) or phase contrast (panel 2) optics. Slt-mCherry serves as a periplasmic control. ${ }^{\mathrm{ss}} \mathrm{Pal}(\mathrm{OM})$-mCherry and ${ }^{\mathrm{ss}} \mathrm{Pal}(\mathrm{IM})-\mathrm{mCherry}$ serve as controls for outer and inner membrane localized proteins, respectively. Arrows highlight signals that track with the inner membrane in plasmolysis bays. Bar equals 4 microns. 
Figure 2.9 (Continued). (E) Cells expressing the indicated variants of MltG were harvested $1 \mathrm{hr}$ post induction with $100 \mu \mathrm{M}$ IPTG. The whole cells were left either untreated or treated with 10mM MTSES. After washing out excess MTSES, treated and untreated cells were lysed, their proteins were denatured, and the lysates were exposed to $5 \mathrm{mM}$ PEG-malemide (mPEG) (2kDa). Following TCA precipitation, equivalent amounts of total protein were separated by SDS-PAGE and blotted to membranes. MltG variants were detected with affinity purified anti-MltG antibodies. A cartoon diagram of the MltG membrane topology suggested by the SCAM analysis is shown below the blot image with the location of the Cys residue in each variant indicated. Asterisk marks the bands of MltG shifted to a higher molecular weight following reaction with mPEG.

TMHMM predicts that residues 1-4 of MltG reside in the cytoplasm followed by a transmembrane helix (a.a. 5-24) and a large periplasmic domain with the LT active site (a.a. 25340). To experimentally validate this topology, the scanning cysteine accessibility method (SCAM) was employed ${ }^{51,52}$. Cells expressing MltG variants with single cysteine substitutions at positions 3 , 19, and 138 were treated with the (inner) membrane-impermeable sulfhydrylreactive reagent (2-sulfonatoethyl) methanethiosulfonate (MTSES). MTSES reacts with cysteines exposed to the periplasm or cell surface, but not those in the cytoplasm or buried in the membrane. MTSES accessibility was then determined by lysing the cells and treating with a second sulfhydryl-reactive reagent, a 2 kDa malemide-PEG derivative (mal-PEG). This compound reacts with cysteines that have not already been "protected" by MTSES, producing a $2 \mathrm{kDa}$ shift in molecular weight that can be detected on an immunoblot. A fraction of both MltG(K3C) and MltG(A19C) reacted with mal-PEG and were shifted in the immunoblot whether or not cells were treated with MTSES before lysis (Figure 2.9E). Conversely, MltG(S138C) reacted robustly with mal-PEG, but this reaction was almost completely blocked by MTSES pretreatment (Figure 2.9E). The SCAM results are therefore consistent with MltG being a single 
pass transmembrane protein with an $\mathrm{N}$-in/C-out topology. Thus, $\mathrm{MltG}$ is unique among the characterized LTs of E. coli and other gram-negative bacteria in that it is anchored in the inner membrane.

\section{MltG is required for normal glycan strand length distribution}

As for all other $E$. coli LTs, inactivation of $m l t G$ alone does not result in a significant growth or morphological phenotype (data not shown). However, given the unique location of MltG in the inner membrane along with its endoglycosidase activity, we suspected that the loss of MltG function might alter the chemical composition of the PG matrix, particularly its

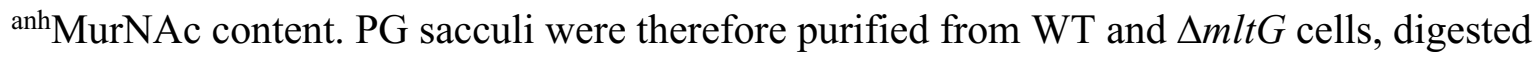
with the muramidase mutanolysin, and the resulting muropeptide fragments were analyzed by LC/MS. Although the muropeptide composition of the PG from the two strains was largely similar, significant differences in a subset of muropeptides were observed (Figure 2.10, Figure 2.11, and Table 2.1). The largest percentage change was observed for uncrosslinked muropeptides with a pentapeptide stem, rising 252\% in $\Delta m l t G$ cells (Figure 2.10A, Figure 2.11, and Table 2.1). However, due to the extremely low pentapeptide content of WT PG, this change is small in absolute terms and of unclear significance. A more informative change was observed for anhydromuropeptides, which decreased in total by $20 \%$ in $\Delta m l t G$ cells relative to WT, with a $16 \%$ reduction in monomeric anhdyromuropeptides, a $26 \%$ reduction in dimeric (crosslinked) muropeptides with a single ${ }^{\text {anh }}$ MurNAc, and 43\% reduction in trimeric (multiply crosslinked) anhydromuropeptides (Figure 2.10A, Figure 2.11, and Table 2.1). 
A

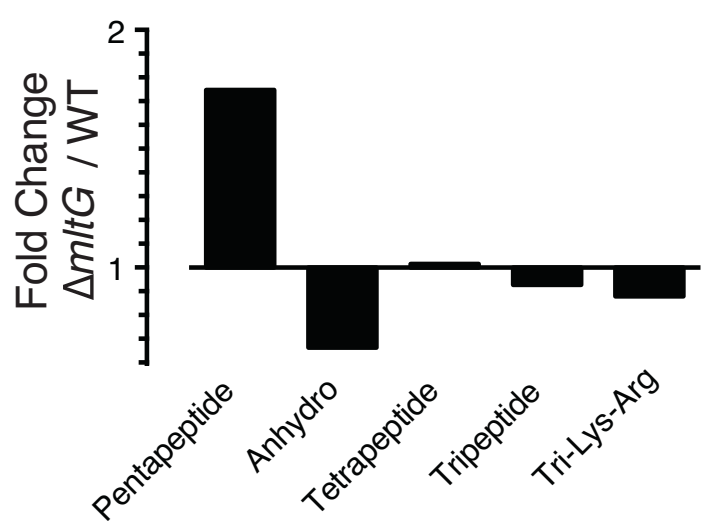

B

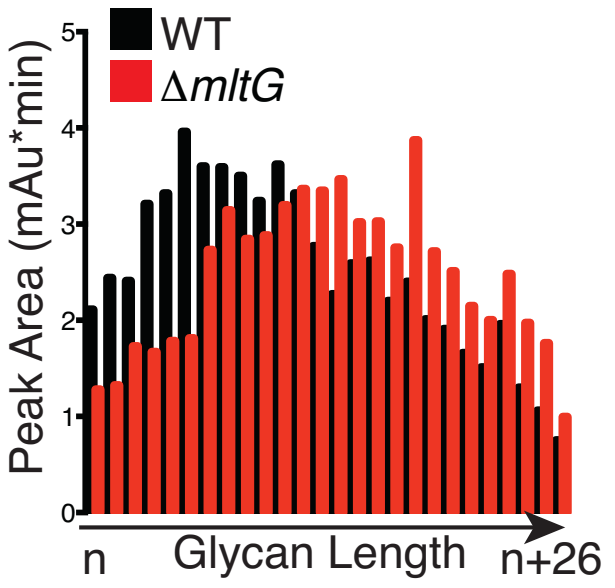

Figure 2.10. Alteration of muropeptide composition and glycan strand length in the PG of MItG defective cells.

(A) The abundance of each muropeptide species in WT and $\Delta m l t G$ PG preparations was determined. Shown are the ratios of selected groupings of muropeptide species in $\Delta m l t G$ versus WT PG. Pentapeptide, Tetrapeptide, and Tripeptide indicate all muropeptide species (crosslinked and uncrosslinked) containing the indicated type of peptide. Similarly, Anhydo indicates all anhMurNAc containing species, and Tri-Lys-Arg indicates species that were linked to the outer membrane lipoprotein Lpp. Tabulated relative abundances for the complete set of known muropeptide species for each strain are presented in Table 2.1.

(B) PG preparations from the indicated strains were treated with the amidase AmiD to remove the peptide stems. The resulting peptide-free glycan strands were then separated according to length and quantified using a previously described HPLC protocol ${ }^{53}$. Quantified peak areas for representative samples are shown. Chromatograms are presented in Figure 2.12. 


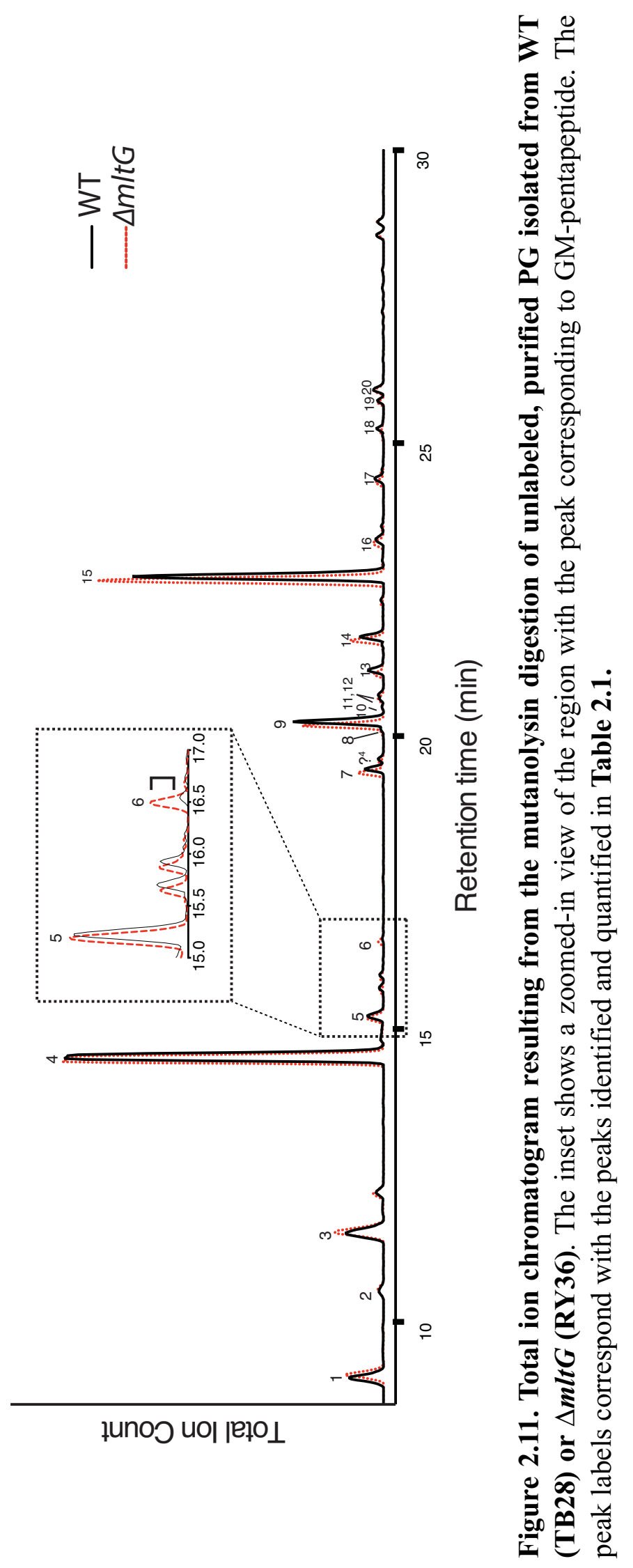


Table 2.1. Muropeptide analysis. Table listing the major muropeptide species identified by LC/MS following mutanolysin digest of WT (TB28) or $\Delta m l t G$ (RY36) sacculi. Peak numbers correspond to the labeled peaks shown in Figure 2.11. $\mathrm{GM}^{\mathrm{R}}$ indicates a GlcNAc-MurNAc disaccharide with the MurNAc sugar reduced to muramitol for the LC/MS analysis. Peptide length is given following the dash (-) and crosslinks are indicated with a slash (/). Peaks marked with a $\left(^{*}\right)$ could correspond with either the shown species (A / B) or the reciprocal dimer (B / A). The percent of total of muropeptides was calculated from the extracted ion counts for each muropeptide species after normalization for total muropeptide levels in each sample. Percent change was calculated by the following formula: [\% Area $(\Delta m l t G)$ - \% Area (WT) ] / \% Area $(\mathrm{WT}) * 100$. All values were determined through analysis of three biological replicates from sacculi isolated from cultures grown from independent colonies. Other minor muropeptides species not shown in the table were also identified and included in the summary calculations shown in Figure 2.10 and Table 2.2. 


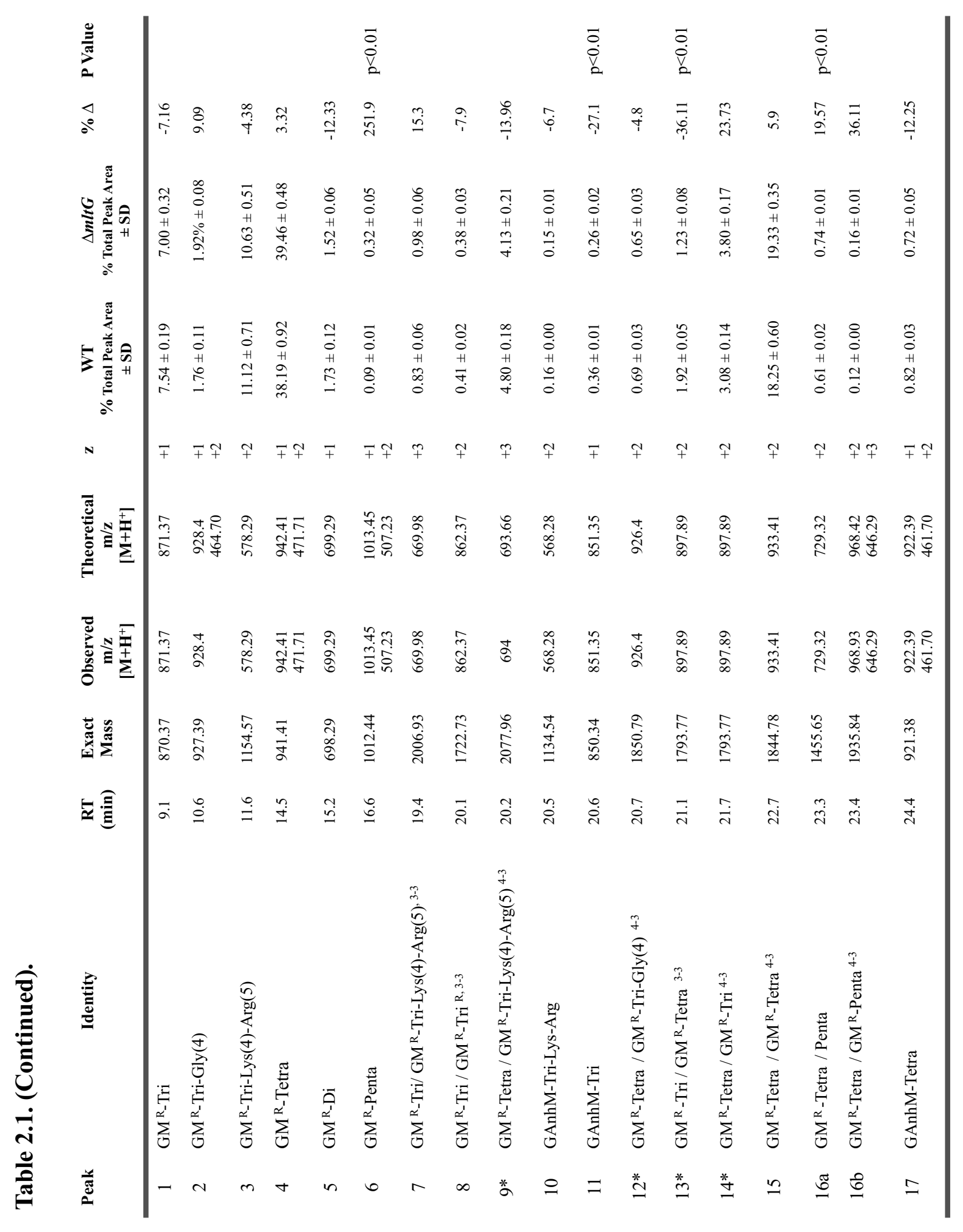


Table 2.2. Summary of the muropeptide composition of WT and $\Delta m l t G$ sacculi.

Table summarizing the major categories of muropeptides, organized by (A) glycan composition and cross-linkage and (B) stem peptide composition and cross-linkage. Values represent percentage of total muropeptides calculated from the LCMS muropeptide analysis described in Figure 2.10 and Table2.1/Figure 2.11.

A

\begin{tabular}{|c|c|c|c|}
\hline & WT & $\Delta m l t G$ & $\% \Delta$ \\
\hline Un-crosslinked & $60.01 \%$ & $60.05 \%$ & $0.07 \%$ \\
\hline GM & $58.68 \%$ & $58.93 \%$ & $0.43 \%$ \\
\hline GAnhM & $1.33 \%$ & $1.12 \%$ & $-15.79 \%$ \\
\hline Dimers & $31.95 \%$ & $32.17 \%$ & $0.69 \%$ \\
\hline GM/GM & $30.02 \%$ & $30.75 \%$ & $2.43 \%$ \\
\hline GM/GAnhM & $1.93 \%$ & $1.42 \%$ & $-26.42 \%$ \\
\hline Trimers & $1.47 \%$ & $1.23 \%$ & $-16.33 \%$ \\
\hline GM/GM/GM & $1.26 \%$ & $1.12 \%$ & $-11.11 \%$ \\
\hline GAnhM/GM/GM & $0.21 \%$ & $0.12 \%$ & $-42.86 \%$ \\
\hline All GM & $91.07 \%$ & $91.59 \%$ & $0.57 \%$ \\
\hline All GAnhM & $2.37 \%$ & $1.87 \%$ & $-21.1 \%$ \\
\hline
\end{tabular}

B

\begin{tabular}{lccc} 
& WT & $\Delta \boldsymbol{m l t G}$ & $\mathbf{\%} \Delta$ \\
\hline Tri & $\mathbf{1 1 . 2 6 \%}$ & $\mathbf{1 0 . 6 7 \%}$ & $\mathbf{- 5 . 2 4 \%}$ \\
Monomer & $7.9 \%$ & $7.3 \%$ & $-7.59 \%$ \\
Dimer & $3.3 \%$ & $3.4 \%$ & $3.03 \%$ \\
Trimer & $0.03 \%$ & $0.04 \%$ & $33.33 \%$
\end{tabular}

\begin{tabular}{lccc} 
Tri-Lys-Arg & $\mathbf{2 3 . 4 9 \%}$ & $\mathbf{2 1 . 6 \%}$ & $\mathbf{- 8 . 0 5 \%}$ \\
Monomer & $11.28 \%$ & $10.78 \%$ & $-4.43 \%$ \\
Dimer & $12.12 \%$ & $10.76 \%$ & $-11.22 \%$ \\
Trimer & $0.1 \%$ & $0.06 \%$ & $-37.76 \%$ \\
& & & \\
Tetra & $\mathbf{6 5 . 3 7 \%}$ & $\mathbf{6 6 . 0 6} \%$ & $\mathbf{1 . 0 6 \%}$ \\
Monomer & $39.01 \%$ & $39.46 \%$ & $1.16 \%$ \\
Dimers & $25.02 \%$ & $25.47 \%$ & $1.77 \%$ \\
Trimers & $1.34 \%$ & $1.13 \%$ & $-15.38 \%$ \\
& & & \\
Penta & $\mathbf{0 . 4 6 \%}$ & $\mathbf{0 . 7 7 \%}$ & $\mathbf{6 7 . 3 9 \%}$ \\
Monomer & $0.09 \%$ & $0.32 \%$ & $255.56 \%$ \\
Dimers & $0.37 \%$ & $0.45 \%$ & $21.62 \%$ \\
\hline
\end{tabular}


Given that anhydromuropeptides cap the strands, the reduction in anhydromuropeptide content of PG from $\Delta m l t G$ cells suggests an overall increase in glycan strand length. Consistent with this interpretation, when glycan strand length was directly measured in WT and $\Delta m l t G$ cell walls, a reproducible shift towards longer glycan strands was observed in strains lacking MltG (Figure

\subsection{B and Figure 2.12).}

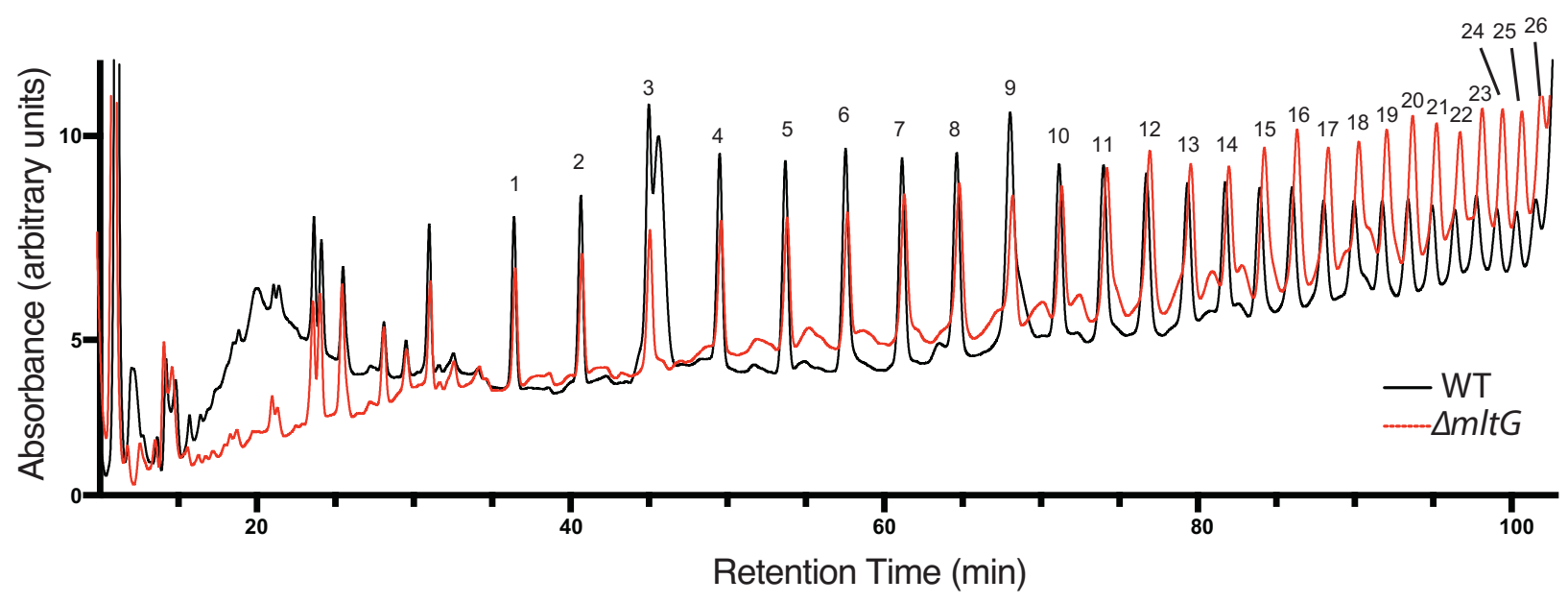

Figure 2.12. Overlaid chromatograms showing the peaks associated with peptide-free glycan strands released from AmiD-treated sacculi isolated from WT (TB28, black) and $\Delta m l t G$ (RY36, red). The resulting peptide-free glycan strands were then separated according to length using a previously described HPLC protocol ${ }^{53}$. Peaks increase in length with increasing elution times. However, here, the precise number of disaccharides per peak was not determined as the HPLC conditions were incompatible with mass spectrometry. The integrated area under the peaks numbered 1-26 correspond with the graph shown in Figure 2.10B. 


\section{MltG interacts with PBP1b}

The localization of MltG in the inner membrane places it in a position to interact with the similarly localized PG synthases as a potential means to coordinate PG synthesis with MltGcatalyzed processing of glycan strands. To probe for potential interactions between MltG and the aPBP synthases, we performed bacterial two hybrid (BACTH) analysis based on the splitadenylate cyclase fragments T18 and T2 ${ }^{54}$. In this assay, if two proteins interact, the T18 and T25 fragments are brought together to form a functional adenylate cyclase that can correct an $E$. coli cyaA defect by promoting cyclic-AMP (cAMP) production. This complementation is detectable via $l a c Z$ induction and the formation of blue color when cells are grown on agar containing X-gal. Production of T18-MltG led to a positive interaction signal in combination with T25-PBP1b and T25-MltG, but not with T25-PBP1a or the T25-Zip control (Figure 2.13). Similar results were obtained with the T25-MltG fusion; it showed a positive signal with T18PBP1b, but not with T18-PBP1a or T18-Zip (Figure 2.13). Based on these results we conclude that MltG interacts, directly or indirectly, with the PG synthase PBP1b and also self-interacts. The self-interaction is consistent with the dimeric form observed in the MltG crystal structure

\begin{tabular}{|ll|}
\hline T25 $\frac{\mathrm{T} 18-Y_{c e G}}{}$ & T18 $\frac{\mathrm{T} 25-\mathrm{YceG}_{1}}{\square}$ \\
Empty & Pmpty \\
PBP1a & \\
PBP1b & \\
PceG & \\
\hline
\end{tabular}

\section{Figure 2.13. Bacterial two-hybrid interaction of MltG with PBP1b.}

BTH101 cells containing plasmids producing the indicated T25 and T18 fusions were grown to saturation in LB with $50 \mu \mathrm{g} / \mathrm{ml} \mathrm{amp}, 25 \mu \mathrm{g} / \mathrm{ml} \mathrm{kan}$, and $500 \mu \mathrm{M}$ IPTG and $5 \mu 1$ of each culture was spotted on LB agar containing $40 \mu \mathrm{g} / \mathrm{ml} \mathrm{Amp,} 25 \mu \mathrm{g} / \mathrm{ml} \mathrm{kan}, 500 \mu \mathrm{M}$ IPTG, and $40 \mu \mathrm{g} / \mathrm{ml}$ X-gal. 
(PDB: 2r1f). Whether MltG also interacts with PBP1a is not clear as a negative result in the BACTH assay is not interpretable without additional experimentation. Nevertheless, the BACTH results connect MltG with at least one PG synthase in the inner membrane.

\section{MltG processes nascent PG in vivo}

Our results are consistent with a model in which MltG interacts with PBP1b, and possibly other PG synthases, to process nascent PG strands. To investigate the ability of MltG to cleave nascent PG, we used an in vivo labeling method that detects the turnover of nascent material following beta-lactam treatment. Beta-lactams uncouple glycan chain synthesis from crosslinking by blocking the TP activity of their target PBPs. This uncoupling leads to the degradation of nascent uncrosslinked PG produced by the crippled synthetic machinery ${ }^{55,16}$. Following specific inhibition of the bPBPs PBP2 or PBP3 with the drugs mecillinam or cephalexin, respectively, we found that the periplasmic LT Slt was the main enzyme responsible for the destruction of uncrosslinked $\mathrm{PG}^{16}$. However, for the beta-lactam cefsulodin, which specifically targets the aPBPs PBP1a and PBP1b ${ }^{56}$, nascent PG degradation was only reduced by $50 \%$ upon Slt inactivation. This observation suggested that at least one other LT contributes to the cefsulodin-induced degradation of nascent, uncrosslinked PG. We suspected that this LT might be MltG, and therefore monitored cell wall synthesis and turnover in cells inactivated for Slt, MltG, or both enzymes with or without prior cefsulodin treatment.

PG synthesis and turnover can be monitored by labeling cells with [3H]-mesodiaminopimelic acid (mDAP), an amino acid unique to the peptide moiety of PG. Following $[3 \mathrm{H}]-\mathrm{mDAP}$ addition to growing cells, the progress of PG synthesis is followed by measuring the incorporation of new PG polymers into the matrix. The production of turnover products by LT 
enzymes can also be monitored following [3H]-mDAP labeling. The labeled GlcNAc-

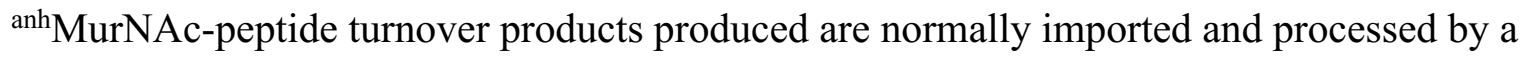
number of enzymes in the cytoplasm for recycling ${ }^{55}$. For our experiments, we used a strain lacking the recycling amidase AmpD to trap PG turnover products as cytoplasmic ${ }^{\text {anh MurNAc- }}$ peptides.

As in our previous experiments ${ }^{16}$, we focused our measurements on the cell elongation phase of PG synthesis by blocking cytokinetic ring assembly via induction of the FtsZ antagonist SulA thirty minutes prior to the addition of antibiotic. After five additional minutes of growth in the presence or absence of cefsulodin, $[3 \mathrm{H}]$-mDAP was added to the medium and growth was continued for 10 minutes (1/10th of a mass doubling). Cells were then harvested by centrifugation and extracted with hot $\left(90^{\circ} \mathrm{C}\right)$ water. Following centrifugation of the extract, soluble compounds (PG precursors and degradation products) remained in the supernatant and were separated and quantified by HPLC and radiodetection ${ }^{16}$. Label incorporated into the PG matrix was quantified as radioactivity released from the pellet by lysozyme. Importantly, because of the short labeling period, our measurements monitored the synthesis and degradation of nascent PG as opposed to that of bulk material in the mature matrix.

As observed previously ${ }^{16}$, cells with a full complement of LTs were capable of incorporating label into PG with or without cefsulodin treatment (Figure 2.14). However, cefsulodin induced a substantial increase in the level of turnover products detected. Inactivation of Slt did not affect the total level of label incorporation into PG, but turnover in the presence and absence of cefsulodin was reduced by approximately $50 \%$ (Figure 2.14). Conversely, inactivation of MltG had little effect on turnover, but total label incorporation into PG increased approximately $20 \%$ with or without cefsulodin treatment. The most profound effects on PG 
synthesis and turnover were observed in cells lacking both Slt and MltG (Figure 2.14). Total label incorporation into PG was increased by $60 \%$ and turnover was virtually undetectable whether or not cells were treated with cefsulodin. We infer from these results that MltG is capable of processing nascent PG in vivo, and that its activity along with that of Slt can somehow antagonize or slow the incorporation of new material into the PG matrix (see Discussion).

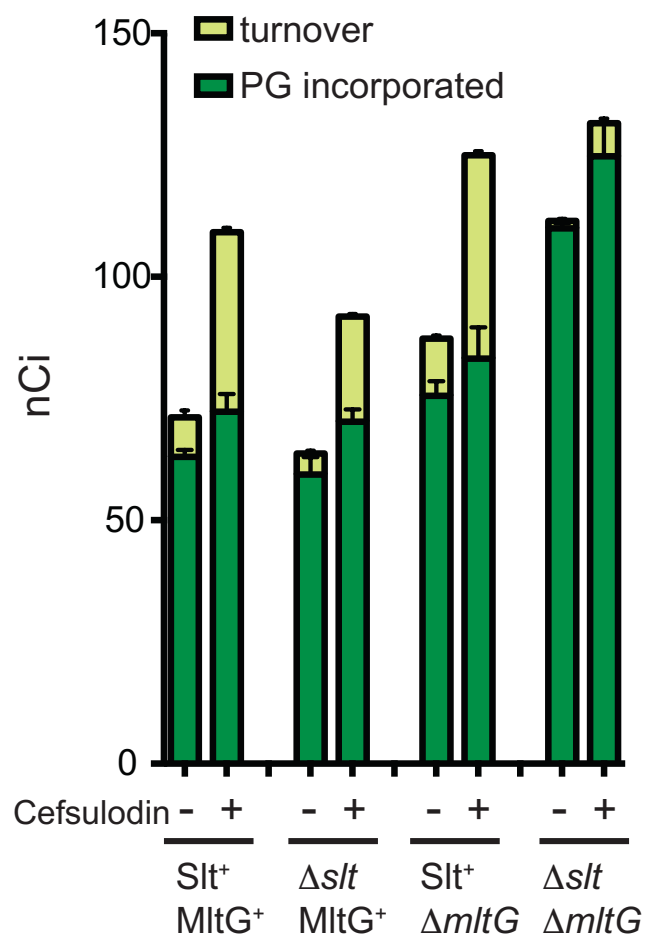

Figure 2.14. Nascent PG degradation by SIt and MltG following cefsulodin treatment. Cells of TU278 [ $\triangle$ lys $A \Delta a m p D]$ or its indicated derivatives were pulse labeled with $\left[{ }^{3} \mathrm{H}\right]-\mathrm{mDAP}$ with or without prior treatment with cefsulodin $(100 \mu \mathrm{g} / \mathrm{ml})$. Soluble metabolites were then extracted with hot water, separated by HPLC, and turnover products were quantified. Radiolabel incorporation into PG was determined by digesting the pellets resulting from the hot water extraction with lysozyme and quantifying the amount of label released into the supernatant by scintillation counting. Results are the average of three independent experiments with the error bars representing the standard deviation. See text and Material and Methods for experimental details. 


\subsection{DISCUSSION}

\section{PG glycan strand length and morphogenesis}

Bacterial cells come in a wide variety of shapes and sizes, and many bacteria also change their morphology in response to environmental conditions ${ }^{57}$. Because the PG matrix is the major determinant of bacterial cell shape, it is the collective activities of the various PG biogenesis factors that ultimately define the morphology of a given organism. Alterations of these activities are also undoubtably at the heart of any observed morphological transition. However, despite many years of study, the molecular mechanisms driving cell morphogenesis and morphogenic changes are still relatively poorly understood. One potentially important parameter for defining the size and shape of a bacterium is the length of glycan polymers inserted into the PG matrix by the PG synthases. In support of this possibility, average length of glycan strands is known to vary among different bacterial species and within a given organism upon changes in growth conditions ${ }^{58,59}$. Moreover, modeling studies predict that glycan strand length is a major variable affecting cell width ${ }^{60}$. Although PG polymerases from different organisms synthesize glycan chains of distinct lengths in vitro ${ }^{61}$, it has remained unclear how average polymer length is controlled in vivo. Furthermore, without knowing what factors govern strand length in growing cells, perturbations to the length control process to gain an understanding of its role in bacterial growth and morphogenesis have not been possible.

\section{A potential role for MItG as a PG polymerization terminase}

In this report, we identify MltG as a novel LT in E. coli and present evidence that it plays a key role in glycan strand length determination. For many years, it has been known that glycan strands in the E. coli PG matrix are "capped" by ${ }^{a n h}$ MurNAc sugars, and that these ends are 
formed early during synthesis ${ }^{1}$. These observations have led to the proposal that one or more LT enzymes may cleave nascent PG strands to terminate their synthesis ${ }^{23,}{ }^{22}$. Because PG strands undergoing active polymerization by the PG synthases are elongated from the reducing (MurNAc) end at the inner membrane and crosslinked into the matrix at the non-reducing (GlcNAc) end ${ }^{62}$, a putative LT terminase must be able to cleave the glycan backbone within the growing chain (Figure 2.15). However, most LTs studied to date are exoglycosidases that cleave glycan strands from a terminus to release soluble disaccharide products containing an ${ }^{\text {anh }}$ MurNAc sugar ${ }^{14,45}$. MltG, on the other hand, released ${ }^{\text {anh }}$ MurNAc-containing oligomeric products from purified E. coli $\mathrm{PG}$, indicating that it has the endoglycosidase activity necessary to function as a terminase. The only other E. coli LT with robust endoglycosidase activity is MltE(EmtA), which can cleave both peptide-free and peptide-containing PG strands ${ }^{23,14,45}$. RlpA from $P$. aeruginosa also has endoglycosidase activity, but is specific for peptide-free glycan strands ${ }^{15}$. The $E$. coli RlpA protein is likely to display a similar activity and substrate preference. Both MltE and RlpA, along with all other LTs previously characterized in E. coli, are reported to be localized to the outer membrane ${ }^{14,15}$. Although an outer membrane localization for these enzymes does not necessarily preclude them from processing nascent PG to affect glycan strand length, MltG is more likely to do so given its unique location in the inner membrane where glycan polymerization is taking place. Accordingly, a mutant lacking five outer membrane LTs (MltA, MltB, MltC, MltD, and MltE) along with periplasmic Slt did not show a large decrease in the ${ }^{\text {anh }}$ MurNAc content of its $\mathrm{PG}^{22}$. Conversely, the loss of MltG function alone resulted in a significant (ca. 25\%) drop in these chain ends and a corresponding increase in average glycan strand length, suggesting a function for MltG in chain length determination. Notably, this change in polymer length did not result in an observable change in cell width or 
length, indicating that if glycan chain length plays a significant role in cell morphology, more drastic changes are needed to reveal its importance.

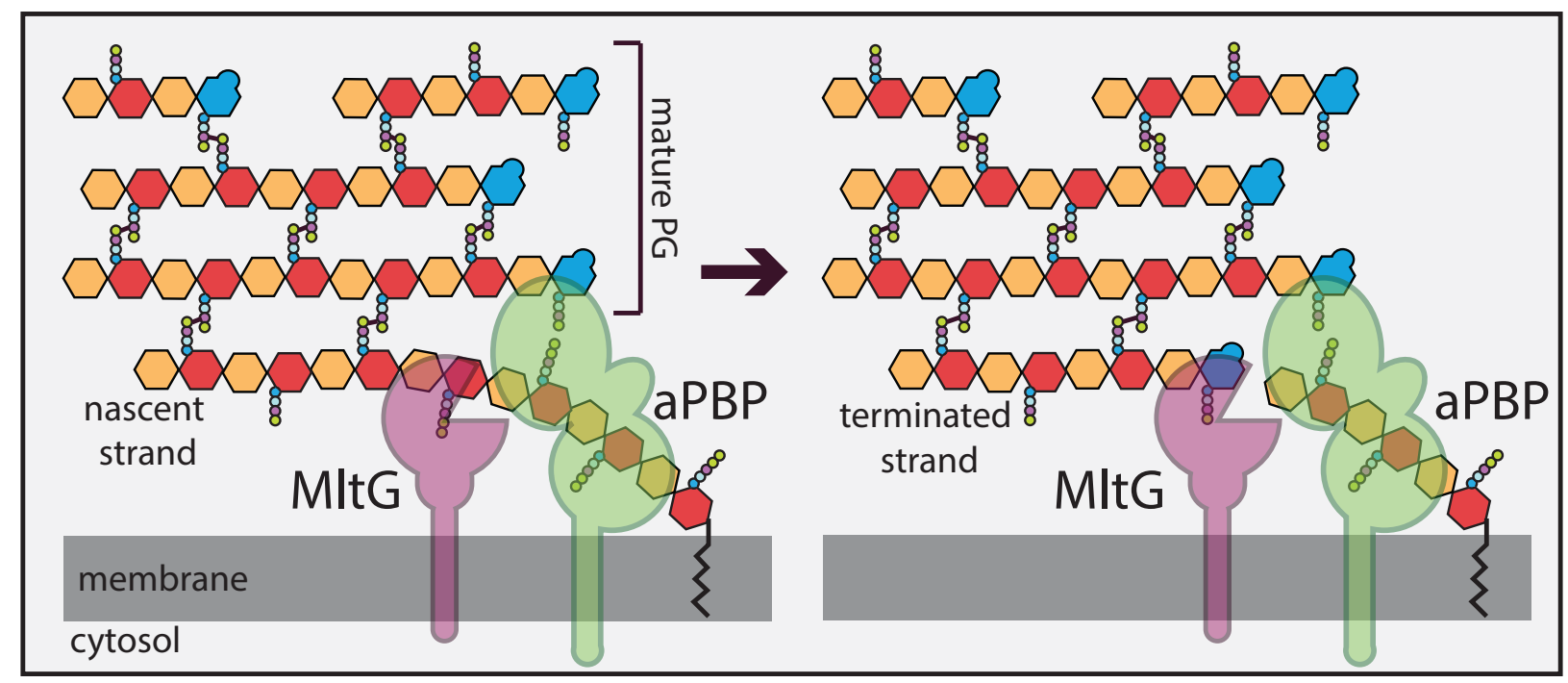

\section{Figure 2.15. Model for MltG functioning as a PG terminase.}

Shown is a model detailing the potential role of MltG as a terminase for PG polymerization. The PG matrix is drawn as in Figure 2.1 with GlcNAc in orange, MurNAc in red, and anh MurNAc in

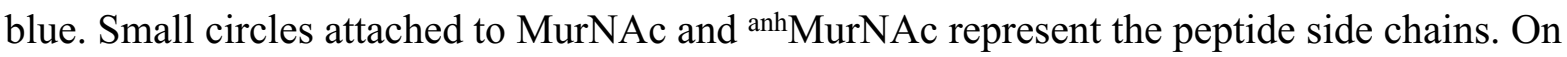
the left, an aPBP (green) is polymerizing a nascent glycan strand using its GT domain (circle closest to membrane). Although not shown, the polymers are extended by adding new dissacharides from the precursor lipid II to the growing chain at the membrane-proximal (MurNAc) end. We propose that MltG is recruited to the active polymerase via direct or indirect interactions. There, it cleaves the growing strand endolytically to terminate its elongation. It is not clear where along the strand MltG might cut. The site shown is merely to present the concept of a termination reaction. How much of the polymer remains associated with the polymerase post MltG cleavage and whether this portion of the chain can continue being elongated following cleavage also requires further investigation. 
Beyond its mere presence in the inner membrane, structural data provide additional support for nascent PG being the preferred substrate of MltG. The structure of an MltG-like protein from Listeria monocytogenes was recently deposited in the PDB (PDB: 4IIW) (Figure 2.16). This structure is closer to the full-length protein than the E. coli structure and includes more of the membrane-proximal domain (Figure 2.16). It reveals that this region has a LysM-like fold (Figure 2.16), a domain implicated in PG-binding ${ }^{63}$. MltG thus possesses a predicted PG binding domain quite close to the membrane surface where PG precursors are being polymerized into nascent material. Although the importance of this domain for MltG function is not known, it further implies that newly synthesized PG is the target of MltG cleavage activity. Moreover, BACTH results indicated that MltG forms a complex with the PBP1b polymerase, and radiolabeling analysis demonstrated that $\mathrm{MltG}$ is required for the turnover of nascent $\mathrm{PG}$ following cefsulodin treatment. Although other models remain possible, the sum of the data presented here makes a compelling case for MltG functioning to endolytically process nascent glycan strands undergoing polymerization to terminate their synthesis (Figure 2.15). 


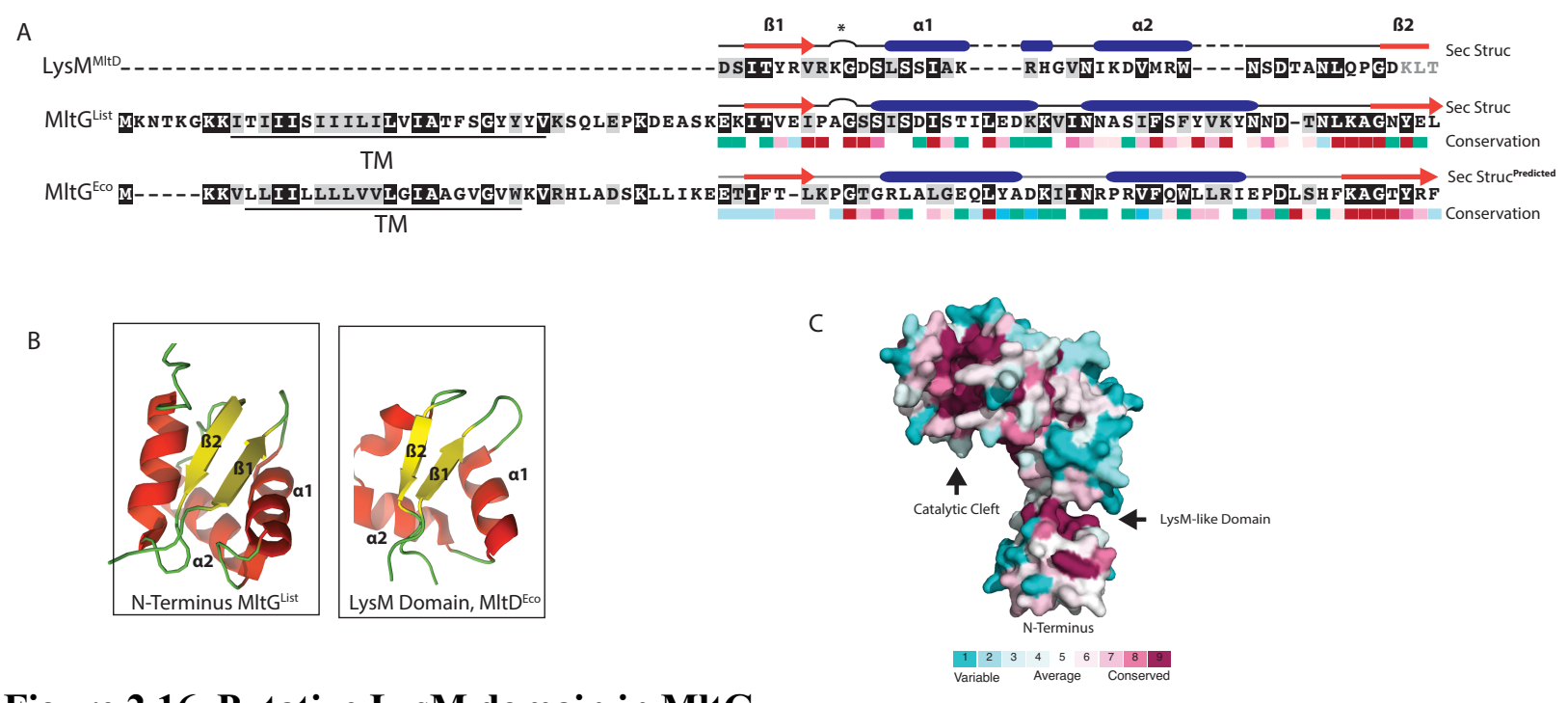

Figure 2.16. Putative LysM domain in MltG.

(A) The Phyre 2 server ${ }^{64}$ predicted the presence of a LysM-like domain near the N-terminus of $\mathrm{MltG}$, in a region that was not structurally characterized in E. coli MltG. However, the structure of the LysM-like region of MltG in Listeria monocytogenes has been solved (PDB: 4iiw).

Shown is the Phyre2 alignment of the canonical LysM domain from MltD with the predicted secondary structure and amino acid sequence of $\mathrm{MltG}^{E \text {. coli }}$ and the known secondary structure and sequence of $\mathrm{MltG}^{\text {Listeria }}$. The degree of conservation of each residue in this region of MltG is shown as a color coded box underneath each residue, determined using the Consurf server ${ }^{41}$. An asterisk (*) marks the putative ligand binding domain important for PG binding ${ }^{65}$. The color legend for conservation is shown in $(\mathbf{C})$.

(B) Cartoons of the crystal structures of the LysM domain from MltD (PDB:1E0G) and the predicted LysM-like domain of MltG from L. monocytogenes (PDB: 4iiw).

(C) Shown is a space-filling model of the MltG structure from L. monocytogenes (PDB 4iiw) colored to indicate amino acid conservation using ConSurf ${ }^{41}$. Note the high conservation of residues in the cleft-forming region that aligns with the known catalytic region of MltG from $E$. coli as well as the high degree of conservation near the $\mathrm{N}$-terminus in a cleft coinciding with the putative LysM-like domain. This LysM-like domain resides close to the predicted transmembrane region of MltG. 
MltG-like proteins are found in roughly $70 \%$ of sequenced bacteria. Notably, a major fraction of the species lacking MltG homologs are either coccal species or bacteria that lack cell walls, such as Mycoplasma. In contrast, almost all rod-shaped and ovococcal bacteria assessed encode MltG-like proteins, implying that MltG function may be particularly important to promote proper cell elongation. Consistent with this idea, work from Winkler and colleagues in preparation demonstrates that MltG is an essential component of the elongation (peripheral synthesis) machinery in S. pneumoniae (M. Winkler, personal communication). Additionally, although MltG inactivation does not result in a significant growth or morphological phenotype in E. coli, its homologs are reported to be essential in Helicobacter pylori and Mycobacterium tuberculosis ${ }^{66,67}$. Thus, the processing of PG by MltG-like factors may play a fundamental role in the morphogenesis of a major fraction of rod- and ovoid-shaped bacteria. Further work will be required to test this possibility and to determine why MltG-like factors are essential in some bacteria but not in others. Given the large number of LTs encoded by E. coli, it is likely that one or more of these additional enzymes can substitute for MltG to process nascent $\mathrm{PG}$, but we have yet to identify the (partially) redundant factor(s).

\section{The MltG-PBP1b connection}

Our studies have connected MltG with PBP1b in two different genetic assays: the multicopy lethal screen and the BACTH analysis. The reason why MltG overproduction is lethal in a strain defective for PBP1b is not known. Cells inactivated for PBP1b display defects in cell wall biogenesis and envelope integrity ${ }^{24,26,25}$, suggesting that PBP1b is one of the main PG synthases in the cell. Thus, the multicopy lethal phenotype of $m l t G$ in the absence of PBP $1 \mathrm{~b}$ may simply be due to the artificial elevation of PG cleavage activity in cells that are already 
experiencing difficulties building their wall. Alternatively, a more intimate regulatory connection between the two proteins is possible. Although it remains unclear whether the PBP1b-MltG association is mediated via direct protein-protein contacts or through their association with a common partner, the BACTH results suggest that the two proteins are in close physical proximity. Thus, it is possible that PBP1b associates with MltG to control its activity such that when PBP1b is inactivated, MltG becomes hyperactive and toxic to the cell when overproduced. If true, such a scenario would represent an interesting case of reciprocal control between a PG synthase and cleavage enzyme, with MltG determining the length of polymers produced by PBP1b, and PBP1b controlling the processing activity of MltG. Additional studies of MltG activity and its interaction partners should reveal the extent of cooperation between MltG and PBP1b in PG biogenesis and whether this interaction is specific or if MltG also associates with other PG polymerases in the cell.

\section{Degradation of nascent PG following cefsulodin treatment}

Cefsulodin specifically blocks the TP active sites of PBP1a and PBP1b ${ }^{56}$. Thus, in the presence of cefsulodin, the PG produced by these polymerases is likely to be uncrosslinked or at least poorly crosslinked. The increased turnover of nascent PG following cefsulodin treatment indicates that a significant portion of this poorly crosslinked material is targeted for degradation ${ }^{16}$. Furthermore, the increase in total radiolabel in the combined PG and turnover fractions indicates that PG polymerization is activated by TP inhibition, a phenomenon that has also been detected in vitro ${ }^{68}$. As observed previously ${ }^{16}$, inactivation of Slt led to a $50 \%$ drop in nascent PG turnover with or without cefsulodin treatment. MltG inactivation, on the other hand, had little effect on nascent PG turnover, but degradation was almost completely blocked in a 
strain lacking both Slt and MltG. From these results, we infer that Slt is the major LT that intervenes when aPBPs have problems coupling PG polymerization with crosslinking. This activity is consistent with the previously proposed quality control function for Slt ${ }^{16}$. Results from the $\Delta$ slt $\Delta m l t G$ mutant indicate that in the absence of Slt, MltG can step in and process the uncrosslinked or poorly crosslinked PG produced by the targeted aPBPs. This observation suggests the possibility that MltG may also function as a quality control or repair enzyme that can intervene to "rescue" damaged or malfunctioning PG polymerase complexes. However, unlike the $\Delta$ slt mutant, nascent PG processing following cefsulodin treatment is unaffected by MltG inactivation alone. Additionally, MltG is essential for the activity of the peripheral PG synthesis machinery of $S$. pneumoniae ${ }^{69}$. We therefore favor the idea that the primary function of MltG is to process nascent PG during the course of normal synthesis, but that it is also poised to cleave uncrosslinked strands produced by polymerases experiencing problems coupling GT and TP activities. Indeed, the proposed terminase activity of MltG may be in part controlled by the crosslinking activity of the PBPs. For example, MltG may be poised to cleave glycan strands when the synthesis machinery reaches a region of the PG matrix where acceptor peptides for crosslinking are sparse and uncrosslinked material begins to be produced.

In addition to their effects on nascent PG turnover, MltG and Slt also appear to slow or antagonize PG synthesis. Total PG synthesis during the pulse labeling increased in the absence of MltG and was further enhanced when Slt was also inactivated. The reason for the synthesis increase is not clear, but it suggests the possibility that LT cleavage of nascent PG strands by MltG may force the polymerases to "restart" PG polymerization, which may be rate-limiting relative to chain elongation. In the absence of MltG, the weak endo-LT activity detected for Slt ${ }^{45}$ may support some termination function in place of MltG. However, in the absence of both 
enzymes, termination activity may be greatly impaired such that the polymerases restart less often and therefore produce more material during the labeling pulse. Additional studies are required to test this and other possible models related to the interplay between PG cleavage by LT enzymes and synthesis by the polymerases. Nevertheless, the work reported here and in our previous report ${ }^{16}$ reveals that these two processes are more intimately connected than previously anticipated and may represent an additional control point in PG biogenesis amenable to antibiotic targeting.

\subsection{MATERIALS AND METHODS}

\section{Media, bacterial strains and plasmids:}

Cells were grown in LB [ $1 \%$ tryptone, $0.5 \%$ yeast extract, $0.5 \% \mathrm{NaCl}], 0.5 \mathrm{xLB}-0 \mathrm{~N}[0.5 \%$ tryptone, $0.25 \%$ yeast extract] or minimal M9 medium ${ }^{70}$ supplemented with $0.2 \%$ casamino acids and $0.2 \%$ maltose. Unless otherwise indicated, antibiotics were used at 25 (chloramphenicol; Cm), 25 (kanamycin; Kan), or 15 (ampicillin; Amp) $\mu \mathrm{g} / \mathrm{ml}$.

The bacterial strains used in this study are listed in Table 2.3. All E. coli strains used in the reported experiments are derivatives of MG1655 ${ }^{71}$. Plasmids used in this study are listed in Table 2.4. PCR was performed using KOD polymerase (Novagen) for cloning purposes and Taq DNA polymerase (NEB) for diagnostic purposes, both according to the manufacturer's instructions. Unless otherwise indicated, MG1655 chromosomal DNA was used as the template. Plasmid DNA and PCR fragments were purified using the Zyppy plasmid miniprep kit (Zymo Research) or the Qiaquick PCR purification kit (Qiagen), respectively. 
Table 2.3. Strains used in this study.

\begin{tabular}{|c|c|c|}
\hline Strain & Genotype $^{a}$ & Source/Reference ${ }^{b}$ \\
\hline ВТH101 & $\begin{array}{l}\text { cya-99 araD139 galE15 galK16 rpsL1 } \\
\left(\mathrm{Str}^{\mathrm{R}}\right), \text { hsdR2, mcrA1, mcrB1, relA1 }\end{array}$ & Euromedex \\
\hline BW25113 & $\begin{array}{l}\Delta(\text { araD-araB) } 567 \Delta l a c Z 4787(: \because r r n B-3) \\
\text { rph-1 } \Delta(\text { rhaD-rhaB) } 568 \text { hsdR514 }\end{array}$ & 78 \\
\hline DH5 $\alpha$ & $\begin{array}{l}F-\text { hsdR17 deoR recAl endA1 phoA supE44 } \\
\text { thi-1 gyrA96 relA1 } \triangle(\text { lacZYA-argF)U169 } \\
\varphi 80 d l a c Z \Delta M 15\end{array}$ & Gibco BRL \\
\hline JW1083 & $\mathrm{BW} 25113 \Delta y c e G 761:: \mathrm{Kan}^{\mathrm{R}}$ & 78 \\
\hline MG1655 & $r p h-1$ ilvG rfb-50 & 71 \\
\hline Rosetta2( $\lambda$ DE3) & $\begin{array}{l}\mathrm{F}^{-} \text {ompT hsd } \mathrm{S}_{\mathrm{B}}\left(\mathrm{r}_{\mathrm{B}^{-}} \mathrm{m}_{\mathrm{B}}^{-}\right) \text {gal dcm }(\mathrm{DE} 3) \\
\text { pRARE2 }\left(\mathrm{Cm}^{\mathrm{R}}\right)\end{array}$ & Novagen \\
\hline HC419 & $\begin{array}{l}\text { TB28 } \Delta l y s A 763:: \text { frt } \Delta a m p D 728:: f r t \\
\Delta s l t:: \operatorname{Kan}^{\mathrm{R}}\end{array}$ & 16 \\
\hline NP140 & TB28 $\Delta \operatorname{lpp}$ & TU163/pCP20 \\
\hline RY8 & TB28 $\Delta m l t G:: \operatorname{Kan}^{\mathrm{R}}$ & P1(JW1083)xTB28 \\
\hline RY34 & $\mathrm{TB} 10 \Delta m l t G:: \mathrm{Cm}^{\mathrm{R}}$ & $\lambda$ Red \\
\hline RY35 & $\mathrm{TB} 28 \Delta m l t G:: \mathrm{Cm}^{\mathrm{R}}$ & $\mathrm{P} 1(\mathrm{RY} 34) \times \mathrm{TB} 28$ \\
\hline RY36 & TB28 $\Delta m l t G:: f r t$ & $\mathrm{RY} 35 / \mathrm{pCP} 20$ \\
\hline RY43 & $\begin{array}{l}\text { TB28 } \Delta l y s A 763:: \text { frt } \Delta a m p D 728:: \text { frt } \\
\Delta m l t G:: \mathrm{Cm}^{\mathrm{R}}\end{array}$ & P1(RY34) x TU278 \\
\hline RY44 & $\begin{array}{l}\text { TB28 } \Delta l y s A 763:: \text { frt } \Delta a m p D 728:: \text { frt } \Delta s l t:: \\
\operatorname{Kan}^{\mathrm{R}} \Delta m l t G:: \mathrm{Cm}^{\mathrm{R}}\end{array}$ & P1(JW1083) x TB28 \\
\hline TB10 & rph-1 ilvG rfb-50 $\lambda \Delta$ cro-bio nad::Tn10 & 79 \\
\hline TB28 & MG1655 $\Delta l a c I Z Y A:: f r t$ & 28 \\
\hline
\end{tabular}


Table 2.3 (Continued). Strains used in this study.

\begin{tabular}{lll}
\hline Strain & Genotype $^{\mathbf{a}}$ & Source/Reference $^{\mathbf{b}}$ \\
\hline TU122 & TB28 $\Delta p o n B(\operatorname{mrcB}):: f r t$ & 24 \\
TU163 & TB28 $\Delta l p p:: \mathrm{Kan}^{\mathrm{R}}$ & 74 \\
TU278 & TB28 $\Delta l y s A 763:: f r t \Delta a m p D 728:: f r t$ & 16 \\
\hline
\end{tabular}

a The $\mathrm{Kan}^{\mathrm{R}}$ and $\mathrm{Cm}^{\mathrm{R}}$ cassettes are flanked by frt sites for removal by FLP recombinase. An $f r t$ scar remains following removal of the cassette using FLP expressed from pCP20.

b Strain constructions by $\mathrm{P} 1$ transduction are described using the shorthand: $\mathrm{P} 1$ (donor) $\mathrm{x}$ recipient. Transductants were selected on LB Kan or Cam plates where appropriate. Strains resulting from the removal of frt-flanked antibiotic resistance gene using pCP20 are indicated as: Parental strain/pCP20. $\lambda$ Red indicates strains were constructed by recombineering (see Experimental Procedures for details). 
Table 2.4. Plasmids used in this study.

\begin{tabular}{|c|c|c|c|}
\hline Plasmid & Genotype $^{\mathrm{a}}$ & Origin & Source/Reference \\
\hline pCM6 & cat $\mathrm{P}_{\text {ara::empty }}$ & $\mathrm{p} 15 \mathrm{~A}$ & This study \\
\hline pCP20 & bla cat cI875 repA(Ts) $\mathrm{P}_{\mathrm{R}}:$ flp & pSC101 & 73 \\
\hline $\mathrm{pDY} 1$ & cat lacI ${ }^{q} \mathrm{P}_{\text {lac::ponB } \text { lacZ }}$ & mini-F & This study \\
\hline pInt-ts & bla cI875 repA(Ts) $\mathrm{P}_{\mathrm{R}}:: i n t^{\lambda}$ & pSC101 & 80 \\
\hline pMM47 & attHK022 bla lacI ${ }^{q} \mathrm{P}_{\text {lac:: }}$ :sspal-mcherry & $\mathrm{R} 6 \mathrm{~K}$ & 24 \\
\hline pNP8 & attHK022 bla lacI ${ }^{q} \mathrm{P}_{\text {lac }}:: m l t G$ & R6K & This study \\
\hline pNP9 & att $\mathrm{HK} 022$ bla lacI ${ }^{q} \mathrm{P}_{\mathrm{lac}}:: m l t G$-mcherry & R6K & This study \\
\hline pRY30 & cat $\mathrm{P}_{\mathrm{ara}}::$ MG1655 loci (1153098-1154818) & $\mathrm{p} 15 \mathrm{~A}$ & $\begin{array}{l}\text { Screen Isolate } \\
\text { Genomic coordinates } \\
\text { of insert are indicated }\end{array}$ \\
\hline pRY42 & cat $\operatorname{lac}^{q} \mathrm{P}_{\mathrm{lac}}:: m l t G$ & $\mathrm{pBR} / \mathrm{colE} 1$ & This study \\
\hline pRY47 & cat lacI $q$ P lac::empty & $\mathrm{pBR} / \mathrm{colE} 1$ & 16 \\
\hline pRY53 & cat $\mathrm{P}_{\text {ara }}:: m l t G$ & $\mathrm{p} 15 \mathrm{~A}$ & This study \\
\hline pRY 54 & bla lac ${ }^{q} \mathrm{P}_{\mathrm{T} 7}:: h-$ sumo-s-mltG & $\mathrm{pBR} / \mathrm{colE} 1$ & This study \\
\hline pRY62 & bla lacI ${ }^{q} \mathrm{P}_{\mathrm{T} 7}:$ h-sumo-s-mltG(E218Q) & $\mathrm{pBR} / \mathrm{colE} 1$ & This study \\
\hline pRY66 & cat lacI ${ }^{q} \mathrm{P}_{\text {lac: }:: m l t G}(S 138 C)$ & $\mathrm{pBR} / \mathrm{colE} 1$ & This study \\
\hline pRY68 & cat lacI ${ }^{q} \mathrm{P}_{\text {lac: }}:$ mltG $(A 19 C)$ & $\mathrm{pBR} / \mathrm{colE} 1$ & This study \\
\hline pRY70 & cat lacI ${ }^{q} \mathrm{P}_{\text {lac: }}: m l t G(K 3 C)$ & $\mathrm{pBR} / \mathrm{colE} 1$ & This study \\
\hline pRY77 & $\begin{array}{l}\text { att } \mathrm{HK} 022 \text { bla lacI }{ }^{q} \mathrm{P}_{\mathrm{lac}::}: \mathrm{ss} p a l\left(D^{+2} E^{+3}\right)- \\
\text { mcherry }\end{array}$ & $\mathrm{R} 6 \mathrm{~K}$ & This study \\
\hline
\end{tabular}


Table 2.4 (Continued). Plasmids used in this study.

\begin{tabular}{|c|c|c|c|}
\hline Plasmid & Genotype $^{a}$ & Origin & Source/Reference \\
\hline pRY78 & 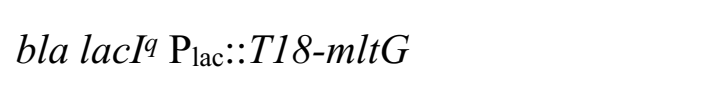 & $\mathrm{pBR} / \mathrm{colE} 1$ & This study \\
\hline pRY79 & aph $\mathrm{P}_{\text {lac }}:: T 25-m l t G$ & $\mathrm{p} 15 \mathrm{~A}$ & This study \\
\hline pRY86 & 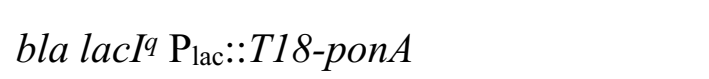 & $\mathrm{pBR} / \mathrm{colE} 1$ & This study \\
\hline pRY87 & aph $\mathrm{P}_{\text {lac }}:: T 25-p o n A$ & $\mathrm{p} 15 \mathrm{~A}$ & This study \\
\hline pRY88 & bla lacI ${ }^{q} \mathrm{P}_{\mathrm{lac}:: \text { T18-ponB }}$ & $\mathrm{pBR} /$ colE1 & This study \\
\hline pRY89 & aph $\mathrm{P}_{\text {lac }}:: T 25-p o n B$ & $\mathrm{p} 15 \mathrm{~A}$ & This study \\
\hline pTB102 & cat cI875 repA(Ts) $\mathrm{P}_{\mathrm{R}}::$ int ${ }^{H K 022}$ & pSC101 & 29 \\
\hline pTB145 & bla lacI ${ }^{q} \mathrm{P}_{T 7}:$ h-ulp1(403-621) & $\mathrm{pBR} / \mathrm{colE} 1$ & 75 \\
\hline pTB146 & bla lacI ${ }^{q} \mathrm{P}_{T 7}:$ h-sumo & $\mathrm{pBR} / \mathrm{colE} 1$ & 75 \\
\hline pTU136 & att $\mathrm{HK} 022$ bla lac ${ }^{q} \mathrm{P}_{\text {lac:: }:{ }^{\mathrm{ss}} d s b A-m \text { Cherry }}$ & R6K & 33 \\
\hline pUT18-zip & 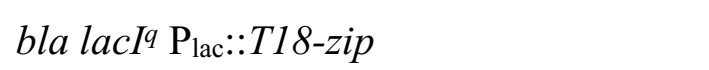 & $\mathrm{p} 15 \mathrm{~A}$ & Euromedex \\
\hline pKT25-zip & aph $\mathrm{P}_{\text {lac:::T25-zip }}$ & $\mathrm{pBR} / \mathrm{colE} 1$ & Euromedex \\
\hline
\end{tabular}

A 6xHis tag for purification is indicated by the letter $h .{ }^{s s} d s b A$ corresponds to the first 24 codons of $d s b A$ encoding its export signal. Plac, PT7, PR, and Para indicate the lactose, phage $\mathrm{T} 7, \lambda \mathrm{R}$ and arabinose promoters, respectively. Numbers in parenthesis indicate the codons included in the relevant clones. 


\section{Multicopy Library Preparation}

MG1655 genomic DNA was partially digested with Sau3AI. Digested DNA fragments were separated on an agarose gel, and fragments in the $1-4 \mathrm{~kb}$ range were excised and extracted from the matrix. The library of fragments was ligated with BamHI-digested pCM6 plasmid, a derivative of pBAD33 with only a single BamHI cleavage site in the multiple cloning region; the BamHI site in the arabinose promoter region was removed by site-directed mutagenesis. Ligation reactions were transformed into commercial electrocompetent DH5-alpha cells (New England Biolabs) yielding a library of approximately 30,000 transformants. The transformant colonies

were then slurried in LB broth. A portion of the slurry was used to prepare plasmid DNA using Qiagen miniprep kit, and the remainder was stored frozen at $-80^{\circ} \mathrm{C}$.

\section{Multicopy lethal screen}

Electrocompetent TU122/pDY1 [ $\triangle$ lacIZYA $\Delta$ ponB/Plac::ponB lacZ] cells were transformed with miniprepped DNA from the multicopy plasmid library. Transformants ( $>106$ to retain library complexity) were selected on LB media containing ampicillin, chloramphenicol, and IPTG $(250 \mu \mathrm{M})$. Amp was required to ensure that cells retained the mini-F plasmid whereas $\mathrm{Cm}$ selected for transformants that received a multicopy plasmid, respectively. Induction of ponB expression was controlled with IPTG $(250 \mu \mathrm{M})$. The library was collected and stored in aliquots at $-80^{\circ} \mathrm{C}$ in $\mathrm{LB}$ containing $15 \%$ glycerol.

For the screen, the TU122/pDY1 multicopy library was plated on LB plates supplemented with IPTG $(250 \mathrm{mM})$ and X-gal $(40 \mu \mathrm{g} / \mathrm{ml})$ to identify multicopy plasmids causing a lethal defect in the $\triangle p o n B$ background. For our screen, chloramphenicol $(10 \mu \mathrm{g} / \mathrm{mL})$ was also added to the screening plates to ensure retention of the multicopy plasmid. A total of 30,000 colonies were 
screened on media without arabinose, and 20,000 colonies were screened on media with $0.2 \%$ arabinose to induce Para on the pBAD33-derived vectors, yielding 6 solid-blue isolates that displayed varying degrees of IPTG-dependent growth. Multicopy plasmids were isolated from candidates of interest and their plasmid-insert junctions were sequenced to identify the chromosomal fragment contained within each plasmid.

\section{$\Delta m l t G:: C m R$ strain construction}

E. coli $m l t G$ resides upstream of essential genes ( $h o l B$ and $t m k$ ). To avoid potential effects on the holB and $t m k$ genes when inactivating $m l t G$, a $\triangle m l t G:: C m R$ allele was constructed using lambda red recombineering to replace the region between the 2 nd codon and the 25th codon of $m l t G$ with the $C m R$ cassette as described previously ${ }^{72}$. This effectively eliminates the transmembrane region of MltG encoded by the gene. Also, upon removing the $\mathrm{Cm}$ cassette with FLP recombinase, a frame-shift in mltG is created to disrupt its translation. The $C m R$ cassette was amplified from $\mathrm{pKD} 3{ }^{73}$ using the primers 5 '-AATATTTAGCCCCACTTTGTGAGCGCCC GAATTAGTCATGAATATCCTCCTTAGTTCCTA-3' and 5'-GTCTCTTCTTTGATAAGCA ATTTGCTGTCGGCAAGATGGCGTGTAGGCTGGAGCTGCTTC-3' (underlined regions correspond to homology to $\mathrm{pKD} 3$ necessary for PCR amplification of the Cm cassette, whereas the non-underlined regions correspond to homology to $m l t G$ necessary for recombineering). The resulting PCR product was purified and electroporated into strain TB10 as described previously

${ }^{28}$, and the recombinants were selected at $30^{\circ} \mathrm{C}$ on a LB plate containing $25 \mu \mathrm{g} / \mathrm{ml}$ chloramphenicol. 


\section{Protein Purification}

MltG(27-340), referred to as sMltG, and sMltG(E218Q) with a 6xHis-SUMO (H-SUMO) tag fused to their N-termini were purified from Rosetta 2( $\lambda \mathrm{DE} 3) / \mathrm{pRY} 54$ and Rosetta $2(\lambda \mathrm{DE} 3) / \mathrm{pRY} 62$, respectively, closely following procedures described previously ${ }^{74}$. The sequence of the affinity tag in all cases was MRGSHHHHHHMASG. After purification of the H-SUMO fusion protein by metal-affinity chromatography, the H-SUMO tag was removed using 6xHis-tagged SUMO protease (H-SP) ${ }^{75}$. Cleavage reactions were passed through Ni-NTA resin to remove free H-SUMO and H-SP, yielding a pure preparation of the desired protein. As a result of fusion construction, all proteins have an additional Ser residue at their N-termini.

Overnight cultures were grown at $37^{\circ} \mathrm{C}$ in LB supplemented with ampicillin $(15 \mu \mathrm{g} / \mathrm{ml})$ and glucose $(0.2 \%)$. The cultures were diluted 1:200 into 1L of LB supplemented with ampicillin (15 $\mu \mathrm{g} / \mathrm{ml})$ and glucose $(0.04 \%)$, and cells were grown at $37^{\circ} \mathrm{C}$ to an OD600 of 0.5 . IPTG was added to $0.5 \mathrm{mM}$, and the cultures were grown for an additional $3 \mathrm{hrs}$ at $37^{\circ} \mathrm{C}$. Cells were harvested by centrifugation, and the cell pellets were resuspended in $20 \mathrm{ml}$ of buffer A (50 mM Tris- $\mathrm{HCl} \mathrm{pH}$ $8.0,300 \mathrm{mM} \mathrm{NaCl}$ ) with $20 \mathrm{mM}$ imidazole and stored at $-80^{\circ} \mathrm{C}$ prior to use for protein purification. Cells were thawed and disrupted by passing them through a french pressure cell two times at 15,000 psi. Cell debris was pelleted by centrifugation at $17,000 \mathrm{x}$ g for $30 \mathrm{~min}$ at $4^{\circ} \mathrm{C}$.

The H-SUMO fusions were purified using $1 \mathrm{~mL}$ Ni-NTA agarose resin (Qiagen) according to the manufacturer's instructions. Resin was equilibrated in buffer A with $20 \mathrm{mM}$ imidazole. Thawed cell extract $(20 \mathrm{~mL})$ was combined with the equilibrated resin and incubated at $4^{\circ} \mathrm{C}$ with agitation to promote binding of the H-SUMO fusions. The resin was collected by centrifugation at $1000 \mathrm{x}$ for $3 \mathrm{~min}$ at $4^{\circ} \mathrm{C}$ to remove the non-conjugated material (wash 1), resuspended in buffer A with 20mM imidazole, and loaded onto a Poly-prep Chromatography Column (Biorad). 
After draining the supernatant, the column was washed with $4 \mathrm{ml}$ buffer A with $20 \mathrm{mM}$ imidazole (wash 2). The fusion proteins were then eluted with buffer A containing $300 \mathrm{mM}$ imidazole and peak fractions were collected. The purified H-SUMO fusions were incubated with a 1:1000 dilution of 6xHis-tagged SUMO protease (H-SP) and dialyzed overnight in buffer D (50mM Tris- $\mathrm{HCl} \mathrm{pH} 8.0,300 \mathrm{mM} \mathrm{NaCl}$, glycerol $10 \%$ ) at $4{ }^{\circ} \mathrm{C}^{74}$. The following morning, the protein preparation was passed over $1 \mathrm{ml} \mathrm{Ni-NTA}$ agarose resin (Qiagen). Untagged proteins eluted from the resin were dialyzed in $1 \mathrm{~L}$ of buffer $\mathrm{D}$ with $10 \%$ glycerol and stored at $-80^{\circ} \mathrm{C}$.

\section{Dye-release assay for PG hydrolysis}

Sacculi were prepared from strain TU163 $(\Delta l p p)$ and labeled with Remazol-Brilliant Blue as previously described ${ }^{74}$. RBB-labelled sacculi $(8 \mu \mathrm{l})$ were incubated at $37^{\circ} \mathrm{C}$ with purified sMltG $(4 \mu \mathrm{M})$, sMltG(E218Q) $(4 \mu \mathrm{M})$, lysozyme $(4 \mu \mathrm{M})$ or buffer D at $37^{\circ} \mathrm{C}$ in $40 \mu \mathrm{l}$ of $\mathrm{PBS}$ buffer (10 mM Na2HPO4, $2 \mathrm{mM} \mathrm{KH2PO4,} 137 \mathrm{mM} \mathrm{NaCl}$ and $2.7 \mathrm{mM} \mathrm{KCl,} \mathrm{pH} \mathrm{7.4)} \mathrm{for} \mathrm{the}$ indicated time points. After the indicated time periods, reactions were terminated by incubating them at $95^{\circ} \mathrm{C}$ for $5 \mathrm{~min}$. Following termination, all reactions were centrifuged at $16,000 \times \mathrm{g}$ for $20 \mathrm{~min}$ at room temperature. Supernatants were removed, and their absorbance was measured at $595 \mathrm{~nm}$.

\section{Cytological Assay for Membrane Localization}

Fluorescence microscopy was performed essentially as described previously ${ }^{33}$. The cytological assay for membrane localization of lipoproteins was performed as described

previously ${ }^{50}$. Specific growth conditions used for each experiment are given in figure legends.

Overnight cultures of the strains used in Figure 2.9 were back-diluted to an OD600 of 0.02 
in $5 \mathrm{~mL}$ M9-maltose supplemented with $100 \mu \mathrm{M}$ IPTG. Cells were grown at $37^{\circ} \mathrm{C}$ until they reached OD600 of 0.3, at which point they were harvested by centrifugation ( $5 \mathrm{~min}$ at $5000 \mathrm{x} \mathrm{g}$ ) and washed in M9-Maltose twice. The washed cells were resuspended in a final volume of $100 \mu 1$ M9-Maltose. $10 \mu 1$ of the cell resuspensions were transferred to a separate tube containing $10 \mu 1$ of 2x plasmolysis buffer [30\% sucrose, $50 \mathrm{mM}$ HEPES (pH 7.4), $40 \mathrm{mM}$ sodium azide], and immediately imaged using phase contrast and mCherry optics.

\section{Purification of PG Sacculi}

Exponential phase cultures of TB28 and its $\Delta m l t G$ derivative, RY36, were diluted 1:200 in $300 \mathrm{ml} \mathrm{LB}$ and grown at $37^{\circ} \mathrm{C}$ to OD600 0.6. Cells in the resulting cultures were pelleted, resuspended in $4 \mathrm{ml} \mathrm{PBS}$, added slowly to $4 \mathrm{ml}$ boiling $8 \%$ SDS (final [SDS] $=4 \%$ ), and boiled for $30 \mathrm{~min}$. Insoluble material after the boiling was collected by centrifugation $(125,000 \mathrm{x} \mathrm{g}, 1$ $\mathrm{hr}, 25^{\circ} \mathrm{C}$ ). Following several washes with water, the PG pellets were resuspended in $1 \mathrm{ml}$ PBS and incubated with $200 \mu \mathrm{g} / \mathrm{ml} \alpha$-amylase (Sigma) for $2 \mathrm{hr}$ at $37^{\circ} \mathrm{C}$. The sacculi were treated again with pronase (final concentration: $200 \mu \mathrm{g} / \mathrm{ml}$ ) overnight at $37^{\circ} \mathrm{C}$. The following morning, the sacculi were treated again with pronase (final concentration: $200 \mu \mathrm{g} / \mathrm{ml}$ ) for $1 \mathrm{hr}$ at $50^{\circ} \mathrm{C}$. Then, $10 \%$ SDS was added to each sample to a final concentration of $2 \%$, and the samples were heated at $95^{\circ} \mathrm{C}$ for $1 \mathrm{hr}$ to inactivate pronase. The de-proteinized sacculi were pelleted by centrifugation $\left(200,000 \mathrm{x} \mathrm{g}, 20 \mathrm{~min}, 25^{\circ} \mathrm{C}\right)$, washed several times with water to remove SDS, and resuspended in $200 \mu 1$ water containing $0.02 \%$ sodium azide.

\section{LC/MS Analysis of Mutanolysin-Digested Peptidoglycan}

Sacculi prepared from WT (TB28) and $\Delta m l t G$ (RY36) strains were digested with 
mutanolysin, and the resulting muropeptides were analyzed by LC/MS. Sacculi equivalent to the amount isolated from a $100 \mathrm{ml}$ culture (ca. $10^{10}$ cells) were digested overnight with $10 \mathrm{U}$ of mutanolysin (Sigma) in $50 \mathrm{mM}$ sodium phosphate buffer $(\mathrm{pH} 6.0)$ at $37^{\circ} \mathrm{C}$. The digests were centrifuged $\left(21,000 \mathrm{xg}\right.$ for $30 \mathrm{~min}$ at $\left.4^{\circ} \mathrm{C}\right)$, and the resulting supernatants were reduced by addition of $1 \mathrm{mg}$ of sodium borohydride in sodium borate buffer $(\mathrm{pH} 9.0)$ for $30 \mathrm{~min}$ at room temperature. The residual sodium borohydride was inactivated by addition of phosphoric acid to bring the sample $\mathrm{pH}$ to 6 . The samples were then centrifuged again as before to remove any particulate matter, lyophilized, resuspended in water, and analyzed using an Bruker Maxi Impact Q-TOF LC/MS system with Agilent 1290 HPLC outfitted with a Kinetex core shell C18 column (4.6 by $250 \mathrm{~mm}, 5 \mu \mathrm{m}$ resin size, $100 \AA$ pore size) with a Gemini C18 guard column. Muropeptides were eluted at a flow rate of $0.5 \mathrm{ml} / \mathrm{min}$ at a temperature of $50^{\circ} \mathrm{C}$ as follows: isocratic elution with $98 \%$ solvent $\mathrm{A}$ (water with $0.1 \%$ formic acid) and $2 \%$ solvent $\mathrm{B}$ (acetonitrile with $0.1 \%$ formic acid) for $5 \mathrm{~min}$, a 40 min linear gradient to $18 \% \mathrm{~B}$, a 15 min linear gradient to $90 \% \mathrm{~B}$, a 10 min linear gradient back to $2 \% \mathrm{~B}$, and an isocratic elution with $2 \%$ B for 5 min. For comparing the abundance of each muropeptide species among different samples, the representative ion species derived from each muropeptide was extracted and integrated from total ion chromatograms using Bruker Data Analysis software. The integrated ion counts were compared after normalizing them to the total ion counts for each sample.

\section{LC/MS Analysis of LT-Digested Peptidoglycan}

Sacculi prepared from WT (TB28) were digested with purified sMltG or purified Slt ${ }^{76}$ and the resulting muropeptides were analyzed by LC/MS. Sacculi equivalent to the amount isolated from a $100 \mathrm{ml}$ culture (ca. $10^{10}$ cells) were digested overnight with $4 \mu \mathrm{M}$ enzyme in $1 \mathrm{x}$ PBS 
(sMltG) or $25 \mathrm{mM}$ potassium acetate buffer, $\mathrm{pH} 4.5$ (Slt) at $37^{\circ} \mathrm{C}$. The digests were centrifuged $\left(21,000 \mathrm{x} \mathrm{g}\right.$ for $30 \mathrm{~min}$ at $\left.4^{\circ} \mathrm{C}\right)$, and the resulting supernatants were either reduced as described with mutanolysin-generated muropeptides or left unreduced. Analysis on LC/MS was performed as described for mutanolysin-generated muropeptides.

\section{Glycan strand analysis}

PG sacculi isolated from WT (TB28) and $\Delta m l t G$ (RY36) strains of $E$. coli were treated with purified E. coli $\mathrm{AmiD}^{76}$ for 48 hours at $37^{\circ} \mathrm{C}$ to release the glycan strands from the PG matrix. The digestion reaction was heat inactivated by incubating at $95^{\circ} \mathrm{C}$ for $10 \mathrm{~min}$. Uncleaved PG was pelleted, and the remaining supernatant was run directly on a Dionex HPLC system with a Nucleosil C1 8 column ( 4.6 by $250 \mathrm{~mm}, 5 \mu \mathrm{m}$ resin size, $300 \AA$ pore size) using the method described previously ${ }^{53}$.

\section{Scanning Cysteine Accessibility Mutagenesis (SCAM)}

Overnight cultures of RY8 [ $\Delta m l t G]$ carrying plasmids encoding IPTG-inducible variants of MltG with single cysteine substitutions (pRY66 - pRY70) were grown at $37^{\circ} \mathrm{C}$ in $\mathrm{LB}$ supplemented with chloramphenicol $(25 \mu \mathrm{g} / \mathrm{ml})$. The cultures were diluted 1:100 into $5 \mathrm{~mL}$ of LB supplemented with chloramphenicol $(25 \mu \mathrm{g} / \mathrm{ml})$, and cells were grown at $30^{\circ} \mathrm{C}$ to an $\mathrm{OD} 600$ of 0.5. IPTG was added to $100 \mu \mathrm{M}$ to induce expression of the MltG variants and the cultures were grown for an additional $1 \mathrm{hr}$ at $30^{\circ} \mathrm{C}$.

An aliquot of each culture $(1 \mathrm{ml})$ was normalized to OD600 of 1, washed twice with $1 \mathrm{ml}$ 1xPBS, and resuspended in a final volume of $90 \mu 1$ 1xPBS buffer. For the blocking step, tubes were set up containing $90 \mu 1$ of cell suspension and $10 \mu 1$ of either 1xPBS (untreated positive- 
and negative- label control tubes) or $100 \mathrm{mM}$ sodium (2-sulfonatoethyl) methanethiosulfonate $($ MTSES, Biotium), $[\mathrm{MTSES}]$ final $=10 \mathrm{mM})$. After rotating at room temperature for $10 \mathrm{~min}$, cells were washed three times with $1 \mathrm{ml} 1 \mathrm{x}$ PBS and resuspended in $100 \mu 1$ PEGylation buffer (60 mM Tris-HCl, pH 7.5, 1 mM EDTA, 1\% SDS, 10 M Urea) $)^{52}$. All samples except the negative-control tube were labelled with $5 \mathrm{mM}$ maleimide-polyethylene glycol (Mal-PEG, molecular weight $2 \mathrm{kDa}$, Nof Corporation Sunbright) for $30 \mathrm{~min}$ at $37^{\circ} \mathrm{C}$. To remove excess mPEG-mal and minimize its interference on the SDS-PAGE gel, each sample was TCAprecipitated by incubating with $10 \%$ TCA on ice for $1 \mathrm{hr}$, harvesting the protein pellet, and washing with $600 \mu 1$ ice-cold acetone. After letting the samples sit on ice with acetone for 20 min, the protein pellet was collected and resuspended in $100 \mu 1$ PEGylation buffer. The labelling of each sample was assessed using SDS-PAGE followed by immunoblotting with anti-MltG antibody.

\section{Immunoblotting}

Polyclonal rabbit antiserum was raised against purified sMltG by Covance Custom Immunology according to their standard protocol. The resulting anti-MltG antibodies were affinity purified using sMltG coupled to AminoLink resin (Pierce) using the standard protocol. For immunoblots, strains were grown as described in the figure legends. At the designated times, cells were harvested and whole-cell extracts were prepared as described previously ${ }^{77}$. The protein concentration of each extract was determined using the non-interfering protein assay (Genotech) according to the manufacturer's instructions. Protein concentrations were normalized between extracts, the samples were combined with 2x Laemmeli sample buffer with $10 \%$ Bmerceptoethanol, and the indicated amount of total protein from each extract was separated on a 
12\% SDS-PAGE gel (with 4\% stacking gel). Proteins were transferred to a PVDF membrane (Whatman) and the membrane was blocked with Rapid-Block (Amresco) for 5 minutes. The membrane was incubated with primary anti-MltG antibodies diluted in Rapid-Block $(1: 10,000)$ for 1 hour with gentile agitation at room temperature. The primary antibody solution was then removed and the membrane was quickly rinsed with TBS-T (10mM Tris-HCl pH 7.5, 100mM $\mathrm{NaCl}, 0.1 \%$ Tween-20) and thoroughly washed three times with $25 \mathrm{ml}$ TBS-T for 10 minutes each wash. Following the final wash, the membrane was incubated with secondary goat antirabbit antibodies conjugated to horseradish peroxidase (Rockland) diluted 1:35,000 in RapidBlock for 1 hour with gentle agitation at room temperature. After this incubation period, the secondary antibody solution was discarded and the membrane was again quickly rinsed with TBS-T and then thoroughly washed an additional three times with $25 \mathrm{ml}$ TBS-T for 10 minutes each. The blot was developed using the Super Signal West Pico system (Pierce) according to the manufacturer's protocol. Chemiluminescence was detected using a BioRad Chemidoc system.

\section{Bacterial two hybrid analysis}

BACTH plasmids were co-transformed into BTH101 (cya-99). Transformants were grown overnight in LB supplemented with $50 \mu \mathrm{g} / \mathrm{ml}$ ampicillin, $25 \mu \mathrm{g} / \mathrm{ml}$ kanamycin, and $500 \mu \mathrm{M}$

IPTG. Spots of $5 \mu 1$ of stationary-phase cultures were then spotted onto LB agar plates containing $50 \mu \mathrm{g} / \mathrm{ml}$ ampicillin, $25 \mu \mathrm{g} / \mathrm{ml}$ kanamycin, and $500 \mu \mathrm{M}$ IPTG with $40 \mu \mathrm{g} / \mathrm{mL}$ X-gal. Plates were imaged after incubation at $30^{\circ} \mathrm{C}$ for $24-48$ hours.

\section{Measurement of PG synthesis and turnover}

The synthesis and turnover of nascent PG was monitored by using $\triangle l y s A \Delta a m p D$ strains 
essentially as described previously $\left({ }^{55}, 16\right)$. Cells of the relevant strains were grown overnight in M9-glycerol medium supplemented with $0.2 \%$ casamino acids. The resulting cultures were then diluted to an OD600 of 0.04 in the same medium and grown to an OD600 between 0.3-0.4 at $30^{\circ} \mathrm{C}$. Cell division was then blocked by the induction of sulA from a chromosomally integrated plasmid (pHC859) for 30 min before drug treatment. The cultures were then treated with the cefsulodin $(100 \mu \mathrm{g} / \mathrm{ml})$ for 5 min after adjusting the culture OD600 to 0.3. Following drug treatment, $[3 \mathrm{H}]$-mDAP $(1 \mu \mathrm{Ci})$ was added to $1 \mathrm{~mL}$ of each culture for $10 \mathrm{~min}(1 / 10$ of the doubling time) to label the newly synthesized PG and its turnover products. After the labeling, cells were pelleted, resuspended in $0.7 \mathrm{ml}$ water, and heated at $90^{\circ} \mathrm{C}$ for 30 min to extract watersoluble compounds. After the hot water extraction, insoluble material was pelleted by ultracentrifugation $\left(200,000 \mathrm{xg}\right.$ for $20 \mathrm{~min}$ at $\left.4^{\circ} \mathrm{C}\right)$. The resulting supernatant was then removed, lyophilized, and resuspended in $0.1 \%$ formic acid for HPLC analysis. To determine [3H]-mDAP incorporated into the PG matrix, the pellet fraction was washed with $0.7 \mathrm{~mL}$ buffer A (20 mM Tris- $\mathrm{HCl}, \mathrm{pH} 7.4,25 \mathrm{mM} \mathrm{NaCl}$ ) and resuspended in $0.5 \mathrm{~mL}$ buffer A containing $0.25 \mathrm{mg}$ lysozyme. The suspensions were incubated overnight at $37^{\circ} \mathrm{C}$. Insoluble material was then pelleted by centrifugation $\left(21,000 \times \mathrm{g}\right.$ for $30 \mathrm{~min}$ at $\left.4^{\circ} \mathrm{C}\right)$, and the resulting supernatant was mixed with $10 \mathrm{~mL}$ EcoLite (MP biomedicals) scintillation fluid and quantified in Microbeta Trilux 1450 liquid scintillation counter (Perkin-Elmer).

\section{Conservation Analysis}

A previously constructed phylogenic tree containing a diverse representation of bacteria was used to select the genomes for analysis ${ }^{46}$. The genome set was assessed for the presence or 
absence of proteins containing signature Pfams (Finn et al., 2013) that serve as the minimal domain constituent for each major family of LTs.

\section{Plasmid Construction}

pCM6

The plasmid pCM6 [cat Para::empty] was constructed as follows. The pBAD33 vector ${ }^{30}$ was modified by Quick-Change site-directed mutagenesis to remove the BamHI site located in the arabinose promoter region using the primers 5'-ATCCATAAGATTAGCGAATCCTACCTGA CGC-3' and 5'-GCGTCAGGTAGGATTCGCTAATCTTATGGAT-3' according to standard procedures.

pDY1

The plasmid pDY1 [bla lacIq Plac::ponB lacZ] was constructed as follows. The primers

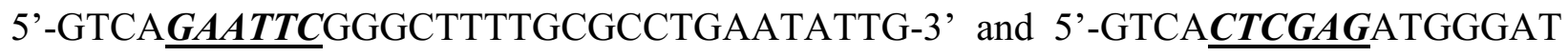
GTTATTTTACCGGATGGC-3' were used to amplify ponB from pTU110 ${ }^{24}$. The resulting PCR product was purified, digested with EcoRI and XhoI, and ligated into $\mathrm{pRC} 7{ }^{81}$ digested with EcoRI and SalI.

$\mathrm{pNP8}$

The plasmid pNP8 [attHK022 bla lacIq Plac::mltG] was constructed as follows. The primers 5'-GTACAAGCTTTTACTGCGCATTTTTTTCCTTAAGCACTTTC-3' and 5'-GTAC

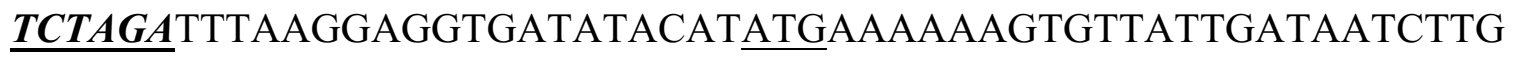
TTATTGCTGGTG-3' were used to amplify mltG from E. coli MG1655 genomic DNA. The 
resulting PCR product was purified, digested with XbaI and HindIII, and ligated into similarly digested pTB225 ${ }^{33}$.

pNP9

The plasmid pNP9 [attHK022 bla lacIq Plac::mltG-mcherry] was constructed as follows. The

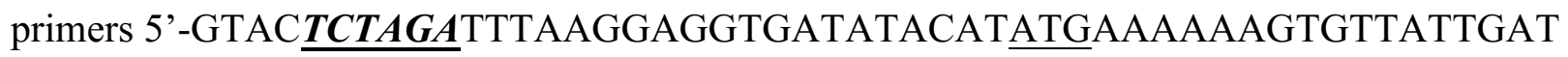

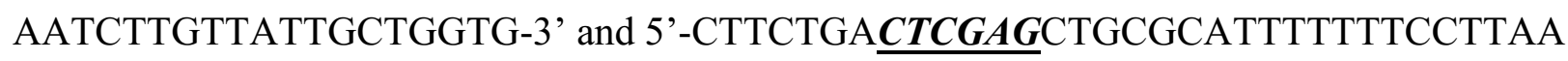
GCACTTTCAG-3' were used to amplify $m l t G$ from E. coli MG1655 genomic DNA. The resulting PCR product was purified, digested with XbaI and XhoI, and ligated into similarly digested $\mathrm{pNP} 3{ }^{82}$.

$\mathrm{pRY} 42$

The plasmid pRY42 [cat lacIq Plac::mltG] was constructed as follows. The $m l t G$ containing XbaI/HindIII fragment of pNP8 [attHK022 bla lacIq Plac::mltG] was used to replace the corresponding fragment of pTU272 [cat lacIq Plac::slmA].

pRY52

The plasmid pRY52 [cat Para::mltG(E218Q)] was constructed as follows. The internal primers 5'-TGGCATCAATTATCGAAAAACAAACCGCCGTTGCCAGTGAACGC-3' , 5'-GCGTTC ACTGGCAACGGCGGTTTGTTTTTCGATAATTGATGCCA-3' and the external primers 5'GTAC $\underline{\boldsymbol{T C T} \boldsymbol{A G} \boldsymbol{A}}$ TTTAAGGAGGTGATATACATATGAAAAAAGTGTTATTGATAATCTTG

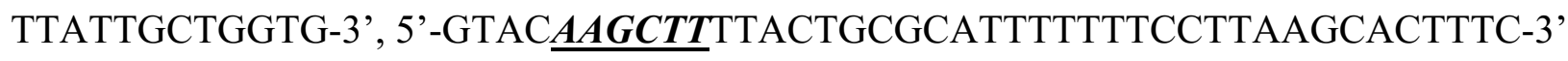
were used in overlap extension PCR to amplify $m l t G$ from genomic DNA and to perform site 
directed mutagenesis to create the E218Q mutation in the mlt $G$ gene. The resulting PCR product was purified, digested with XbaI and HindIII, and ligated into similarly digested pTB285 ${ }^{74}$.

pRY53

The plasmid pRY53 [cat Para::mltG] was constructed as follows. The mlt $G$ containing XbaI/HindIII fragment of pNP8 [attHK022 bla lacIq Plac::mltG] was used to replace the corresponding fragment of pCM6.

pRY54

The plasmid pRY54 [bla lacIq PT7::h-sumo-s-mltG] was constructed as follows. The primers 5'-

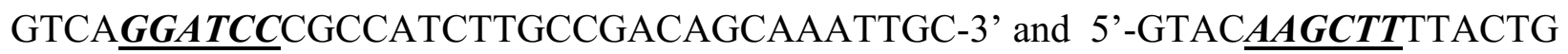
CGCATTTTTTTCCTTAAGCACTTTC-3' were used to amplify $m l t G$ from pNP8. The resulting PCR product was purified, digested with HindIII and BamHI-HF, and ligated into similarly digested pTD68 ${ }^{76}$.

pRY62

The plasmid pRY62 [bla lacIq PT7::h-sumo-s-mltG(E218Q)] was constructed as follows. The

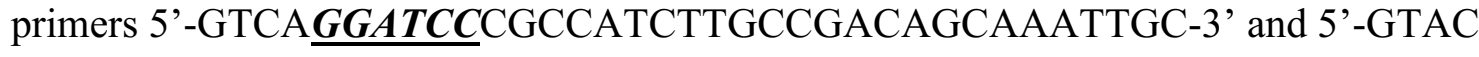

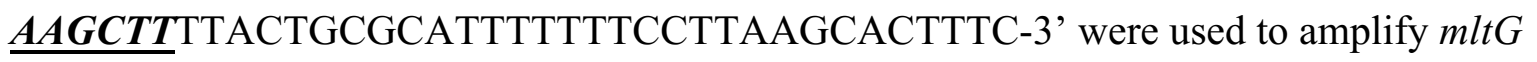
(E218Q)27-340 from pRY52. The resulting PCR product was purified, digested with HindIII/BamHI-HF, and ligated into pTD68 ${ }^{76}$. 
pRY66

The plasmid pRY66 [cat lacIq Plac::mltG (S138C)] was constructed as follows. The internal primers 5'-CGTATATCAAGCATACGCTGTGCGATGATAAGTACGCCACC-3', 5'GGTGGCGTACTTATCATCGCACAGCGTATGCTTGATATACG-3' and external primers 5'GTAC $\underline{\boldsymbol{T C T} \boldsymbol{A} \boldsymbol{G} \boldsymbol{A}}$ TTTAAGGAGGTGATATACATATGAAAAAAGTGTTATTGATAATCTTG

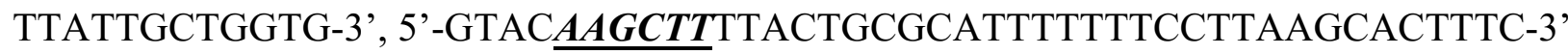
were used in overlap extension PCR to amplify $m l t G$ from genomic DNA and to perform site directed mutagenesis to create the $\mathrm{S} 138 \mathrm{C}$ mutation in the $m l t G$ gene. The resulting PCR product was purified, digested with $\mathrm{XbaI}$ and HindIII, and ligated into similarly digested pTU272 [cat lacIq Plac::slmA].

pRY68

The plasmid pRY68 [cat lacIq Plac::mltG (A19C)] was constructed as follows. The internal primers 5'-TGGTGGTACTGGGTATCGCCTGTGGTGTGGGCGTCTGGAAGG-3', 5'CCTTCCAGACGCCCACACCACAGGCGATACCCAGTACCACCA-3' and the external primers 5'-GTAC $\underline{\boldsymbol{T C T} \boldsymbol{A} \boldsymbol{G} \boldsymbol{A}}$ TTTAAGGAGGTGATATACATATGAAAAAAGTGTTATTGA

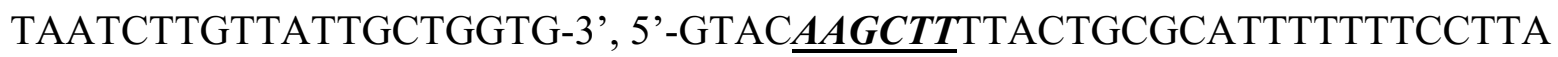
AGCACTTTC-3' were used in overlap extension PCR to amplify $m l t G$ from genomic DNA and to perform site directed mutagenesis to create the A19C mutation in the $m l t G$ gene. The resulting PCR product was purified, digested with XbaI and HindIII, and ligated into similarly digested pTU272 [cat lacIq Plac::slmA]. 
pRY70

The plasmid pRY70 [cat lacIq Plac::mltG $(K 3 C)$ ] was constructed as follows. The internal primers 5'-GAGGTGATATACATATGAAATGCGTGTTATTGATAATCTTGTT-3', 5'AACAAGATTATCAATA ACACGCATTTCATATGTATATCACCTC-3' and the external primers 5'-GTAC $\underline{\boldsymbol{T C T} \boldsymbol{A G} \boldsymbol{A}}$ TTTAAGGAGGTGATATACATATGAAAAAAGTGTTATTG

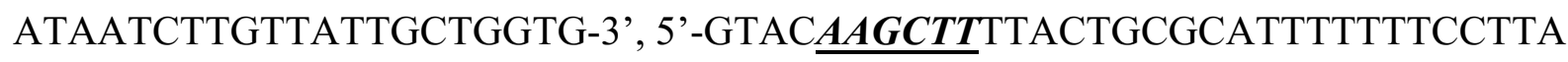
AGCACTTTC-3' were used in overlap extension PCR to amplify $m l t G$ from genomic DNA and to perform site directed mutagenesis to create the $\mathrm{K} 3 \mathrm{C}$ mutation in the $m l t G$ gene. The resulting PCR product was purified, digested with XbaI and HindIII, and ligated into similarly digested pTU272 [cat lacIq Plac::slmA].

pRY77

The plasmid pRY77 [attHK022 bla lacIq Plac::sspal(D+2 E+3)-mcherry] was constructed as follows. The gene fragment encoding $\operatorname{sspal}(D+2 E+3)$ GTAC $\underline{\boldsymbol{T C T A} \boldsymbol{A} \boldsymbol{A} A A A T A A T T T T G T T T A A}$ CTTTAAGAAGGAGATATACATATGCAACTGAACAAAGTGCTGAAAGGGCTGATGAT

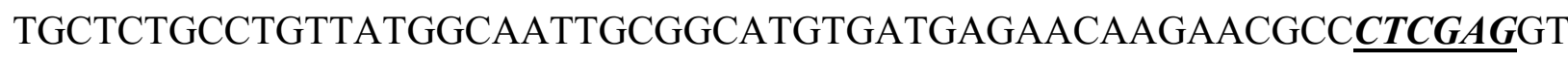
AC was synthesized as a gBlock by Integrated DNA Technologies (IDT). This gBlock was digested with XbaI and XhoI, and ligated into similarly digested pMM46 ${ }^{24}$.

pRY78

The plasmid pRY78 [bla lacIq Plac::T18-mltG] was constructed as follows. The primers 5'-

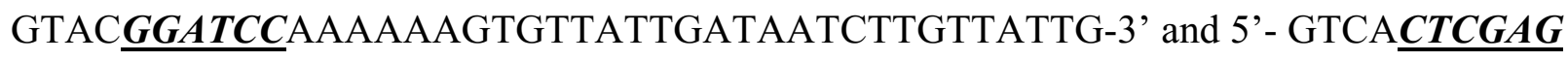
TTACTGCGCATTTTTTTCCTTAAGC-3' were used to amplify $m l t G$ from pRY42. The 
resulting PCR product was purified, digested with BamHI-HF / XhoI, and ligated into similarly digested pTU234 [bla lacIq Plac::T18-amiA].

pRY79

The plasmid pRY79 [aph Plac::T25-mltG] was constructed as follows. The primers 5'-

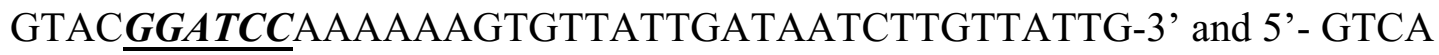
$\underline{\boldsymbol{C} \boldsymbol{T} \boldsymbol{G} \boldsymbol{A} \boldsymbol{G}}$ TTACTGCGCATTTTTTTCCTTAAGC-3' were used to amplify $m l t G$ from pRY42. The resulting PCR product was purified, digested with BamHI-HF / XhoI, and ligated into similarly digested pTU235 [aph Plac::T25-amiA].

pRY86

The plasmid pRY86 [bla lacIq Plac::T18-ponA] was constructed in several steps as follows. The primers 5'-AGGTCGACTCTAGAA $\underline{\boldsymbol{G G A T C C}}$ AAGTTCGTAAAGTATTTTTTGATC-3' and 5'-GAGCTCAAGCTTATTACTCAGAACAATTCCTGTGC-3' were used to amplify ponA from E. coli MG1655 genomic DNA. Using isothermal assembly, the resulting PCR fragment was incorporated into the corresponding fragment of BamHI-HF/XhoI digested pRY78.

\section{pRY87}

The plasmid pRY87 [aph Plac::T25-ponA] was constructed as follows. First, the primers 5'-

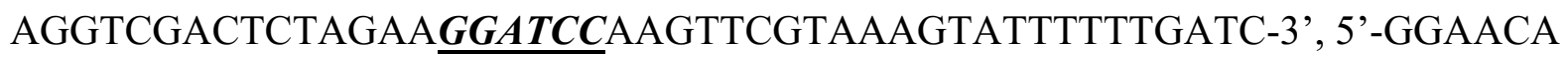
ATTCCTGTGCCTCGCCATTATC-3' were used to amplify ponA from E. coli MG1655 genomic DNA. In a second PCR reaction, the primers 5'-AGGTCGACTCTAGAA $\underline{\boldsymbol{G} \boldsymbol{G} \boldsymbol{A} \boldsymbol{T C C A A}}$

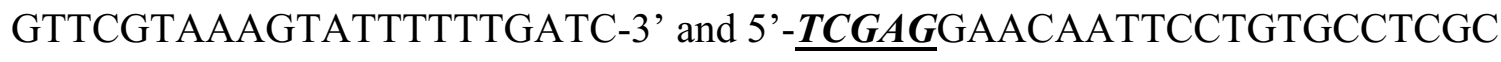


CATTATC-3' were used to amplify ponA with a 3' XhoI restriction site. ponA contains an internal Xhol restriction site, so instead of digesting with Xhol, the two PCR products were mixed and annealed to create an overhang similar to that obtained with XhoI digestion $(\mathrm{C} / \underline{T C G A G})$. The resulting product was purified, digested with BamHI-HF, and ligated into BamHI-HF/XhoI digested pRY79.

pRY88

The plasmid pRY88 [bla lacIq Plac::T18-ponB] was constructed as follows. The primers 5'-

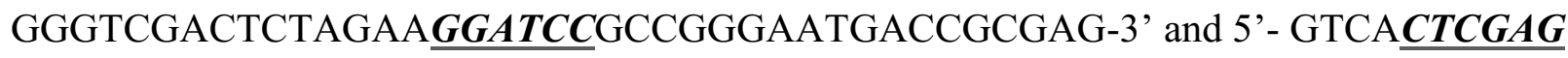
TTAATTACTACCAAACATATCCTTGATCCAACC-3' were used to amplify ponB from E. coli MG1655 genomic DNA. The resulting PCR product was purified, digested with BamHI-HF and XhoI, and ligated into similarly digested pTU134 [bla lacIq Plac::T18-amiA] a derivative of pHC364 ${ }^{75}$.

pRY89

The plasmid pRY89 [aph Plac::T25-ponB] was constructed as follows. The primers 5'-

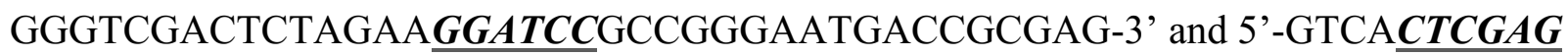
TTAATTACTACCAAACATATCCTTGATCCAACC-3' were used to amplify ponB from E. coli MG1655 genomic DNA. The resulting PCR product was purified, digested with BamHI-HF and XhoI, and ligated into similarly digested pTU135 [aph Plac::T25-amiA] a derivative of pKT25 [aph Plac::T25-zip] ${ }^{54}$. 
pTU162

The plasmid pTU162 [attHK022 bla lacIq Plac::slt] was constructed as follows. The primers 5'-

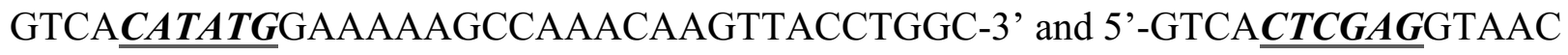
GACGTCCCCATTCCGTG-3' were used to amplify slt from E. coli MG1655 genomic DNA. The resulting PCR product was purified, digested with NdeI and XhoI, and ligated into similarly digested pTU148 [attHK022 bla lacIq Plac::ssdsbA-mCherry] ${ }^{82}$.

\section{Acknowledgements}

The authors would like to thank all members of the Bernhardt and Rudner labs for advice and helpful discussions. We would also like to thank Dana Boyd for initial help with the bioinformatic analysis of MltG conservation. We are also grateful to Suzanne Walker and her laboratory for help with LC/MS. Muropeptide analysis was performed using LC/MS at the Harvard Small Molecular Mass Spectrometry Core with the help of Sunia Trauger and Gary Byrd. Special thanks to Malcolm Winkler for communicating results prior to publication. This work was supported by the National Institute of Allergy and Infections Diseases of the National Institutes of Health (R01AI083365). R.Y. was supported in part by predoctoral fellowships from the National Science Foundation (DGE0946799, DGE0644491, DGE1144152), the ASM Robert D. Watkins Fellowship, and the National Institutes of Health (F31AI110046). 


\subsection{REFERENCES}

1. Höltje, J. V. Growth of the stress-bearing and shape-maintaining murein sacculus of Escherichia coli. Microbiology and molecular biology reviews : MMBR 62, 181-203 (1998).

2. Vollmer, W. \& Seligman, S. J. Architecture of peptidoglycan: more data and more models. Trends in microbiology 18, 59-66 (2010).

3. Taubes, G. The bacteria fight back. Science 321, 356-361 (2008).

4. McKenna, M. Antibiotic resistance: the last resort. Nature 499, 394-396 (2013).

5. $\quad$ Sauvage, E., Kerff, F. D. R., Terrak, M., Ayala, J. A. \& Charlier, P. The penicillinbinding proteins: structure and role in peptidoglycan biosynthesis. FEMS microbiology reviews 32, 234-258 (2008).

6. Tipper, D. J. \& Strominger, J. L. Mechanism of action of penicillins: a proposal based on their structural similarity to acyl-D-alanyl-D-alanine. Proceedings of the National Academy of Sciences 54,1133-1141 (1965).

7. Uehara, T. \& Bernhardt, T. G. More than just lysins: peptidoglycan hydrolases tailor the cell wall. Current opinion in microbiology 14, 698-703 (2011).

8. Bisicchia, P. et al. The essential YycFG two-component system controls cell wall metabolism in Bacillus subtilis. Mol. Microbiol. 65, 180-200 (2007).

9. Hashimoto, M., Ooiwa, S. \& Sekiguchi, J. Synthetic lethality of the lytE cwlO genotype in Bacillus subtilis is caused by lack of D,L-endopeptidase activity at the lateral cell wall. Journal of bacteriology 194, 796-803 (2012).

10. Singh, S. K., SaiSree, L., Amrutha, R. N. \& Reddy, M. Three redundant murein endopeptidases catalyse an essential cleavage step in peptidoglycan synthesis of Escherichia coli K12. Mol. Microbiol. 86, 1036-1051 (2012).

11. Meisner, J. et al. FtsEX is required for CwlO peptidoglycan hydrolase activity during cell wall elongation in Bacillus subtilis. Mol. Microbiol. 89, 1069-1083 (2013).

12. Domínguez-Cuevas, P., Porcelli, I., Daniel, R. A. \& Errington, J. Differentiated roles for MreB-actin isologues and autolytic enzymes in Bacillus subtilismorphogenesis. Mol. Microbiol. 89, 1084-1098 (2013).

13. Heidrich, C. et al. Involvement of N-acetylmuramyl-L-alanine amidases in cell separation and antibiotic-induced autolysis of Escherichia coli. Mol. Microbiol. 41, 167178 (2001). 
14. van Heijenoort, J. Peptidoglycan Hydrolases of Escherichia coli. Microbiology and Molecular Biology Reviews 75, 636-663 (2011).

15. Jorgenson, M. A., Chen, Y., Yahashiri, A., Popham, D. L. \& Weiss, D. S. The bacterial septal ring protein RlpA is a lytic transglycosylase that contributes to rod shape and daughter cell separation in Pseudomonas aeruginosa. Mol. Microbiol. 93, 113-128 (2014).

16. Cho, H., Uehara, T. \& Bernhardt, T. G. Beta-lactam antibiotics induce a lethal malfunctioning of the bacterial cell wall synthesis machinery. Cell 159, 1300-1311 (2014).

17. Dijkstra, A. J. \& Keck, W. Peptidoglycan as a barrier to transenvelope transport. Journal of bacteriology 178, 5555 (1996).

18. Koraimann, G. Lytic transglycosylases in macromolecular transport systems of Gramnegative bacteria. Cellular and molecular life sciences: CMLS 60, 2371-2388 (2003).

19. Scheurwater, E. M. \& Burrows, L. L. Maintaining network security: how macromolecular structures cross the peptidoglycan layer. FEMS microbiology letters $\mathbf{3 1 8}$, 1-9 (2011).

20. Burman, L. G. \& Park, J. T. Changes in the composition of Escherichia coli murein as it ages during exponential growth. Journal of bacteriology 155, 447-453 (1983).

21. Glauner, B. \& al, E. Growth pattern of the murein sacculus of Escherichia coli. J. Biol. Chem. 265, 18988-18996 (1990).

22. Heidrich, C., Ursinus, A., Berger, J., Schwarz, H. \& Höltje, J. V. Effects of Multiple Deletions of Murein Hydrolases on Viability, Septum Cleavage, and Sensitivity to Large Toxic Molecules in Escherichia coli. Journal of bacteriology 184, 6093-6099 (2002).

23. Kraft, A., Templin, M. \& ltje, J. H. O. Membrane-bound lytic endotransglycosylase in Escherichia coli. Journal of bacteriology 180, 3441-3447 (1998).

24. Paradis-Bleau, C. et al. Lipoprotein Cofactors Located in the Outer Membrane Activate Bacterial Cell Wall Polymerases. Cell 143, 1110-1120 (2010).

25. Typas, A. et al. Regulation of peptidoglycan synthesis by outer-membrane proteins. Cell 143, 1097-1109 (2010).

26. Paradis-Bleau, C., Kritikos, G., Orlova, K. \& Typas, A. A genome-wide screen for bacterial envelope biogenesis mutants identifies a novel factor involved in cell wall precursor metabolism. PloS Genet. 10, e1004056 (2014).

27. Schmidt, L. S., Botta, G. \& Park, J. T. Effects of furazlocillin, a beta-lactam antibiotic which binds selectively to PBP 3, on Escherichia coli mutants deficient in other penicillin-binding proteins. Journal of bacteriology 145, 632-637 (1981). 
28. Bernhardt, T. G. \& De Boer, P. A. J. Screening for synthetic lethal mutants in Escherichia coli and identification of EnvC (YibP) as a periplasmic septal ring factor with murein hydrolase activity. Mol. Microbiol. 52, 1255-1269 (2004).

29. Bernhardt, T. \& DeBoer, P. SlmA, a Nucleoid-Associated, FtsZ Binding Protein Required for Blocking Septal Ring Assembly over Chromosomes in. Mol. Cell 18, 555564 (2005).

30. Guzman, L. M., Belin, D., Carson, M. J. \& Beckwith, J. Tight regulation, modulation, and high-level expression by vectors containing the arabinose PBAD promoter. Journal of bacteriology 177, 4121-4130 (1995).

31. Babu, M. et al. Genetic Interaction Maps in Escherichia coli Reveal Functional Crosstalk among Cell Envelope Biogenesis Pathways. PLoS Genet. 7, e1002377 (2011).

32. Nichols, R. J., Sen, S., Choo, Y. J., Beltrao, P. \& Zietek, M. Phenotypic landscape of a bacterial cell. Cell 144, 143-156 (2011).

33. Uehara, T., Dinh, T. \& Bernhardt, T. G. LytM-Domain Factors Are Required for Daughter Cell Separation and Rapid Ampicillin-Induced Lysis in Escherichia coli. Journal of bacteriology 191, 5094-5107 (2009).

34. Finn, R. D., Bateman, A., Clements, J. \& Coggill, P. Pfam: the protein families database. Nucleic Acids Res. 32, D138-D141 (2013).

35. Holm, L. \& Rosenström, P. Dali server: conservation mapping in 3D. Nucleic Acids Res. 38, W545-W549 (2010).

36. Jing, X., Robinson, H. R., Heffron, J. D., Popham, D. L. \& Schubot, F. D. The catalytic domain of the germination-specific lytic transglycosylase SleB from Bacillus anthracisdisplays a unique active site topology. Proteins 80, 2469-2475 (2012).

37. Li, Y., Jin, K., Setlow, B., Setlow, P. \& Hao, B. Crystal Structure of the Catalytic Domain of the Bacillus cereus SleB Protein, Important in Cortex Peptidoglycan Degradation during Spore Germination. Journal of bacteriology 194, 4537-4545 (2012).

38. Giebel, J. D., Carr, K. A., Anderson, E. C. \& Hanna, P. C. The germination-specific lytic enzymes SleB, CwlJ1, and CwlJ2 each contribute to Bacillus anthracis spore germination and virulence. Journal of bacteriology 191, 5569-5576 (2009).

39. Heffron, J. D., Lambert, E. A., Sherry, N. \& Popham, D. L. Contributions of Four Cortex Lytic Enzymes to Germination of Bacillus anthracis Spores. Journal of bacteriology 192, 763-770 (2010).

40. Heffron, J. D., Sherry, N. \& Popham, D. L. In Vitro Studies of Peptidoglycan Binding and Hydrolysis by the Bacillus anthracis Germination-Specific Lytic Enzyme SleB. Journal of bacteriology 193, 125-131 (2010). 
41. Ashkenazy, H., Erez, E., Martz, E., Pupko, T. \& Ben-Tal, N. ConSurf 2010: calculating evolutionary conservation in sequence and structure of proteins and nucleic acids.

Nucleic Acids Res. 38, W529-33 (2010).

42. Zhou, R., Chen, S. \& Recsei, P. A dye release assay for determination of lysostaphin activity. Anal. Biochem. (1988).

43. Höltje, J. V., Mirelman, D., Sharon, N. \& Schwarz, U. Novel type of murein transglycosylase in Escherichia coli. Journal of bacteriology 124, 1067-1076 (1975).

44. Engel, H., Kazemier, B. \& Keck, W. Murein-metabolizing enzymes from Escherichia coli: sequence analysis and controlled overexpression of the slt gene, which encodes the soluble lytic transglycosylase. Journal of bacteriology 173, 6773-6782 (1991).

45. Lee, M. et al. Reactions of All Escherichia coliLytic Transglycosylases with Bacterial Cell Wall. Journal of the American Chemical Society 135, 3311-3314 (2013).

46. Ciccarelli, F. D. Toward Automatic Reconstruction of a Highly Resolved Tree of Life. Science 311, 1283-1287 (2006).

47. Letunic, I. \& Bork, P. Interactive Tree Of Life v2: online annotation and display of phylogenetic trees made easy. Nucleic Acids Res. 39, W475-8 (2011).

48. Krogh, A., Larsson, B. È. \& Heijne, von, G. Predicting transmembrane protein topology with a hidden Markov model: application to complete genomes. Journal of molecular biology 305, 567-580 (2001).

49. Kall, L., Krogh, A. \& Sonnhammer, E. L. L. Advantages of combined transmembrane topology and signal peptide prediction--the Phobius web server. Nucleic Acids Res. 35, W429-W432 (2007).

50. Lewenza, S., Vidal-Ingigliardi, D. \& Pugsley, A. P. Direct Visualization of Red Fluorescent Lipoproteins Indicates Conservation of the Membrane Sorting Rules in the Family Enterobacteriaceae. Journal of bacteriology 188, 3516-3524 (2006).

51. Zhu, Q. \& Casey, J. R. Topology of transmembrane proteins by scanning cysteine accessibility mutagenesis methodology. Methods 41, 439-450 (2007).

52. Pang, T., Savva, C. G., Fleming, K. G., Struck, D. K. \& Young, R. Structure of the lethal phage pinhole. Proceedings of the National Academy of Sciences 106, 18966-18971 (2009).

53. Harz, H., Burgdorf, K. \& Höltje, J.-V. Isolation and separation of the glycan strands from murein of Escherichia coli by reversed-phase high-performance liquid chromatography. Anal. Biochem. 190, 120-128 (1990). 
54. Karimova, G., Pidoux, J., Ullmann, A. \& Ladant, D. A bacterial two-hybrid system based on a reconstituted signal transduction pathway. Proceedings of the National Academy of Sciences 95, 5752-5756 (1998).

55. Uehara, T. \& Park, J. T. Growth of Escherichia coli: Significance of Peptidoglycan Degradation during Elongation and Septation. Journal of bacteriology 190, 3914-3922 (2008).

56. Curtis, N. A., Orr, D. \& Ross, G. W. Affinities of penicillins and cephalosporins for the penicillin-binding proteins of Escherichia coli K-12 and their antibacterial activity. Antimicrobial agents and chemotherapy, 16, 533-539 (1979).

57. Young, K. D. The Selective Value of Bacterial Shape. Microbiology and Molecular Biology Reviews 70, 660-703 (2006).

58. Glauner, B., Höltje, J. V. \& Schwarz, U. The composition of the murein of Escherichia coli. J. Biol. Chem. 263, 10088-10095 (1988).

59. Vollmer, W., Blanot, D. \& de Pedro, M. A. Peptidoglycan structure and architecture. FEMS microbiology reviews 32, 149-167 (2008).

60. Furchtgott, L., Wingreen, N. S. \& Huang, K. C. Mechanisms for maintaining cell shape in rod-shaped Gram-negative bacteria. Mol. Microbiol. 81, 340-353 (2011).

61. Wang, T.-S. A., Manning, S. A., Walker, S. \& Kahne, D. Isolated Peptidoglycan Glycosyltransferases from Different Organisms Produce Different Glycan Chain Lengths. Journal of the American Chemical Society 130, 14068-14069 (2008).

62. Perlstein, D. L., Zhang, Y., Wang, T.-S., Kahne, D. E. \& Walker, S. The Direction of Glycan Chain Elongation by Peptidoglycan Glycosyltransferases. Journal of the American Chemical Society 129, 12674-12675 (2007).

63. Buist, G., Steen, A., Kok, J. \& Kuipers, O. P. LysM, a widely distributed protein motif for binding to (peptido)glycans. Mol. Microbiol. 68, 838-847 (2008).

64. Kelley, L. A., Mezulis, S., Yates, C. M., Wass, M. N. \& Sternberg, M. J. E. The Phyre2 web portal for protein modeling, prediction and analysis. Nat Protoc 10, 845-858 (2015).

65. Bateman, A. \& Bycroft, M. The structure of a LysM domain from E. coli membranebound lytic murein transglycosylase D (MltD). Journal of molecular biology 299, 1113 1119 (2000).

66. Salama, N. R., Shepherd, B. \& Falkow, S. Global transposon mutagenesis and essential gene analysis of Helicobacter pylori. Journal of bacteriology 186, 7926-7935 (2004).

67. Zhang, H., Gao, S., Lercher, M. J., Hu, S. \& Chen, W. H. EvolView, an online tool for visualizing, annotating and managing phylogenetic trees. Nucleic Acids Res. 40, W569W572 (2012). 
68. Banzhaf, M. et al. Cooperativity of peptidoglycan synthases active in bacterial cell elongation. Mol. Microbiol. 85, 179-194 (2012).

69. Tsui, H.-C. T. et al. Suppression of a Deletion Mutation in the Gene Encoding Essential PBP2b Reveals a New Lytic Transglycosylase Involved in Peripheral Peptidoglycan Synthesis in Streptococcus pneumoniae D39. Mol. Microbiol. (2016).

70. Miller, J. H. Experiments in molecular genetics. (1972).

71. Guyer, M. S., Reed, R. R. \& Steitz, J. A. Identification of a sex-factor-affinity site in E. coli as $\gamma \delta$. Cold Spring Harbor symposia on quantitative biology 45, (1981).

72. Yu, D., Ellis, H. M., Lee, E. C. \& Jenkins, N. A. An efficient recombination system for chromosome engineering in Escherichia coli. Proceedings of the National Academy of Sciences 97, 5978-5983 (2000).

73. Datsenko, K. A. \& Wanner, B. L. One-step inactivation of chromosomal genes in Escherichia coli K-12 using PCR products. Proceedings of the National Academy of Sciences 97, 6640-6645 (2000).

74. Uehara, T., Parzych, K. R., Dinh, T. \& Bernhardt, T. G. Daughter cell separation is controlled by cytokinetic ring-activated cell wall hydrolysis. The EMBO journal 29, 1412-1422 (2010).

75. Bendezú, F. O., Hale, C. A., Bernhardt, T. G. \& De Boer, P. A. J. RodZ (YfgA) is required for proper assembly of the MreB actin cytoskeleton and cell shape in E. coli. The EMBO journal 28, 193-204 (2008).

76. Morlot, C., Uehara, T., Marquis, K. A., Bernhardt, T. G. \& Rudner, D. Z. A highly coordinated cell wall degradation machine governs spore morphogenesis in Bacillus subtilis. Genes \& development 24, 411-422 (2010).

77. Hale, C. A. \& de Boer, P. A. Recruitment of ZipA to the septal ring of Escherichia coli is dependent on FtsZ and independent of FtsA. Journal of bacteriology 181, 167-176 (1999).

78. Baba, T. et al. Construction of Escherichia coli K-12 in-frame, single-gene knockout mutants: the Keio collection. Mol Syst Biol 2, (2006).

79. Johnson, J. E., Lackner, L. L., Hale, C. A. \& De Boer, P. A. J. ZipA is required for targeting of DMinC/DicB, but not DMinC/MinD, complexes to septal ring assemblies in Escherichia coli. Journal of bacteriology 186, 2418-2429 (2004).

80. Haldimann, A. \& Wanner, B. L. Conditional-Replication, Integration, Excision, and Retrieval Plasmid-Host Systems for Gene Structure-Function Studies of Bacteria. Journal of bacteriology 183, 6384-6393 (2001). 
81. de Boer, P., Crossley, R. E. \& Rothfield, L. I. A division inhibitor and a topological specificity factor coded for by the minicell locus determine proper placement of the division septum in E. coli. Cell 56, 641-649 (1989).

82. Peters, N. T., Dinh, T. \& Bernhardt, T. G. A Fail-Safe Mechanism in the Septal Ring Assembly Pathway Generated by the Sequential Recruitment of Cell Separation Amidases and Their Activators. Journal of bacteriology 193, 4973-4983 (2011). 
Chapter 3. Discussion 


\subsection{SUMMARY OF RESULTS}

Lytic transglycosylases are crucial for PG metabolism in most bacteria. Although it has been challenging to unravel the precise physiological role of many LTs, they have been implicated in a range of functions including cell wall expansion, septation, insertion of transenvelope complexes, and PG recycling and turnover ${ }^{1}$. Additionally, but perhaps counterintuitively, LTs may also act in the early stages of nascent PG polymerization. In most bacteria,

the glycan chains of PG are capped with an anhydro-muropeptide ${ }^{2,3}$. Pulse-labelling experiments have shown that this anhydro cap is introduced shortly after or concomitantly with termination of polymerization ${ }^{4,5}$. Additionally, the mechanism by which PG polymerizing enzymes terminate glycan strand synthesis is unclear ${ }^{6}$. For this reason, lytic transglycosylases have long been believed to be involved in the termination of nascent PG polymerization, but a lytic transglycosylase responsible for this activity has yet to be identified. Here, we have identified MltG, the founding member of a new family of lytic transglycosylases. In E. coli, we have shown that MltG is an endo-acting lytic transglycosylase well-poised in the inner membrane to act as a potential terminase for PG polymerization.

\subsection{ROLE OF MLTG IN CELL WALL ELONGATION}

Lytic transglycosylases with MltG-like domains are distributed throughout gram-negative and gram-positive bacteria. Notably, a major fraction of species lacking MltG homologs are either coccal species or bacteria that lack cell walls, such as Mycoplasma. In contrast, almost all rod-shaped and ovococcal bacteria assessed encode MltG-like proteins. Rod-shaped and ovococcal bacteria are believed to possess two independent PG synthesis complexes, one involved in inserting PG along the sidewall to elongate the cell and a second for generating a septum during cell division. The observed pattern of MltG conservation implies that MltG 
function may be particularly important to promote proper cell elongation.

Consistent with this idea, recent work from Winkler and colleagues demonstrates that $\mathrm{MltG}$ is an essential component of the elongation (peripheral synthesis) machinery in the grampositive species, Streptococcus pneumoniae $\left({ }^{S p} \mathrm{MltG}\right){ }^{7}$. Cells lacking ${ }^{S p} m l t G$ round up into spheres and are ultimately inviable without compensatory suppressor mutations ${ }^{7}$. Similar to $\mathrm{MltG}$ in E. coli $\left({ }^{E c} \mathrm{MltG}\right),{ }^{S p} \mathrm{MltG}$ localizes to the cytoplasmic membrane and has a large extracellular, "MltG-like" domain. However, unlike its E. coli counterpart, it additionally possesses a large cytoplasmic domain of unknown function. Although in vitro activity could not be demonstrated with ${ }^{S p} \mathrm{MltG}$, its periplasmic domain shares a high degree of sequence homology with MltG from E. coli, including a glutamate (E428) in ${ }^{S p} \mathrm{MltG}$ that overlaps with the catalytic glutamate of ${ }^{E c} \mathrm{MltG}$. In support of its conserved function as a lytic transglycosylase, pneumococcal strains encoding a variant with a substitution at this predicted catalytic residue ${ }^{S p} \mathrm{MltG}$ (E428Q) phenocopy $\Delta m l t G^{7}$. Further, this phenotype could be complemented with expression of either $\mathrm{WT}^{S p} \mathrm{MltG}$ or ${ }^{E c} \mathrm{MltG}$, suggesting that $\mathrm{MltG}$ performs similar roles in vivo in both gram-positive pneumococcus and gram-negative E. coli ${ }^{7}$.

An extensive web of genetic interactions has been observed in relation to $m l t G$ in $S$. pneumoniae providing valuable insights into the relationship between ${ }^{S p} \mathrm{MltG}$ and other essential cell wall elongation factors ${ }^{7}$. Similar to E. coli, cell wall elongation in pneumococcus depends on a complex of proteins including PBPs, in this case PBP1a, a class A bifunctional TP/GT with homology to PBP1b in E. coli, and PBP2b, a class B monofunctional $\mathrm{TP}^{8}$. Also in this complex are a host of integral membrane proteins with less defined functions, including MreC, MreD, RodA, and RodZ. With exception of PBP1a, all components of this complex are essential for viability $^{8}$. 
Work on pneumococcal MltG suggests there is a specific coupling between MltG and the class A PBP, PBP1a. Interestingly, the essentiality of ${ }^{S p} m l t G$ is suppressed by inactivation of PBP1a, indicating that PBP1a activity is detrimental to cells when ${ }^{S p} \mathrm{MltG}$ is absent ${ }^{7}$. One possible reason for this is that without the opposing cleavage of ${ }^{\mathrm{Sp}} \mathrm{MltG}, \mathrm{PBP} 1 \mathrm{a}$ may polymerize aberrant or excessively long glycan strands, leading to a toxic build up of uncrosslinked glycan chains. Deletion of ${ }^{S p} m l t G$ specifically selects for suppressor mutations in PBP1a but not other PG synthases indicating that the activity of ${ }^{\mathrm{Sp}} \mathrm{MltG}$ is preferentially associated with $\mathrm{PG}$ synthesis by PBP1a ${ }^{7}$. The precise nature of the relationship between PBP1a and ${ }^{\mathrm{Sp}} \mathrm{MltG}$ and whether they directly interact is unknown.

Additional genetic and co-localization evidence reveals a relationship between PBP 1a/ ${ }^{\mathrm{Sp}} \mathrm{MltG}$ and two factors essential for elongation, MreCD and RodZ ${ }^{9,7}$. MreCD and RodZ are not cell wall modifying enzymes. Instead, they may serve a regulatory function by modulating the activity or localization of PBP1a and/or MltG ${ }^{9,7}$. Consistent with this, in both $E$. coli and S. pneumoniae, MreC is dispensable if MltG is inactivated, supporting the hypothesis that $\mathrm{MreC}$ is required for proper $\mathrm{MltG}$ regulation or localization ${ }^{9,7}(P$. Rohs, Personal Communication). Accordingly, previous work in Caulobacter crescentus has shown that MreC is necessary for the spatial organization of a different lytic transglycosylase, MltA ${ }^{10}$. Additional studies are required to further elucidate the relationship between $\mathrm{MreC}, \mathrm{MreD}$, or RodZ and cell wall modifying enzymes involved in elongation. Altogether, the relationships observed between MltG and peripheral PG synthesis factors in pneumococcus are consistent with our original model that MltG works closely with PG synthases during cell wall expansion. Further, these results support the idea that the role of MltG in cell wall elongation may be broadly conserved across gram-negative and gram-positive bacteria. 


\subsection{FUTURE DIRECTIONS}

How do bacteria coordinate synthesis and hydrolysis?

Cell wall biogenesis is a balancing act achieved through the careful regulation of PG construction and break down. Imbalances in either direction can be harmful to the cell. Excessive degradation relative to synthesis can be lethal as observed with the toxicity of MltG overexpression in E. coli strains lacking PBP1b. In the other direction, PG synthesis can become toxic when MltG is lost, as demonstrated with PBP1a in pneumococcus ${ }^{7}$, although a similar relationship has not been detected in E. coli. In general, it is not known how bacteria maintain coordination between lytic transglycosylases and PG synthases .

One way balance could be achieved is through integration of PG synthases and LTs in multi-enzyme complexes, as previously proposed. The bacterial two hybrid data in E. coli and genetic and co-localization evidence in pneumococcus indeed appears to place MltG in the context of other key players required for cell wall elongation. In this situation, it could be envisioned that components of the complex not only co-localize but also directly modulate one another to achieve balance. However, given the intricate regulatory strategies that have previously been demonstrated for cell wall hydrolases (See Chapter 1), the picture is likely much more complex than this. Coordination is presumably achieved via multiple layers of regulation, which may or may not involve complex formation.

Based on bacterial two hybrid assays, we have shown that MltG works in proximity to PBP1b, one of the main PG synthases in E. coli. We also have obtained genetic evidence linking MltG with MreC as well as PBP1b and LpoB, an activator of PBP1b(P. Rohs and J. Bohrhunter, personal communication). This leads us to hypothesize that LpoB, PBP1b, and MltG and/or MreC work together to coordinate PG synthesis and hydrolysis. Surprisingly, however, we can 
not detect direct protein-protein interactions between MltG and PBP1b or LpoB in-vitro (unpublished). It is possible that MltG may interact with LpoB and/or PBP1b via intermediary factors similar to MipA, a protein that bridges an interaction between MltA and PBP1b ${ }^{11}$. Alternatively, MltG recruitment to sites of nascent PG synthesis could occur merely through its affinity for uncrosslinked, nascent glycan material rather than through protein-protein associations. The idea that MltG works in complex is particularly brought into question by the finding that ${ }^{\mathrm{Ec}} \mathrm{MltG}$ from E. coli can substitute for ${ }^{\mathrm{Sp}} \mathrm{MltG}$ in $S$. pneumoniae despite the distant relationship between the two organisms. MltG from E. coli is unlikely to have the same proteinprotein interactions in such a vastly different organism, suggesting that MltG may be recruited to sites of synthesis by other means, such as substrate affinity.

Co-immunoprecipitation experiments with MltG will help identify other protein-protein interactions, and provide further insight into cell wall factors that may be associated with MltG function or regulation. It will also be interesting to examine whether the association of PBP1b and MltG observed in bacterial two-hybrid assays is dependent on the glycosyltransferase activity of PBP1b, which would help elucidate the role of substrate affinity in MltG recruitment. Additionally, co-localization studies may provide insight into the association and movement of MltG relative to $\mathrm{PG}$ synthesizing complexes.

How is MltG activity regulated in E. coli?

Mechanisms must be in place to control MltG activity to ensure that it cuts the cell wall at the right time and place. To probe for potential regulatory mechanisms, we can use genetic selections to i) identify potential regulatory domains within MltG and ii) identify additional factors that may be important for regulating MltG activity. Similar to other cell hydrolases 
studied in our lab, MltG may be maintained in an "off" state in vivo until its activity is required, at which point another mechanism may turn it "on". Indeed, MltG displays very low levels of catalytic activity in vitro suggesting that it may require activation by other factors for full activity. Unmodulated hydrolytic activity caused by mis-regulation would lead to excessive degradation of the cell wall in vivo and cause cell lysis. Consequently, domains or residues important for MltG activity can be identified by searching for hyper-lytic variants of MltG that are no longer properly regulated. To identify such variants, a plasmid release enrichment strategy can be used to isolate hyperactive $m l t G$ mutants. This strategy was previously optimized in our lab and used to uncover the auto-regulatory domain of other $E$. coli cell wall hydrolases ${ }^{12}$. Hyper-lytic MltG variants can also serve as a useful tool to search for mutations elsewhere in the genome that suppress this toxicity. Suppressors of hyper-lytic MltG may occur in genes encoding factors that regulate MltG activity, localization, complex formation, or expression. Additionally, to identify potential regulators, we can also use a biochemical approach and examine how proteins that directly interact with MltG modulate MltG activity in-vitro.

\section{Do other lytic transglycosylases work redundantly with MltG?}

MltG is known to be essential in some bacteria ${ }^{13}$, but it is not required for normal growth in E. coli. Given the extensive number of lytic transglycosylases encoded by E. coli, it would not be surprising if other LTs, either known or unknown, can substitute for MltG in its absence. Additionally, evidence thus far appears to link MltG specifically to PG synthesis complexes involved in cell wall elongation. It is not clear whether MltG or an analogous LT is required for termination of synthesis during septum formation. A synthetic lethal screening approach, such as TnSeq, will allow us to systematically probe for genes that lie in a parallel pathway with $m l t G$. 
One caveat of this approach is that functional redundancy among the extensive number of LTs in E. coli may obscure pathways that might otherwise be synthetically lethal with $\Delta m l t G$. To overcome this, TnSeq can also be performed on a range of double and triple LT mutants containing $\Delta m l t G$. Additionally, although previous studies have examined LT deletion mutants lacking up to six LTs, multiple LT deletion mutants also lacking MltG have yet to be examined. Systematically assessing how multiple LT deletions, including MltG, influence growth, morphology, and cell wall composition may help us uncover essential sets of LTs associated with particular cell wall processes such as division, cell wall elongation, or PG turnover.

\subsection{THE POTENTIAL FOR LTS AS DRUG TARGETS}

As established in the past several chapters, lytic transglycosylases are critical for proper cell wall physiology in most bacteria. Due to the essential and unique nature of the cell wall, it remains an attractive target for antibacterial treatments. Unfortunately, extensive use of $\beta$ lactams since their first clinical application in the mid-1900s has accelerated the emergence of resistant bacterial pathogens ${ }^{14}$. Lytic transglycosylases have yet to be clinically utilized as antibiotic targets, but given their unique specificity and active site topology relative to PBPs, they provide an opportunity to search for antibiotics distinct from B-lactams.

LTs are additionally an appealing avenue of study for the discovery of antibiotics that work in combination with $\beta$-lactams. Treatment involving combination therapies can be clinically effective, as demonstrated by regimens that combine amoxicillin, a $\beta$-lactam antibiotic, with clavulanic acid, an inhibitor of $\beta$-lactamase ${ }^{15}$. An alternative but complementary approach is to suppress induction of $\beta$-lactamase production during $\beta$-lactam treatment. $\beta$-lactamase induction is intimately tied to the activity of LTs in certain gram-negative bacteria ${ }^{16}$. In these 
cases, deletion or chemical inhibition of LTs can prevent induction of ß-lactamases and enhance susceptibility to $\beta$-lactam antibiotics ${ }^{17-20}$. Bulgecin is one example of a known chemical inhibitor of lytic transglycosylases. Bulgecin alone does not impair growth or kill bacteria, but it shows strong synergism with $\beta$-lactam antibiotics ${ }^{17-20}$. Overall, combined treatment with $\beta$-lactam and inhibiters of lytic transglycosylases may represent an alternative strategy to extend the utility of our current arsenal of $\beta$-lactam antibiotics.

Lytic transglycosylases are also attractive targets due to their direct contributions to bacterial pathogenicity in certain instances. For example, inhibition of LTs may attenuate Neisseria gonorrhoeae pathogenicity by preventing release of cytotoxic anhydro-muropeptides, which are responsible for many of the symptoms associated with infection ${ }^{21}$. Additionally, LTs may be important targets as accessory factors in secretion systems required for virulence ${ }^{22}$. For example, Helicobacter pylori is rendered avirulent by mutation of a putative LT, HP0523, essential for type IV secretion ${ }^{23}$. Gven the diversity of LT active sites, drugs developed to target specialized LTs in specific organisms may be relatively narrow-spectrum.

In the case of MltG, it is not clear how its inhibition would influence $\beta$-lactam resistance or pathogenicity. To assess this, compounds capable of inhibiting MltG will first need to be identified. Interestingly, MltG homologs are reported as essential in pathogenic organisms such as Helicobacter pylori and Mycobacterium tuberculosis ${ }^{13,24}$, making it an appealing target. However, one important caveat to consider is that in the case of S. pneumoniae, MltG essentiality is readily bypassed by suppresser mutations in $\mathrm{PBP} \mathrm{a}^{7}$, although it is not known how these mutations impact virulence. It remains to be explored how deletion of MltG influences bacterial pathogenicity and whether it could be a useful target in single or combined treatments. 


\subsection{CONCLUSION}

Work in this thesis has uncovered a unique family of lytic transglycosylases that is broadly conserved across gram-negative and gram-positive species. After our initial biochemical characterization of MltG (Chapter 2), additional genetic evidence has come to light linking MltG to key components of the cell wall elongation machinery in E. coli and S. pneumococcus (Chapter 3). Altogether, this evidence is consistent with our model that MltG fulfills the longproposed role of a PG "terminase" involved in releasing nascent glycan chains from the PG synthesis machinery and capping the chains with a 1,6-anhydro-MurNAc residue ${ }^{25}$.

Active termination of glycan strand polymerization may be important for PG biogenesis on several levels. For example, termination of PG synthesis may be required to prevent the toxic accumulation of uncrosslinked glycan chains in regions of the cell wall where cross-linking sites have been saturated. As discussed in Chapter 1, another LT (Slt) has been proposed to serve a quality control function by degrading uncrosslinked glycan chains that accumulate during antibiotic treatment ${ }^{26}$. One hypothesis is that MltG may act upstream of Slt to proactively halt synthesis before glycan strands can accumulate to toxic levels. Deletion of ${ }^{S p} m l t G$ in pneumococcus indeed appears to render synthesis by PBP1a toxic ${ }^{7}$, supporting the idea that MltG may act as a quality control factor. Active termination of PG polymerization may also be important for modulating the length of glycan strands in the cell wall matrix. Little is known about what factors influence glycan chain length in the cell wall and what role glycan length plays in determining the final shape and size of the cell. By identifying factors that influence glycan strand length, we can begin to probe for how changes in glycan strand length influence bacterial morphogenesis. Ultimately, further characterization of MltG will provide important 
insights into the intricate process of PG biogenesis, aiding us in the search for new vulnerabilities in cell wall metabolism amenable to antibiotic targeting. 


\subsection{REFERENCES}

1. Scheurwater, E., Reid, C. W. \& Clarke, A. J. Lytic transglycosylases: Bacterial spacemaking autolysins. The International J. of biochem. \& cell biology 40, 586-591 (2008).

2. Höltje, J. V., Mirelman, D., Sharon, N. \& Schwarz, U. Novel type of murein transglycosylase in Escherichia coli. Journal of bacteriology 124, 1067-1076 (1975).

3. Hartmann, R., Höltje, J. V. \& Schwarz, U. Targets of penicillin action in Escherichia coli. Nature 235, 426-429 (1972).

4. Glauner, B. \& al, E. Growth pattern of the murein sacculus of Escherichia coli. J. Biol. Chem. 265, 18988-18996 (1990).

5. Burman, L. G. \& Park, J. T. Changes in the composition of Escherichia coli murein as it ages during exponential growth. Journal of bacteriology (1983).

6. van Heijenoort, J. Peptidoglycan Hydrolases of Escherichia coli. Microbiology and Molecular Biology Reviews 75, 636-663 (2011).

7. Tsui, H.-C. T. et al. Suppression of a Deletion Mutation in the Gene Encoding Essential PBP2b Reveals a New Lytic Transglycosylase Involved in Peripheral Peptidoglycan Synthesis in Streptococcus pneumoniae D39. Mol. Microbiol. (2016).

8. Sham, L.-T., Tsui, H.-C. T., Land, A. D., Barendt, S. M. \& Winkler, M. E. Recent advances in pneumococcal peptidoglycan biosynthesis suggest new vaccine and antimicrobial targets. Current opinion in microbiology 15, 194-203 (2012).

9. Land, A. D. \& Winkler, M. E. The Requirement for Pneumococcal MreC and MreD Is Relieved by Inactivation of the Gene Encoding PBP1a. Journal of bacteriology 193, 4166-4179 (2011).

10. Divakaruni, A. V., Baida, C., White, C. L. \& Gober, J. W. The cell shape proteins MreB and MreC control cell morphogenesis by positioning cell wall synthetic complexes. Mol. Microbiol. 66, 174-188 (2007).

11. Vollmer, W., Rechenberg, von, M. \& Höltje, J. V. Demonstration of Molecular Interactions between the Murein Polymerase PBP1B, the Lytic Transglycosylase MltA, and the Scaffolding Protein MipA of Escherichia coli. Journal of Biological Chemistry 274, 6726-6734 (1999).

12. Yang, D. C., Tan, K., Joachimiak, A. \& Bernhardt, T. G. A conformational switch controls cell wall-remodelling enzymes required for bacterial cell division. Mol. Microbiol. 85, 768-781 (2012).

13. Luo, H., Lin, Y., Gao, F., Zhang, C.-T. \& Zhang, R. DEG 10, an update of the database of essential genes that includes both protein-coding genes and noncoding genomic elements. Nucleic Acids Res. 42, D574-80 (2014). 
14. Jed F Fisher, Samy O Meroueh, A. \& Mobashery, S. Bacterial Resistance to $\beta$-Lactam Antibiotics: Compelling Opportunism, Compelling Opportunity $\dagger$. Chemical reviews (2005).

15. Brown, E. D. \& Wright, G. D. Antibacterial drug discovery in the resistance era. Nature 529, 336-343 (2016).

16. Fisher, J. F. \& Mobashery, S. The sentinel role of peptidoglycan recycling in the $\beta$-lactam resistance of the Gram-negative Enterobacteriaceae and Pseudomonas aeruginosa. Bioorg. Chem. 56, 41-48 (2014).

17. Imada, A., Kintaka, K., Nakao, M. \& Shinagawa, S. Bulgecin, a bacterial metabolite which in concert with beta-lactam antibiotics causes bulge formation. J. Antibiot. 35, 1400-1403 (1982).

18. Kraft, A. R., Prabhu, J., Ursinus, A. \& Höltje, J.-V. Interference with murein turnover has no effect on growth but reduces beta-lactamase induction in Escherichia coli. Journal of bacteriology 181, 7192-7198 (1999).

19. Heidrich, C., Ursinus, A., Berger, J., Schwarz, H. \& Höltje, J. V. Effects of Multiple Deletions of Murein Hydrolases on Viability, Septum Cleavage, and Sensitivity to Large Toxic Molecules in Escherichia coli. Journal of bacteriology 184, 6093-6099 (2002).

20. Korsak, D., Liebscher, S. \& Vollmer, W. Susceptibility to Antibiotics and -Lactamase Induction in Murein Hydrolase Mutants of Escherichia coli. Antimicrobial agents and chemotherapy 49, 1404-1409 (2005).

21. Chan, Y. A., Hackett, K. T. \& Dillard, J. P. The Lytic Transglycosylases of Neisseria gonorrhoeae. Microbial Drug Resistance 18, 271-279 (2012).

22. Baron, C. \& Coombes, B. Targeting bacterial secretion systems: benefits of disarmament in the microcosm. Infect Disord Drug Targets 7, 19-27 (2007).

23. Rohde, M., Püls, J., Buhrdorf, R., Fischer, W. \& Haas, R. A novel sheathed surface organelle of the Helicobacter pylori cag type IV secretion system. Mol. Microbiol. 49, 219-234 (2003).

24. Salama, N. R., Shepherd, B. \& Falkow, S. Global transposon mutagenesis and essential gene analysis of Helicobacter pylori. Journal of bacteriology (2004).

25. Kraft, A., Templin, M. \& 1tje, J. H. O. Membrane-bound lytic endotransglycosylase in Escherichia coli. Journal of bacteriology 180, 3441-3447 (1998).

26. Cho, H., Uehara, T. \& Bernhardt, T. G. Beta-lactam antibiotics induce a lethal malfunctioning of the bacterial cell wall synthesis machinery. Cell 159, 1300-1311 (2014). 